\title{
DEVELOPMENT OF CAL POLY'S SHOCK TABLE
}

\author{
A Thesis \\ presented to \\ the Faculty of California Polytechnic State University, \\ San Luis Obispo
}

\author{
In Partial Fulfillment \\ of the Requirements for the Degree \\ Master of Science in Aerospace Engineering
}

by

Christopher D. Risner

December 2016 
(C) 2016

Christopher D. Risner

ALL RIGHTS RESERVED 
TITLE: Development of Cal Poly's Shock Table

AUTHOR: Christopher D. Risner

DATE SUBMITTED: December 2016

COMMITTE CHAIR: Kira Abercromby, Ph. D.

Associate Professor of Aerospace Engineering

COMMITTEE MEMBER: David Esposto

Retired Lecturer - Cal Poly San Luis Obispo

Retired Chief Engineer - Boeing

COMMITTEE MEMBER: $\quad$ Eric Mehiel, Ph. D.

Professor of Aerospace Engineering

COMMITTEE MEMBER: David Pignatelli, M.S.

Aerospace Engineer, CubeSat Program

COMMITTEE MEMBER: Jordi Puig-Suari, Ph. D.

Professor of Aerospace Engineering 


\author{
ABSTRACT \\ Development of Cal Poly's Shock Table \\ Christopher D. Risner
}

Shock is one of the environmental tests that a spacecraft must pass before being cleared for launch. Shock testing poses a challenging data acquisition issue and careful selection of equipment is crucial to creating a successful shock test facility. Cal Poly's CubeSat programs can currently perform all environmental testing other than shock themselves, so a quality shock table would be useful. Previous groups of students had developed a shock table, and this paper details the improvement and characterization of that shock table's behavior. Several adjustable parameters were tested and documented to discover trends in the shock table's response to an impact from a pendulum hammer. Then a test meant to mimic an actual shock test was performed. The CubeSat program provided a component to be tested and a requirement to be met. The nominal requirement is proprietary and cannot be given here, and additional stipulations included the test data being within a given tolerance band and at least $50 \%$ of the test data having a larger magnitude than the nominal requirement. The requirement needed to be met in all three of the component's axes. The component was mounted to the shock table and acceleration data was collected and analyzed. A successful test was conducted in one axis, which was the result of impacting the large face of the aluminum shock table plate. The tests in the other two axes, conducted with impacts to the side of the aluminum plate, failed to meet the requirement. A finite element model of the table was developed and correlated to the test data. A new way of attaching the test component to the table was developed that would allow for testing in all three axes to be performed with impacts to the large face of the aluminum plate. A dynamic finite element analysis was performed, and the results indicate that this new attachment method should allow the requirement to be met in all three axes. The shock table is currently fully operational and can be used for testing and teaching purposes. With the implementation of the new attachment method, it is believed that the CubeSat program's requirements can be met as well. 


\section{ACKNOWLEDGMENTS}

Many people deserve credit and appreciation for helping me through both this thesis and my Cal Poly career.

Cody Thompson was invaluable with his machining skills, sage advice, friendly demeanor, and overall level of handiness. Whenever I needed something done that was beyond my skill set (frequently), Cody was always willing to lend a hand. If I couldn't find Cody, Kyle and Greg were usually around and had to deal with their fair share of me bugging them. They were always helpful, advised me when I was stuck with making something for my project, and often completed pieces for me themselves. Kendra Bubert is the glue that holds the Aero department together, and she helped me navigate the maze of paperwork that comes with the blended program. Arash Mehrparvar answered dozens of emails from me pestering him with questions about the mystery that is shock testing. He always answered and I'm grateful for his input. Also, the folks at Microstar Laboratories were incredibly helpful and patient with me as I got the data acquisition system up and running.

My friends and roommates were always good for a distraction (perhaps too good) whenever I was tired of working and wanted a break. My family has always been supportive, and they were especially important these last few months in encouraging me to buckle down and just finish the project.

I would like to thank Dr. P for agreeing to be on my committee without even having met me, and for answering my questions when we did meet. Dave Pignatelli agreed to be on my committee last minute and secured the Test-POD that we used for 
testing. He should be commended for sitting through days of testing with me while I struggled to meet the test requirements. Dave Esposto knows a lot about a lot, and shock testing was no different. He helped me understand how shock testing is usually done in industry and met with me several times to answer whatever questions I had. He also secured the funding for the new data acquisition system, without which this project wouldn't have been possible. I've been taking classes with Dr. Mehiel since sophomore year, and I worked on ADCS in design so obviously I had to have him on my committee. He helped me with a fundamental misunderstanding I had about data acquisition and processing digitized data, and he has always been helpful and patient with whatever questions I may have had. And of course, there's my advisor Dr. A. I think her classes probably accounted for half of my Cal Poly coursework and some of my favorite material. Luckily I never had to buy pizza or donuts for a class, but little things like that and big things like organizing extra time outside of class to observe a satellite trajectory that we had calculated as a project show the dedication she has to her students. I'm sure I speak for all of her past students in saying that we notice the extra effort, we appreciate it, and it does make a difference. 


\section{TABLE OF CONTENTS}

Page

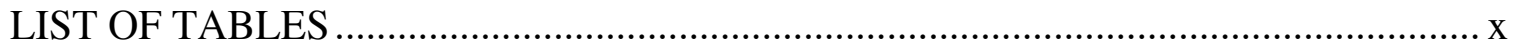

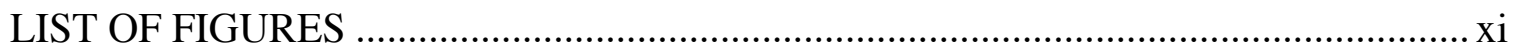

\section{CHAPTER}

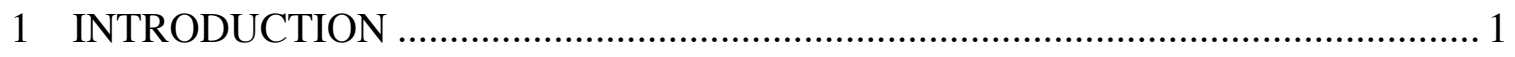

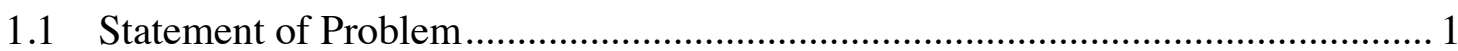

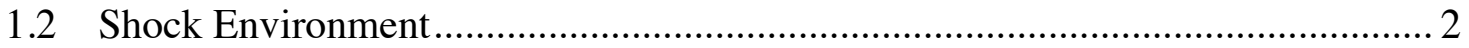

1.3 Shock Testing ...................................................................................... 4

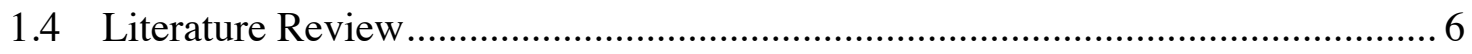

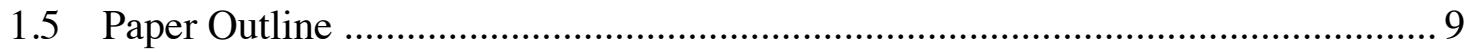

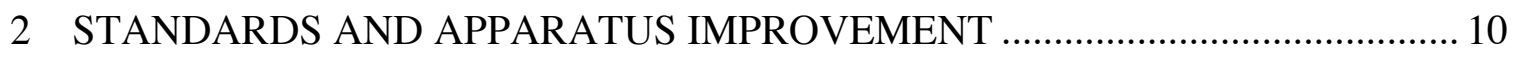

2.1 Data Acquisition Standards and Design ....................................................... 10

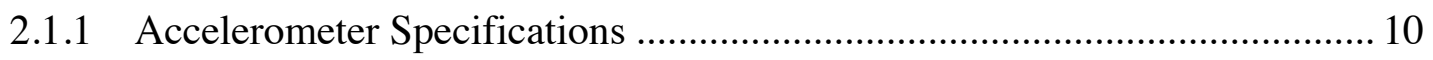

2.1.2 Signal Conditioner Specifications ...................................................... 12

2.1.3 Analog Anti-Aliasing Filter Specifications ......................................... 12

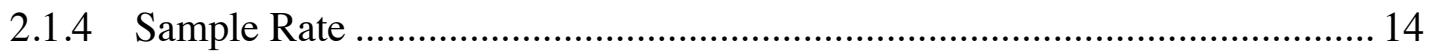

2.1.5 Selected Data Acquisition Equipment ..................................................... 15

2.2 Analysis Standards and Design............................................................. 16

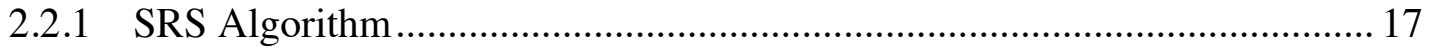

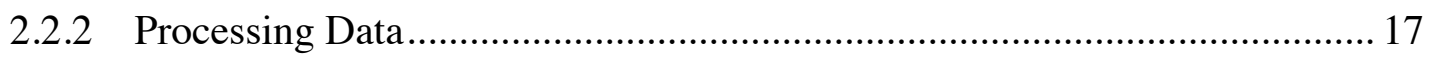

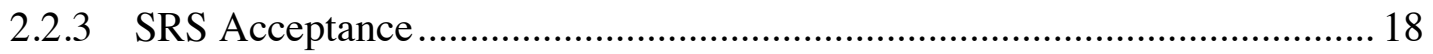

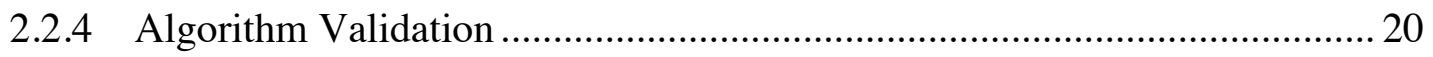


2.3 Apparatus Design .............................................................................. 21

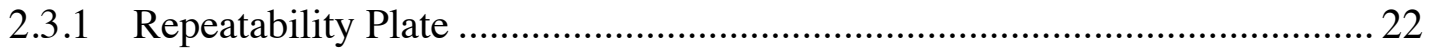

2.3.2 Floor Anchors ................................................................................. 23

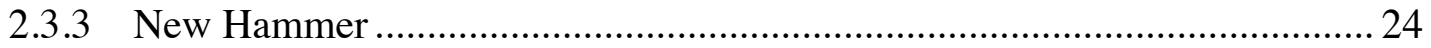

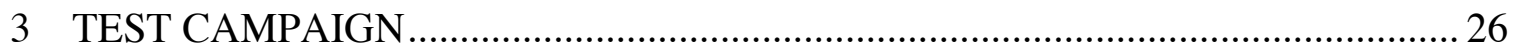

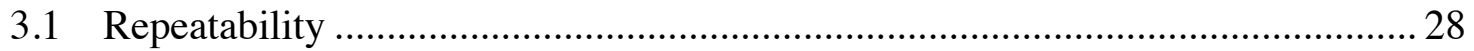

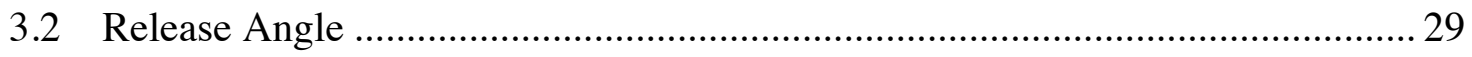

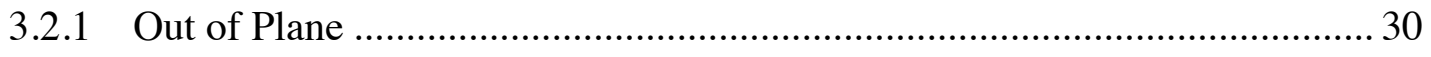

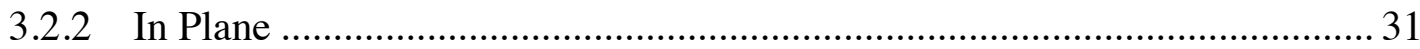

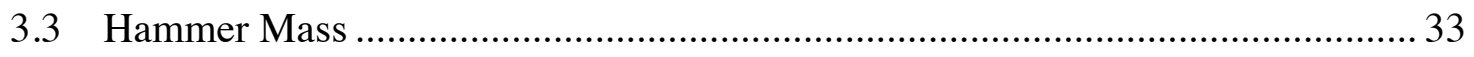

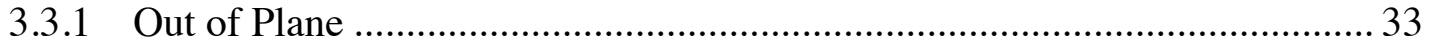

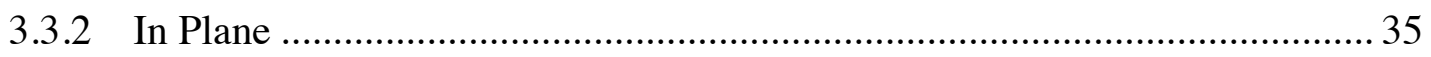

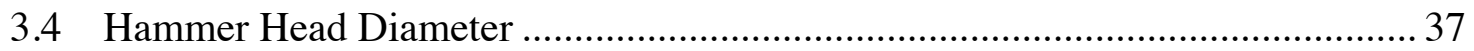

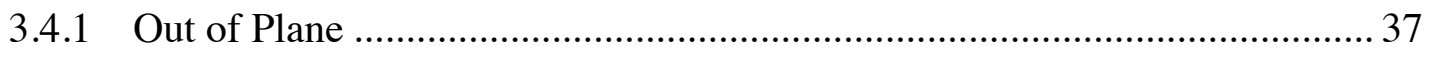

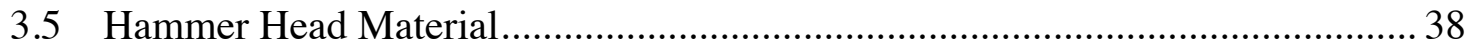

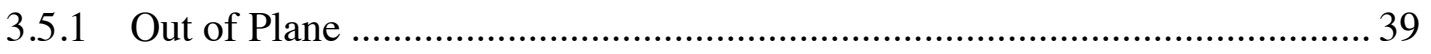

3.6 Mechanical Filter at Impact .................................................................... 40

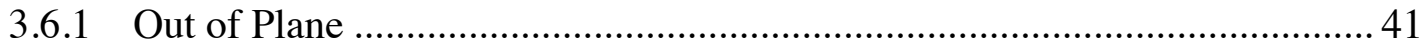

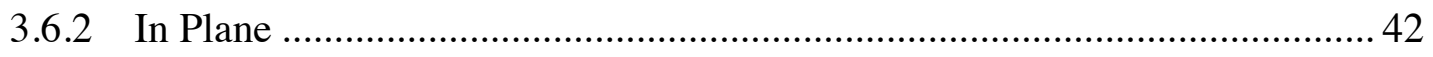

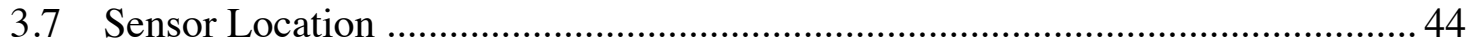

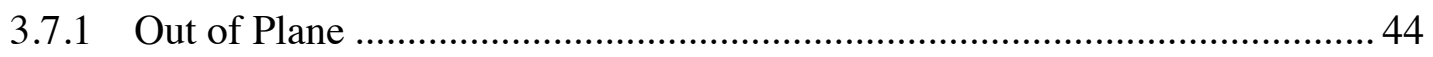

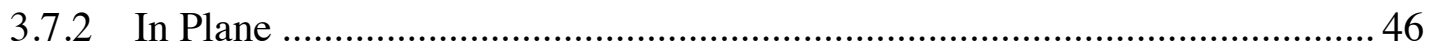

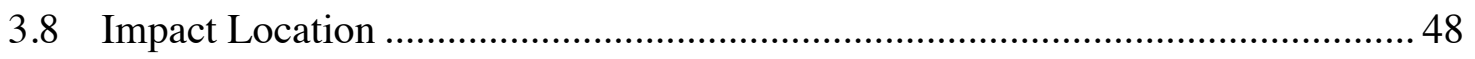




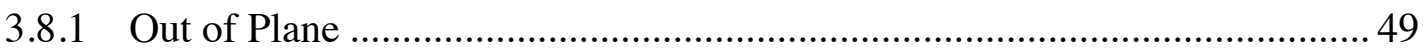

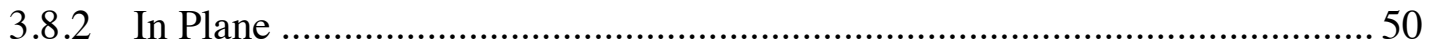

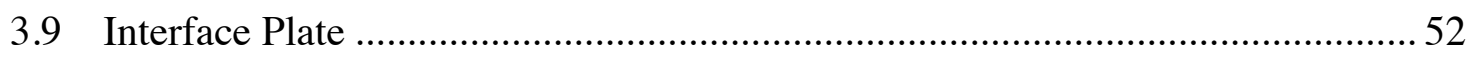

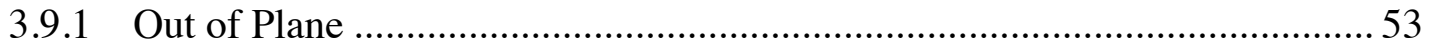

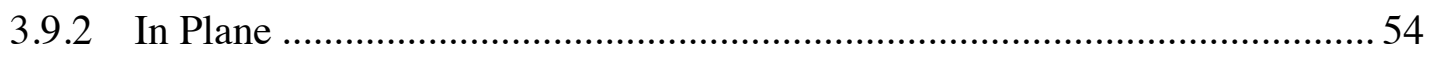

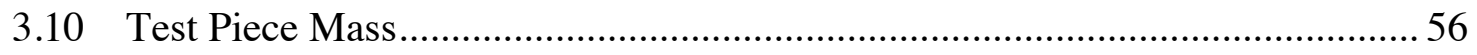

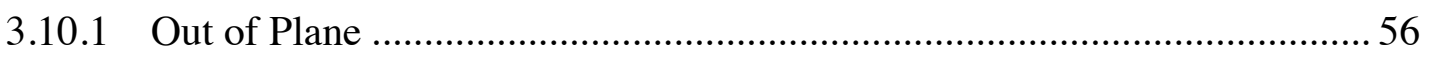

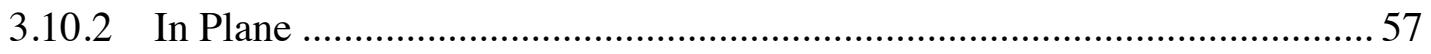

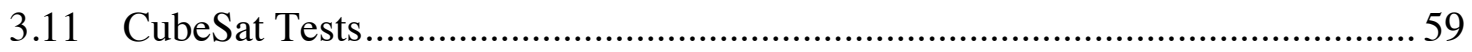

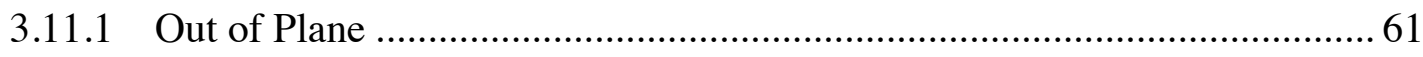

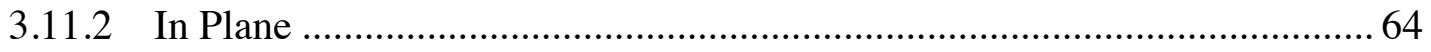

4 FINITE ELEMENT MODEL AND ANALYSIS ……………………………........... 67

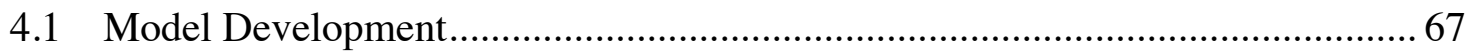

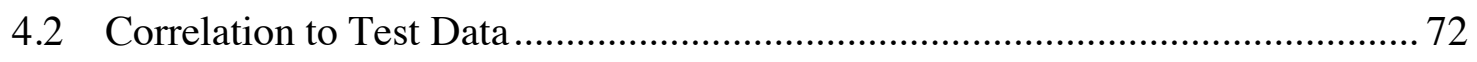

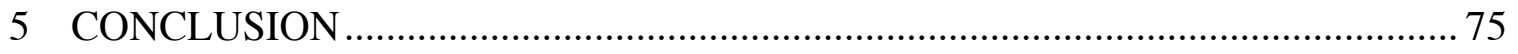

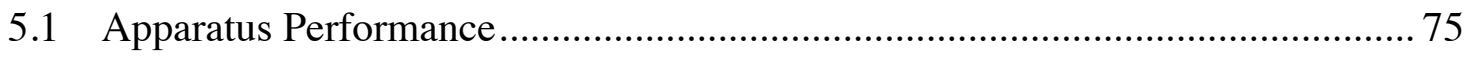

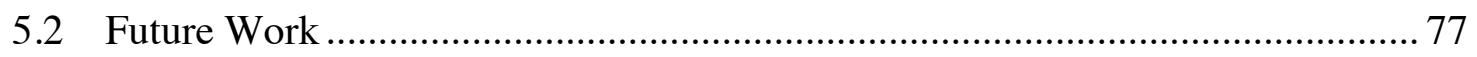

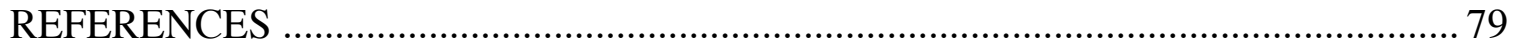

\section{APPENDICES}

A Operating Instructions .................................................................................. 82

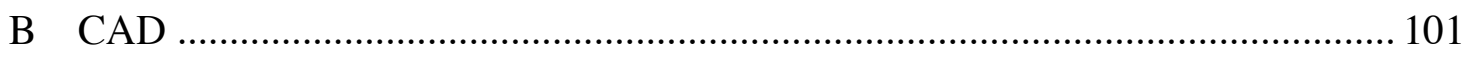




\section{LIST OF TABLES}

Table

Page

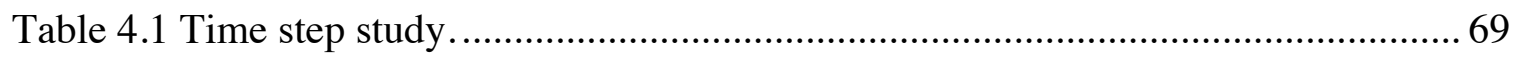

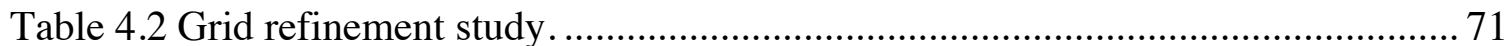




\section{LIST OF FIGURES}

Figure

Figure 1.1 Acceleration time history for shock event ............................................ 2

Figure 1.2 Example Shock Response Spectrum. ..................................................... 4

Figure 1.3 Acceleration time history with zero shift. [5] ....................................... 6

Figure 2.1 SDOF to Shock Response Spectrum..................................................... 17

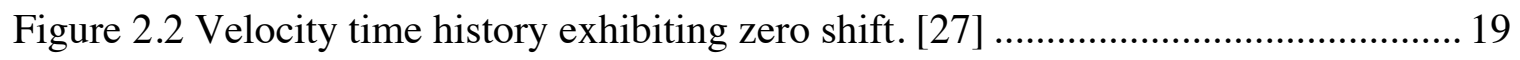

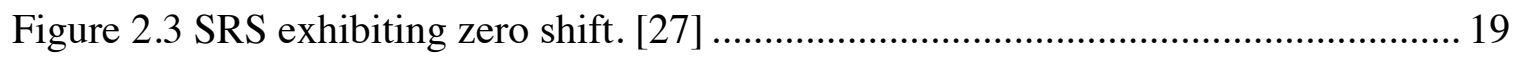

Figure 2.4 Comparison between Irvine and author SRS codes. ................................... 20

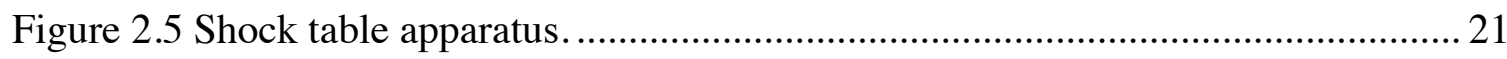

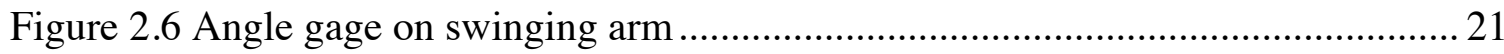

Figure 2.7 Repeatability plate mounted to pendulum............................................. 23

Figure 2.8 Floor anchor solution to immobilize shock table apparatus......................... 23

Figure 2.9 New hammer with weights attached.................................................... 25

Figure 3.1 Out of plane and in plane impact directions......................................... 27

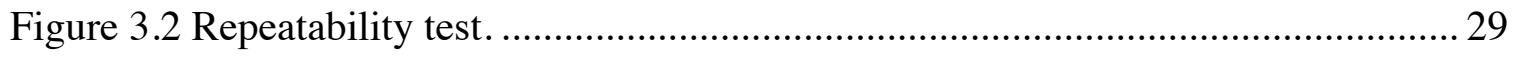

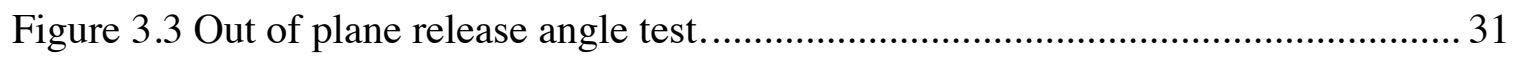

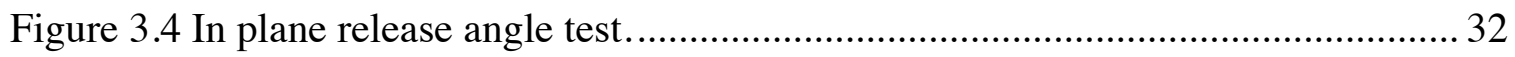

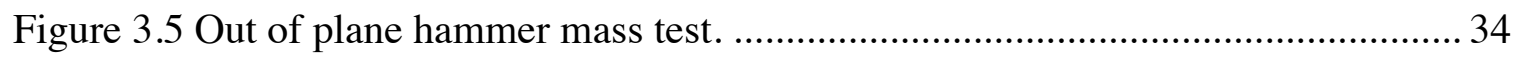

Figure 3.6 Out of plane hammer mass test with mounted test piece. ........................... 35

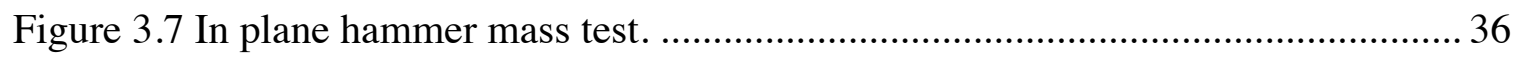

Figure 3.8 Out of plane hammer head diameter test................................................ 38

Figure 3.9 Out of plane hammer head material test................................................ 39 


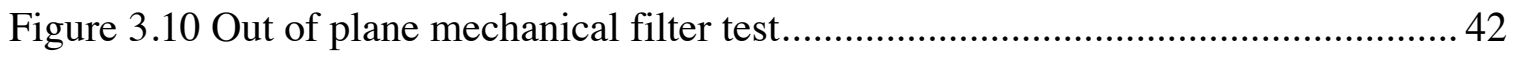

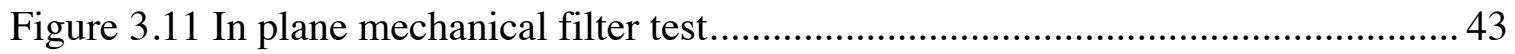

Figure 3.12 Accelerometer locations for out of plane sensor location test..................... 45

Figure 3.13 Out of plane sensor location test. ....................................................... 46

Figure 3.14 Accelerometer locations for in plane sensor location test. ........................ 47

Figure 3.15 In plane sensor location test. ........................................................ 47

Figure 3.16 Impact locations for the out of plane impact location test......................... 49

Figure 3.17 Out of plane impact location test....................................................... 50

Figure 3.18 Impact locations for in plane impact location test................................... 51

Figure 3.19 In plane impact location test....................................................... 51

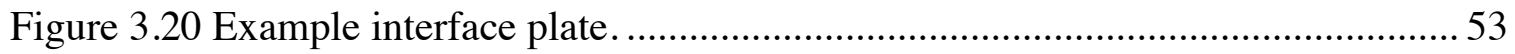

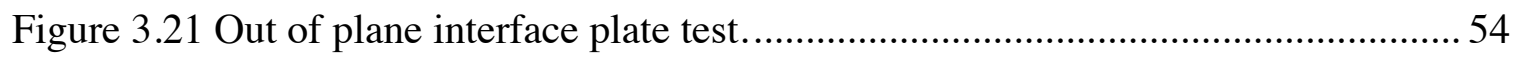

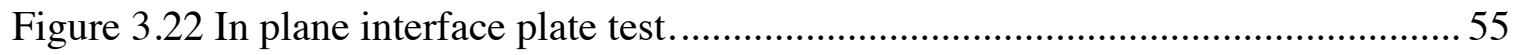

Figure 3.23 Out of plane test piece mass test........................................................... 57

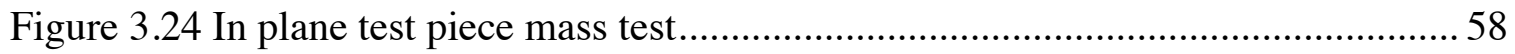

Figure 3.25 Test-POD mounted to shock table ....................................................... 59

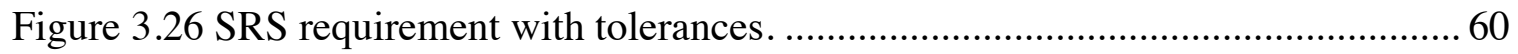

Figure 3.27 First out of plane attempt with the Test-POD...................................... 61

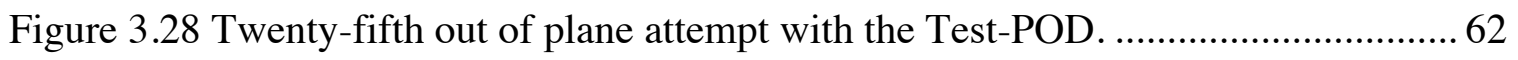

Figure 3.29 Fifty-seventh out of plane attempt with the Test-POD. ............................. 63

Figure 3.30 Sixty-fifth out of plane attempt with the Test-POD................................ 63

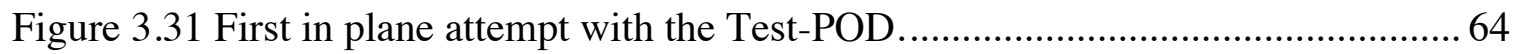

Figure 3.32 Fifteenth in plane attempt with the Test-POD....................................... 65 


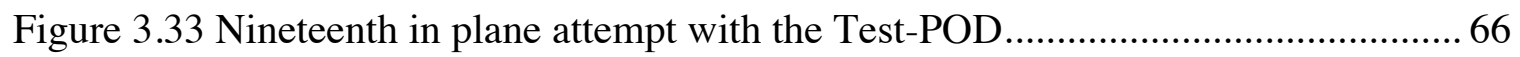

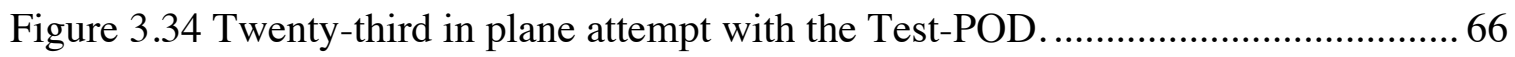

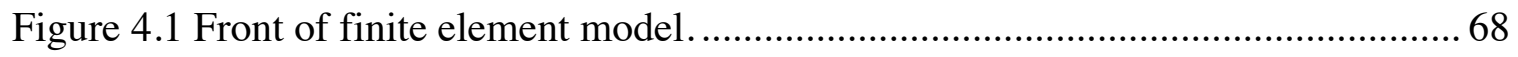

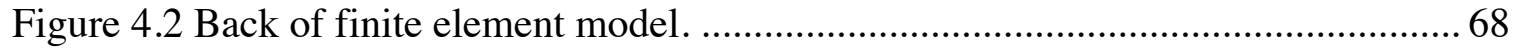

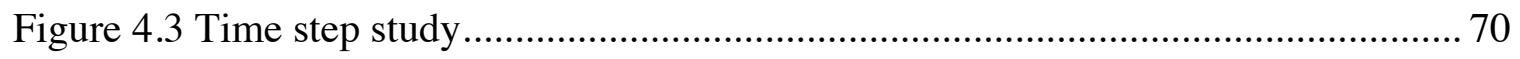

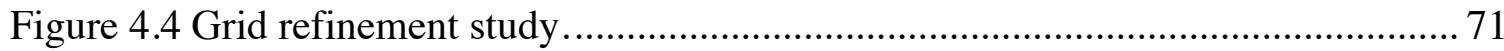

Figure 4.5 FEA of unloaded test that met SRS requirement...................................... 72

Figure 4.6 FEA of Test-POD test that met SRS requirement................................... 73

Figure 4.7 FEA of L-shaped interface compared to SRS requirement. .......................... 74 


\section{INTRODUCTION}

\subsection{Statement of Problem}

Spacecraft are subject to a variety of harsh environments throughout their lifetime that require testing be done to ensure survival. Each type of environment presents unique challenges that must be overcome to remain operational for the duration of a mission. These challenges extend to the testing, as each environment necessitates very different procedures and equipment. One such environment that must be simulated prior to the beginning of a mission is shock. Spacecraft experience shock events for a variety of reasons including fairing separation, spacecraft separation from launch vehicle, deployment of solar panels and other appendages, and the operation of pyrotechnic valves in the propulsion system. These shock events are generally initiated by pyrotechnic devices and are prevalent on virtually all space missions. The Mercury program had 46 installed pyrotechnic devices, Saturn had approximately 150, Apollo had in excess of 300 [1], and the Mars rovers Spirit and Opportunity each fired 126 pyro devices during atmosphere entry, descent, and landing alone. [2] Pyrotechnic devices are essentially controlled explosives and introduce a large amount of energy very quickly. The energy contains mostly high frequency content and thus does not usually harm structural members. However, the high frequency energy can pose a threat to crystals, brittle epoxies, glass diodes, and wire leads in the form of cracks, loss of seals, bond fractures, and electrical shorts. [3] Thus, shock testing must be done to verify that none of these failures will occur after a pyrotechnic device is fired.

The spacecraft programs at California Polytechnic State University-San Luis Obispo (Cal Poly-SLO) can currently perform all testing to qualify a spacecraft for 
launch other than shock testing. The addition of an adequate shock test facility would allow all of the testing to be done on campus as well as allow shock testing to be introduced to students in the Space Environments Lab curriculum. A shock test apparatus had been built on campus by a previous group of students and modified by a second group of students. However, neither group made enough progress to allow the shock test apparatus to be used for real mission testing, as will be discussed in the Literature Review section of this chapter. This paper will detail the changes made to the shock test apparatus that allow it to meet industry prescribed shock levels and tolerances, thus allowing it to perform actual shock testing to qualify spacecraft for launch.

\subsection{Shock Environment}

A shock is a sudden acceleration caused by an impact or explosion. Shock accelerations introduced by the firing of a pyrotechnic device (pyroshock) tend to oscillate about zero and follow an exponentially decaying envelope, as shown in Figure 1.1 .

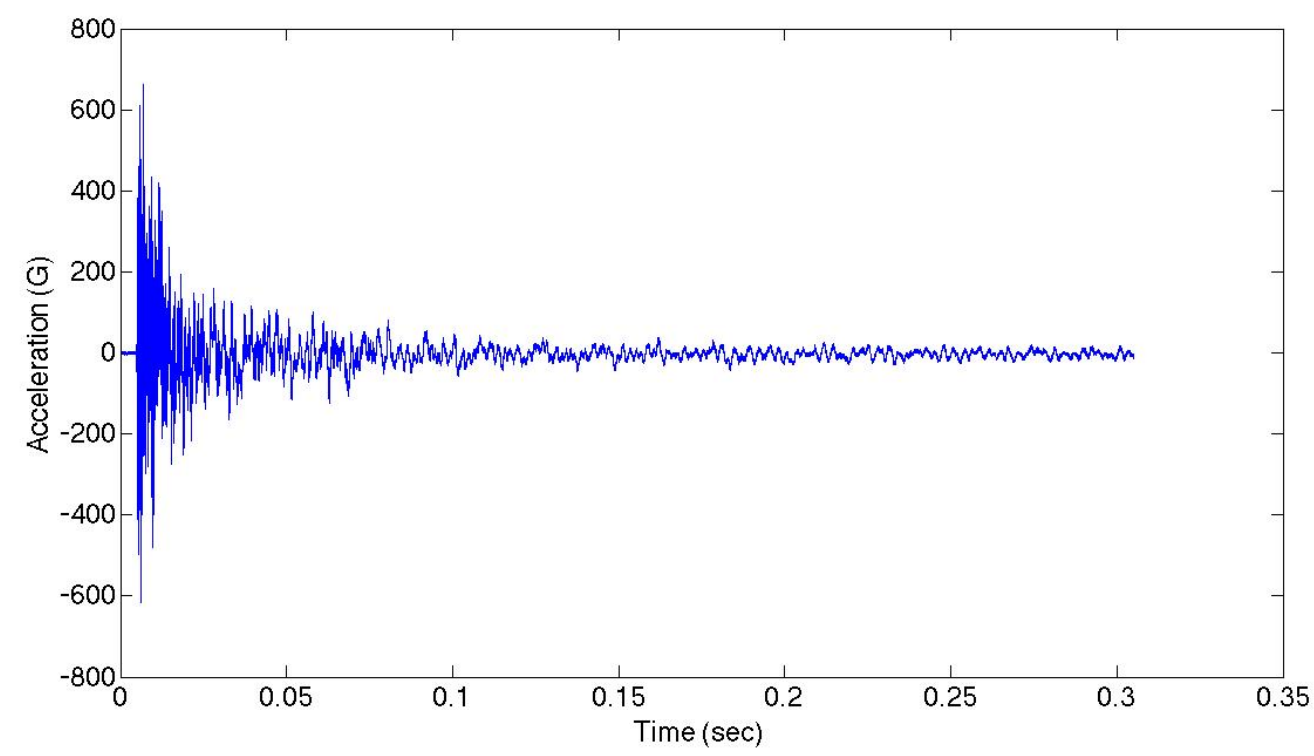

Figure 1.1 Acceleration time history for shock event. 
The acceleration time history for a pyroshock event is a very complicated waveform. If for example a launch vehicle provider gave a time history like the one in Figure 1.1 as the specification for the fairing separation, it would be virtually impossible to replicate that time history without the exact same equipment. This would be prohibitively difficult and expensive, as it would require all spacecraft to undergo a real fairing separation to ensure that it was subjected to the exact same shock environment. Every single pyrotechnic device on the spacecraft would have to be fired to replicate that particular shock environment.

Industry has developed an alternative to this thorough to a fault form of testing. A pyroshock event's acceleration time history is converted to a Shock Response Spectrum (SRS) that is then given as the specification. A Shock Response Spectrum is believed to give a good indication of the damage potential of the shock. [4] Very different shock waveforms can give the same SRS, making it a better specification to use in shock testing because different test setups with different equipment that produce a different waveform from the actual shock event can still be used for testing as long as the SRS is similar. The SRS corresponding to the time history shown in Figure 1.1 is given in Figure 1.2.

Since acceleration is the variable of interest in shock testing, accelerometers are the predominant sensor used in shock testing. Accelerometer design and purpose varies widely and it is important to select an accelerometer capable of withstanding and accurately capturing severe shocks. Shocks are sometimes classified into one of three categories: near-field, mid-field, and far-field. Near-field shocks have amplitudes in excess of 10,000 G (multiples of Earth's gravity) and significant frequency content well above $10 \mathrm{kHz}$. Explosives testing (simulating pyroshock events with an actual explosion) 
easily surpasses these levels, but close range metal-to-metal impact testing can also have significant high frequency content. [5] Most shock accelerometers can withstand midfield and far-field shocks, whose categorization can be easily found in the literature. [6,7]

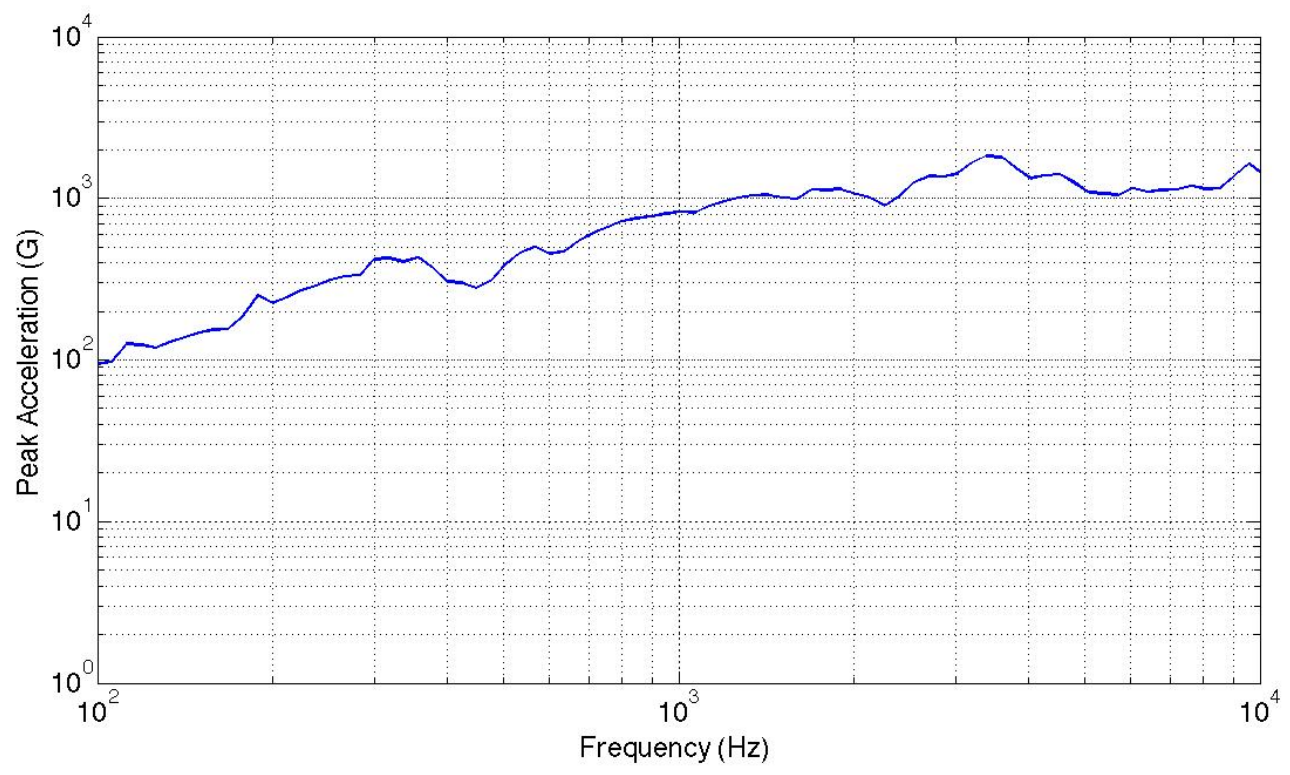

Figure 1.2 Example Shock Response Spectrum.

\subsection{Shock Testing}

Not only do spacecraft designers have a difficult task in designing a spacecraft to withstand the shock environment, they also must ensure that the testing is a reasonably accurate representation of that environment. Shock events often have large peak accelerations, necessitating accelerometers capable of surviving such harsh conditions. This turns out to not be too much of an issue as many accelerometer manufacturers offer models capable of surviving 50,000 $\mathrm{G}$ or more. The real issue lies in the high frequency content of the energy introduced by the shock event. Piezoelectric accelerometers use crystals as their sensing element and if the crystal's resonant frequency is excited by the shock it could damage the crystal itself. The most common problem in shock testing is a zero shift of the raw acceleration data, an example of which can be seen in Figure 1.3. As 
the name implies, a zero shift occurs when the accelerometer momentarily no longer reads zero after a shock event has dissipated. This indicates that a problem occurred while collecting data and usually renders the data unusable. Six causes of zero shift have been identified: overstressing of sensing elements, physical movement of sensor parts, cable noise, base strain induced errors, inadequate low-frequency response, and overloading of signal conditioner. [8]

Since zero shift corrupts accelerometer data, it should be avoided. One way to avoid it would be to prevent the accelerometer from being exposed to shocks that excite the sensing element's natural frequency. This may not be feasible as the shock required to meet the SRS specification might necessitate enough high frequency content to excite the sensing element. The most elegant solution to this problem involves choosing accelerometers with mechanical low-pass filters built in. These mechanical filters will attenuate the acceleration input to the sensing element above a certain frequency, thus preventing the resonance from being excited. This should help mitigate the first, second, and fifth causes of zero shift identified above. Using low noise cable between the accelerometers and the signal conditioner can reduce cable noise. Base strain induced errors can be avoided by following the accelerometer manufacturer's guidelines for both flatness of the mounting surface as well as torque specifications for those accelerometers with a built in mounting stud. The odds of overloading the signal conditioner can be reduced by choosing an accelerometer whose output voltage matches the signal conditioner's input voltage, making sure that the accelerometer will not be exposed to peak shock values greater than it was designed for, and choosing a signal conditioner with a high frequency response large enough to handle the shock event. 


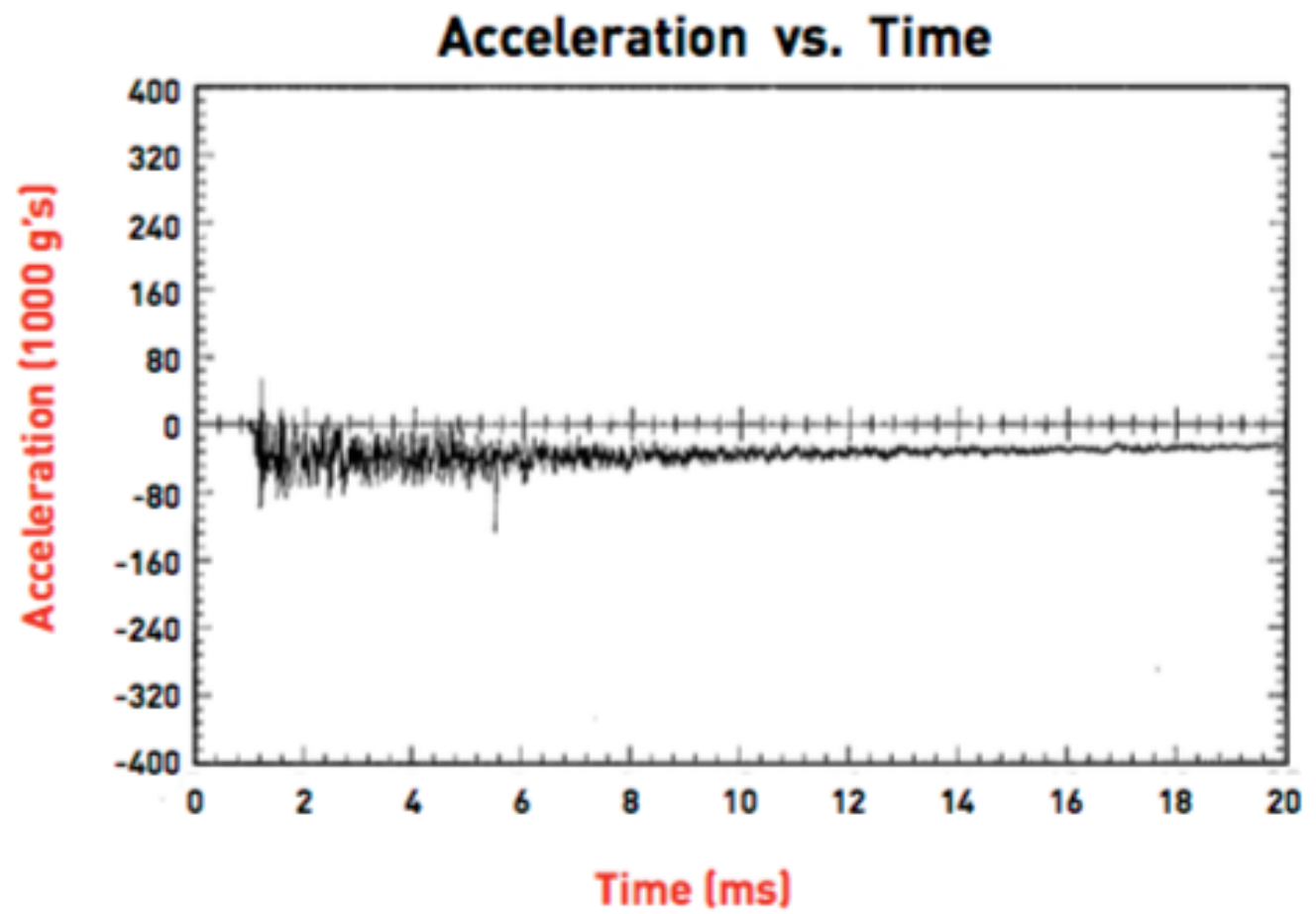

Figure 1.3 Acceleration time history with zero shift. [5]

\section{$1.4 \quad$ Literature Review}

Many shock test facilities exist in industry that employ a variety of techniques for simulating a pyroshock event. The Dornier Shock Table [9] utilized a horizontal aluminum plate that sat on a frame. The plate was excited by a mass that was dropped through a tube. The mass and impacting object material could be changed to modify the resulting shock response spectrum, and the test piece was mounted to the aluminum plate. Sandia National Laboratories used a pneumatically actuated piston to accelerate a sled along a set of rails. [10] The test piece was mounted to an initially stationary sled that was impacted by the sled that the piston accelerated. The shock response spectrum could be changed by modifying the portions of the sleds that touched each other during the collision and by changing the force exerted on the moving sled by the piston. The MGA Technical Services Laboratory used an air gun as its impact method. [11] The air gun 
impacted a resonant beam in the transverse direction. The test piece was mounted to the resonant beam, and various SRS curves could be achieved by changing the speed of the air gun projectile and by changing the length and cross section of the resonant beam. Mecano-ID excited a Hopkinson bar with a pendulum hammer. [12] A Hopkinson resonant bar is similar to a resonant beam, but it is impacted in the longitudinal direction instead of the transverse direction. Mecano adjusted the SRS by adding oscillators to the bar in order to excite additional response frequencies.

Those shock test facilities most similar to the shock test apparatus discussed in this paper utilize a pendulum hammer to excite the system. Martin Jonsson created a similar project in his paper "Development of a Shock Test Facility for Qualification of Space Equipment" [13] that proved to be very helpful. His shock table's frame was made of the same extruded aluminum framing. A large aluminum plate was excited with a pendulum hammer, just as in this project. A major difference involves the aluminum plate's orientation: this paper's plate was oriented vertically while the plate in Jonsson's paper was parallel to the ground. This gives Jonsson's table more parameters that can be varied to tune the plate's response, but the ideas presented in his paper are applicable here. Jonsson's paper was based on the work of a group of engineers at the European Space Agency and referenced the results of their work heavily, thus providing two sources to validate the work presented here. Both papers discuss the trends seen when varying different parameters, which was a good reference when this project began varying parameters. Both papers were able to correlate a finite element model with their physical tables, providing the motivation for the finite element analysis attempted for this paper. 
As mentioned previously, this paper builds on the work done by two previous groups of students. Joseph Binder, Matt McCarty, and Chris Rasmussen describe the assembly of Cal Poly-SLO's shock table in their paper "Development of a Pyrotechnic Shock Simulation Apparatus for Spacecraft Applications.” [14] They considered three designs: a tunable resonant beam clamped at both ends and impacted by an air gun, a horizontal plate impacted by a movable pneumatic piston, and a vertical plate with many possible impactors (a pendulum hammer was ultimately chosen). They also chose the accelerometers, signal conditioner, and data acquisition device. While the accelerometers are believed to be barely adequate and the signal conditioner is believed to be qualified, the data acquisition device (DAQ) was woefully inadequate. Sample rates will be discussed in the next chapter, but the DAQ chosen by this group only sampled at $16 \%$ of the minimum suggested sample rate. Only one test was done with no indication of the parameters used to achieve the results.

Isaac Nitschke and Charles Williams continued the previous group's work, as described in their similarly named paper "Development of a Pyrotechnic Shock Simulation Apparatus for Spacecraft Applications." [15] They began by noting that the previous group's Matlab code to generate an SRS curve from raw acceleration data had not been validated. They discovered that the code was incorrect and made the necessary changes. The aluminum plate was originally suspended from the frame by steel cables, but that was not rigid enough to allow the hammer to rebound after its initial impact with the plate. A few iterations were tested before settling on a rigid attachment between the aluminum plate and the frame. No changes were made to the data acquisition system, thus continuing the inadequate sample rate. 


\subsection{Paper Outline}

The next chapter of this paper will introduce the industry's data acquisition standards that drove the DAQ selection and give more information about the data acquisition system. It will also briefly discuss the algorithm used to generate the SRS curves and will conclude with the changes to the shock table itself. Chapter 3 is arguably the most important section, as it will detail the results of the test campaign; that is, it will discuss how the SRS changes as different parameters are varied. It will also include the attempts to tune the SRS to an actual SRS requirement with a real test component mounted to the plate. Chapter 4 will address the development of the finite element model as well as its agreement with the experimental results. Finally, Chapter 5 will draw conclusions regarding the project's success and provide suggestions for future work. 


\section{STANDARDS AND APPARATUS IMPROVEMENT}

\subsection{Data Acquisition Standards and Design}

As the previous discussion on zero shift indicates, shock events are difficult to accurately capture and digitize. Care must be taken in selecting the sensor, the signal conditioner, the analog filter, and the DAQ, as a deficiency in any of those pieces could render the whole data acquisition chain ineffective. Even if the entire data acquisition system is adequate, care must still be employed to ensure that the sensor is not placed in an environment it was not designed for as it could damage the sensor and would likely result in bad data. After years of shock testing, industry has developed several standards to decrease the chances of collecting and using bad shock data. These standards are still evolving as sensor technology advances and causes of bad shock data are better understood.

\subsubsection{Accelerometer Specifications}

Since very intense metal-to-metal impact can fall into the near-field category of shocks, that shock environment should be in mind when selecting an accelerometer. Explosives testing can result in accelerations in excess of $100,000 \mathrm{G}$ in close proximity to the source. Metal-to-metal impact testing does not create shock levels that high, but placing the accelerometer very close to the impact location can subject the accelerometer to some fairly high-G transient shock behavior so it is a good rule of thumb to keep that in mind when selecting the accelerometer's maximum $\mathrm{G}$ range. Even for metal-to-metal impact testing, 10,000 $\mathrm{G}$ should be the minimum acceptable $\mathrm{G}$ range.

Frequency response is another important accelerometer characteristic. It is similar to sensitivity, which is the ratio of the sensor's electrical output to mechanical input. [16] 
Frequency response essentially describes how linearly the sensitivity changes as the shock frequency changes and is specified with a tolerance on that linearity. The SRS is typically calculated to $10 \mathrm{kHz}$ so this is often cited as the necessary upper limit with a tolerance of either 5 or $10 \%$. [17] Even though the SRS typically starts at 100 or even 10 $\mathrm{Hz}$, it is useful to have an accelerometer with a linear frequency response as close to DC $(0 \mathrm{~Hz})$ as possible. Some accelerometer manufacturers cite frequency responses with upper limits in excess of $50 \mathrm{kHz}$ and near-field shock events can have frequency content above $100 \mathrm{kHz}$, but it is argued in the literature that the maximum frequency where measurements are still meaningful is $20 \mathrm{kHz}$. [18] Therefore, it is recommended that a frequency response from DC to $20 \mathrm{kHz}$ with a tolerance of $5 \%$ be sought.

Exciting an accelerometer's resonance not only increases the sensitivity by several times and thus distorts the data, but it can also pose a damage threat to the sensing elements. As a result, accelerometer manufacturers have tried to design sensors with resonant frequencies as high as possible in an effort to simply avoid exciting these frequencies. While resonant frequencies approaching $100 \mathrm{kHz}$ have been achieved, this does not guarantee that resonances will be avoided. Accelerometers with built in electrical filters can help maintain linearity into higher frequencies, but may not be adequate to attenuate the sensing element's resonant frequency. Accelerometers can now be found with built in mechanical filters as well, which can help attenuate or prevent an excited resonance as well as protect the sensing elements from the damaging high frequency content of pyroshocks. A high resonant frequency in addition to built in electrical and mechanical filters are desirable traits for a shock accelerometer. 


\subsubsection{Signal Conditioner Specifications}

A signal conditioner's required characteristics vary slightly based on the type of accelerometer used. Accelerometer signals typically require amplification (applying a gain to the signal) prior to digitization. Integrated electronic piezoelectric (IEPE)

accelerometers have this amplification circuitry built into them and do not require a signal conditioner capable of amplification. IEPE accelerometers are used in this project.

Regardless of the type of accelerometer used, the signal conditioner must be capable of excitation. That is, it must be capable of providing a constant current power source to the accelerometers. All accelerometers have a minimum required current, typically around $2 \mathrm{~mA}$. However, as the maximum frequency and amplitude of the acceleration to be measured increase (as in shock events), the necessary current increases. The maximum likely required current can be calculated and provided with additional knowledge of the accelerometer sensitivity, cable length, and cable capacitance. [19]

Two more signal conditioner characteristics should be considered to prevent overloading and saturation. The signal conditioner should be capable of handling all of the shock event's frequency content, so it should have a bandwidth from DC to at least $100 \mathrm{kHz}$. High frequency, high amplitude signals strain a signal conditioner's analog output channels, whose performance is measured by slew rate. Industry recommends a slew rate in excess of $20 \mathrm{~V} / \mu \mathrm{s}$.

\subsubsection{Analog Anti-Aliasing Filter Specifications}

The presence of significant high frequency content presents significant problems in data acquisition, as discussed previously. Another issue posed is aliasing of data, which is often difficult to identify and can render data unusable. Aliasing occurs when an 
analog signal contains frequencies in excess of the Nyquist frequency. The Nyquist frequency is defined as half the sample rate. Signals that are created (a sinusoidal signal generator, for example) have the advantage of a known maximum frequency, and aliasing can be avoided by simply sampling at twice that known maximum frequency. Many signals are actually composed of varying levels of all frequencies [20], in which case increasing the sample rate cannot prevent aliasing, as there is no such thing as an infinite sample rate. In these cases an analog filter must be included prior to digitization. This filter ideally allows only the parts of the signal in a certain frequency band to pass through and attenuates the signal at all other frequencies. In a low pass filter, the transition from frequencies allowed to pass unchanged and frequencies that are attenuated is called the cutoff frequency. Then the sample rate is set such that the Nyquist frequency occurs far enough above the cutoff frequency that the unwanted frequencies have been sufficiently attenuated. Then they are dwarfed in amplitude by the signal frequencies that were allowed to pass through and no longer introduce significant error into the digitized signal.

Shock events not only contain all frequencies, but in the case of pyroshock events there are high amplitudes in the high frequencies. This leads to another filter characteristic that must be designed in addition to the cutoff frequency: the rolloff rate. Rolloff rate is given in units of $\mathrm{dB} /$ octave, with $\mathrm{dB}$ indicating amount of attenuation and an octave being a doubling of frequency. Note that in this paper a decibel (dB) refers to 20 times a base 10 logarithm as the sensor signal varies with voltage, not wattage. The rolloff rate typically recommended in industry has been $60 \mathrm{~dB} /$ octave. $[21,22,23]$ As an example, a filter with this rolloff rate and a cutoff frequency at $25 \mathrm{kHz}$ could be part of a 
system that measured at $100 \mathrm{kHz}$. That would place the $50 \mathrm{kHz}$ Nyquist frequency one octave above the filter's cutoff frequency. Therefore, there would be $60 \mathrm{~dB}$ of attenuation by the Nyquist frequency.

This suggestion is a bit arbitrary, as it does not include any recommendations as to how many octaves should be between the cutoff frequency and the Nyquist frequency. After the 2011 Shock and Vibration Symposium [24], this recommendation was restated as follows: a filter with an attenuation of $60 \mathrm{~dB}$ or greater for 12-bit systems and $80 \mathrm{~dB}$ or greater for higher resolution systems, at the Nyquist frequency.

\subsubsection{Sample Rate}

In ordinary data acquisition applications, the sample rate is set by the highest frequency of substantial content. The Nyquist frequency could be set at this value, which would set the minimum sample rate at twice this value (as the Nyquist frequency is half the sample rate). However, this is not the standard recommendation given by industry for shock testing data acquisition. Himelblau [23] argues that the SRS is essentially a time domain quantity. To minimize potential error in the SRS calculation, it is recommended to use a sampling rate of at least 10 times the highest frequency included in the SRS. This value is usually $10 \mathrm{kHz}$, yielding a minimum recommended sample rate of $100 \mathrm{kHz}$. It should be noted that a sample rate could also be derived with the new filter recommendation put forth at the 2011 Shock and Vibration Symposium [24]. The higher sample rate should be used between that sample rate and the older $100 \mathrm{kHz}$ recommendation. 


\subsubsection{Selected Data Acquisition Equipment}

The chosen sensor was a Dytran 3200B4 IEPE accelerometer. The signal conditioner was a PCB Model 482C05. A Microstar Laboratories MSXB048-03-25KE2C-B filtered analog input expansion board served as the filter. A Microstar Laboratories DAP5380a/526 data acquisition processor completed the system as the DAQ. It should be noted that the signal passes between the equipment in the order they are presented in this paragraph (originating at the accelerometer and ending at the DAQ).

The Dytran accelerometer is rated up to $10,000 \mathrm{G}$ and can survive in excess of $50,000 \mathrm{G}$, so it barely meets the range suggestion. Its frequency response with a $5 \%$ tolerance runs from 0.5 to $10,000 \mathrm{~Hz}$, and with a $10 \%$ tolerance runs from 0.35 to 20,000 Hz. It comes close to meeting the recommended frequency response. It has a resonant frequency in excess of $90 \mathrm{kHz}$. It contains a built in electrical filter, but no mechanical filter. At the beginning of this project the Dytran accelerometer was deemed barely adequate but an upgrade was desired. The PCB 350C02 or 350D03 were deemed to be better fits, but budget constraints prevented their acquisition.

The PCB signal conditioner is designed for IEPE applications, meaning that it can provide a current source to the accelerometers. Its output current range perfectly matches the accelerometers' input current range. The fact that the signal conditioner acquires the signal prior to the analog filter initially led to worries of overloading the signal conditioner. However, its bandwidth of greater than $1 \mathrm{MHz}$ is theorized by this author to be adequate for the metal-to-metal impact testing that was conducted. The manufacturer quoted the slew rate as being between 20 and $30 \mathrm{~V} / \mu \mathrm{s}$, which is sufficient. Overall, the author was satisfied with this piece of the data acquisition chain. 
The two Microstar Laboratories pieces of equipment combine to filter and digitize the data. The filter board contains a 4 pole Butterworth filter with a cutoff frequency at $25 \mathrm{kHz}$. Butterworth filters typically achieve a rolloff rate of $6 \mathrm{~dB} /$ octave/pole, giving this filter a rolloff rate of about $24 \mathrm{~dB} /$ octave. The DAP board can sample the system at up to $800 \mathrm{kHz}$ and samples at 14-bit resolution. This places it in the category of $80 \mathrm{~dB}$ of attenuation by the Nyquist frequency. With the filter's $24 \mathrm{~dB}$ /octave rolloff rate, 3.33 octaves are required to meet that recommendation. This places the Nyquist frequency at 3.33 octaves above the filter's $25 \mathrm{kHz}$ cutoff frequency, which is about $250 \mathrm{kHz}$. Therefore, the minimum sample rate should be $500 \mathrm{kHz}$, which the DAP board is capable of achieving.

\subsection{Analysis Standards and Design}

After collecting the acceleration time history, the next step is to generate the SRS. A Shock Response Spectrum is formed by applying an acceleration time history as a base excitation to a series of single degree of freedom (SDOF) mass-spring-damper systems. Each system is assumed to have the same damping, with $5 \%$ being a typical number. The series of SDOF systems will cover a specified range of natural frequencies. These natural frequencies usually follow a proportional bandwidth scheme such as 1/12 octave, meaning that each natural frequency is $2^{1 / 12}$ times the preceding natural frequency. The acceleration time history is applied to each SDOF system individually, and each system's peak absolute response is recorded. After recording enough peak responses to cover the frequency range of interest (typically from $100 \mathrm{~Hz}$ to $10 \mathrm{kHz}$ ) the peak responses are plotted versus their corresponding natural frequencies. This plot is an SRS and it usually has a log scale on both axes. Figure 2.1 gives an example of the process. 

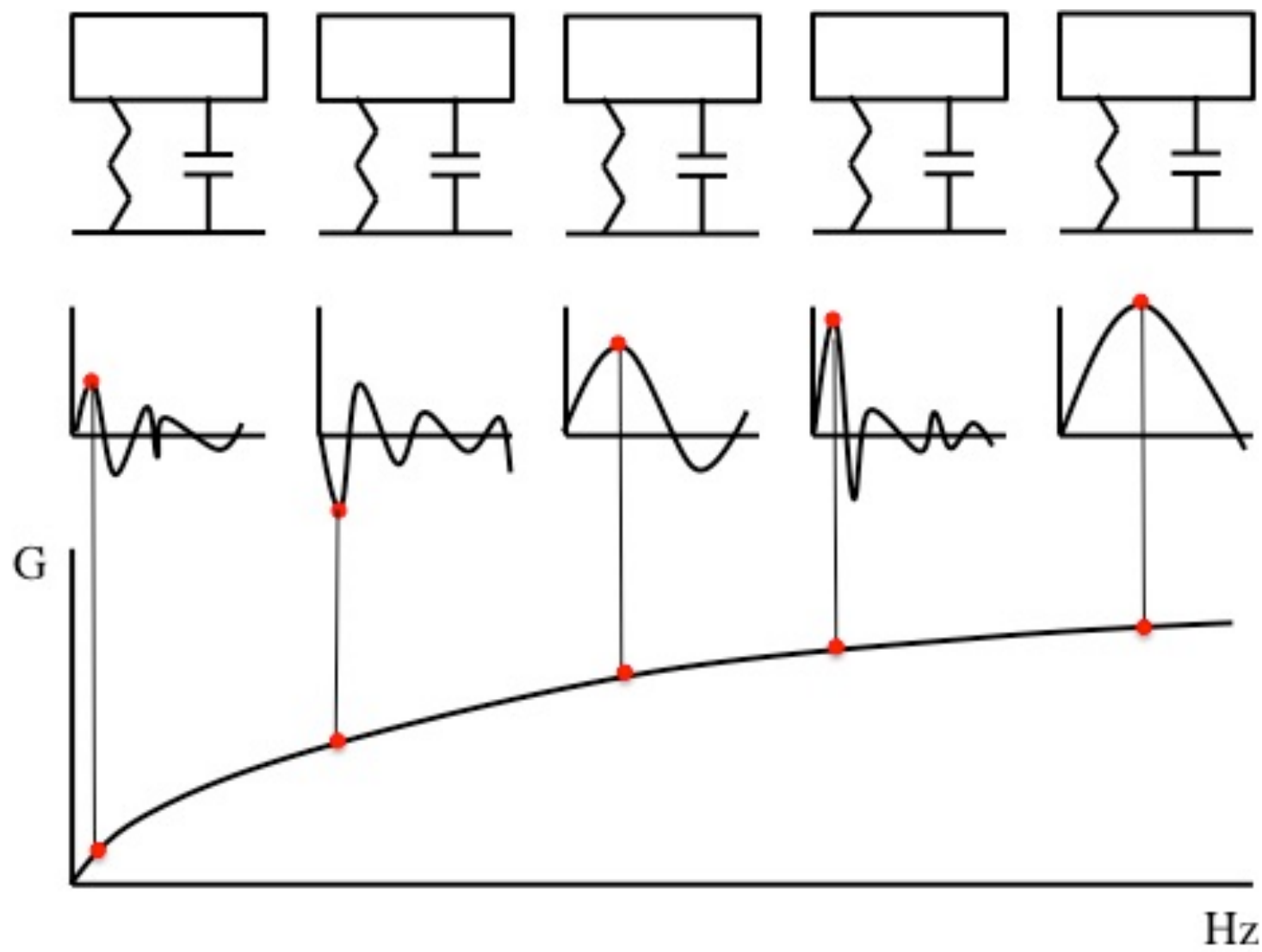

Figure 2.1 SDOF to Shock Response Spectrum.

\subsubsection{SRS Algorithm}

There are many ways to carry out the process depicted in Figure 2.1. Kelly and Richman discussed several methods [25] and Smallwood [26] discussed the most widely used algorithm today. The algorithm used by this author is derived by Irvine in Appendix A [27]. It was chosen because it is an efficient, straightforward algorithm that makes use of digital recursive filtering techniques (Smallwood described this category of algorithms [26]).

\subsubsection{Processing Data}

Himelblau [23] suggests that some type of correction be made for any zero offset that may exist in the data. Even a small zero offset can result in significant errors in the 
low frequency portion of the SRS. The signal conditioner used in this system has a stated DC offset of no more than $20 \mathrm{mV}$. This may seem trivial, but with an accelerometer sensitivity of $0.5 \mathrm{mV} / \mathrm{G}$, this translates to possibly a $40 \mathrm{G}$ offset. This would be more than enough to distort the lower frequencies in the SRS. This can be avoided by averaging the data points prior to the shock event and subtracting that value from the entire data set. An alternative to this is to average all of the data points and to subtract that value from the entire data set.

\subsubsection{SRS Acceptance}

The first stage of accepting an SRS should be examining the data to make sure it is good quality. This project used piezoelectric accelerometers, and as discussed in section 1.3 the main source of error in shock testing with these accelerometers is zero shift. The zero shift will not always be as obvious as in Figure 1.3. Since it represents such a danger to the data quality, instances of zero shift must be found. The data must pass three quality checks before being accepted as possibly reasonable. The first is a visual inspection of the raw acceleration data. The second is a visual inspection of the velocity time history, which can be acquired by integrating the acceleration time history. A zero shift here can be identified as a rapid departure from zero in either direction, as shown in Figure 2.2. The third quality check is a comparison of the positive and negative SRS curves. The positive SRS curve is created with the maximum positive SDOF responses while the negative SRS curve is created with the absolute value of the most negative SDOF responses. These two curves should agree well across the entire frequency range of interest. A zero shift can be identified by a large divergence between the two curves, especially in the lower frequencies. Figure 2.3 presents an example. 


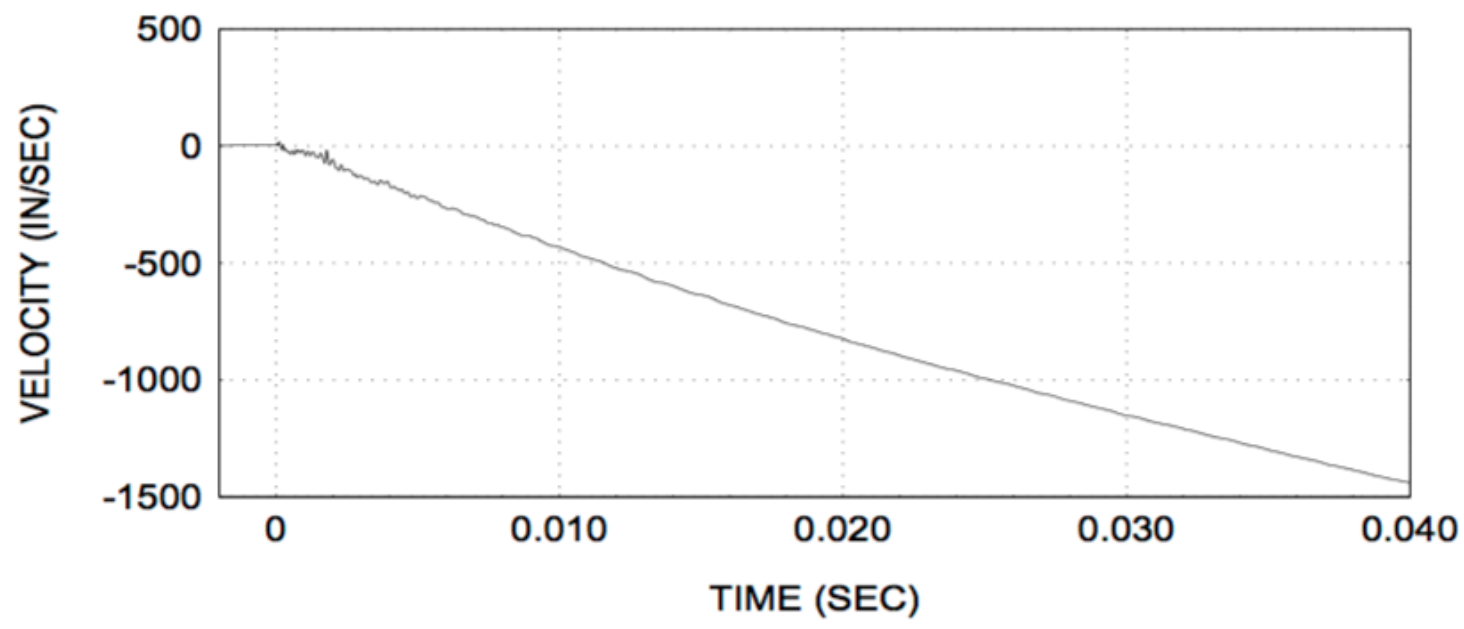

Figure 2.2 Velocity time history exhibiting zero shift. [27]

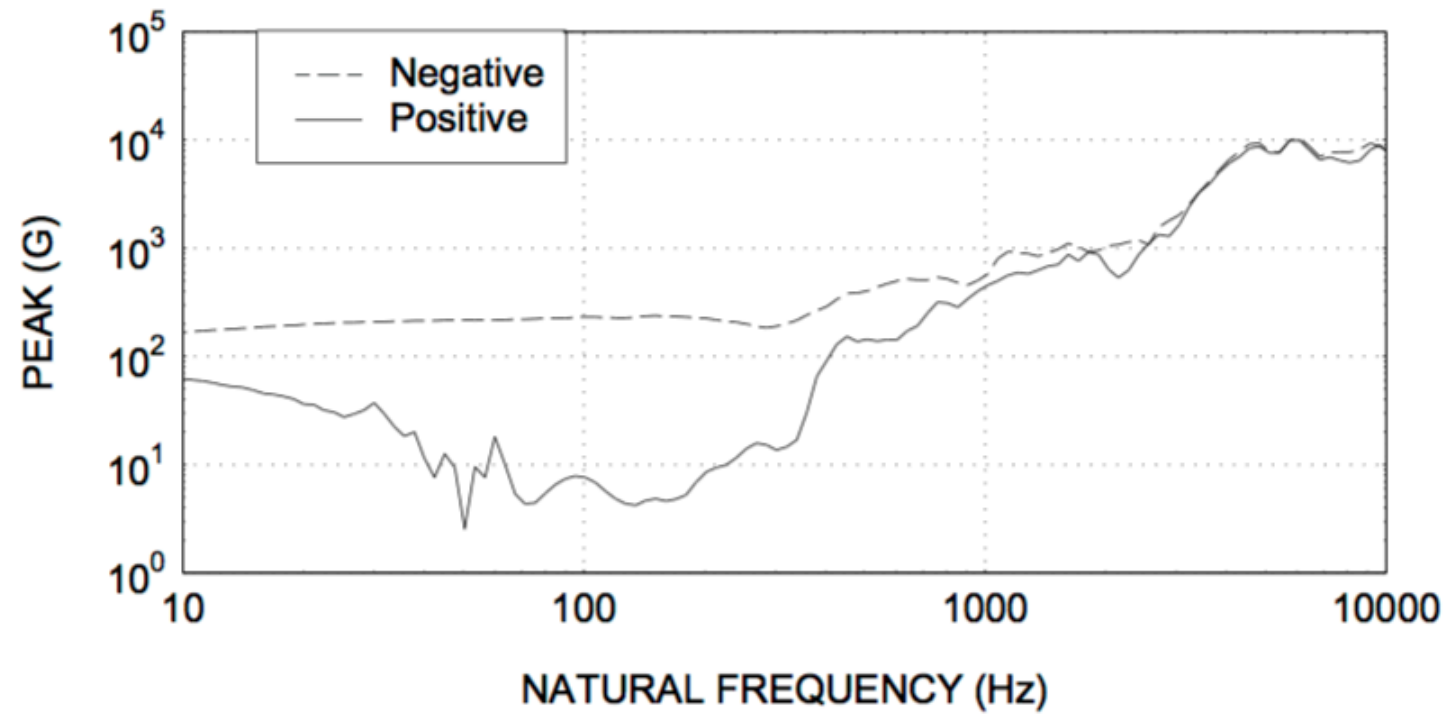

Figure 2.3 SRS exhibiting zero shift. [27]

Once it has been determined that the SRS was generated with quality data, it can be inspected relative to the launch vehicle provider required SRS. This will typically be stated with either a reference standard or a set of tolerances. The NASA GEVS 7000A tolerances are $\pm 6 \mathrm{~dB}$ for SRS frequencies less than or equal to $3 \mathrm{kHz}$, and $+9 /-6 \mathrm{~dB}$ for frequencies greater than $3 \mathrm{kHz}$. [28] The MIL-STD-810F tolerances require $+6 /-3 \mathrm{~dB}$ over at least $90 \%$ of the frequency band and $+9 /-6 \mathrm{~dB}$ for the remaining $10 \%$ of the 
frequency band, with that band running from $100 \mathrm{~Hz}$ to $10 \mathrm{kHz}$. [29] These tolerance values are applied to the specified SRS, and the SRS resulting from the test data must fall inside the tolerance bands. Both the GEVS and the Military Standard have an additional requirement: at least $50 \%$ of the SRS data points must exceed the nominal (launch vehicle provider specified) test level. If the SRS data passes the quality checks and tolerance requirements, then the test was successful and the spacecraft or component should be qualified for launch.

\subsubsection{Algorithm Validation}

Even if the data acquisition system collects good data, poor results could still be obtained with a poor SRS algorithm. Tom Irvine derived the algorithm used in this paper [27], and he has worked in shock and vibration in the aerospace industry since at least 1990. He maintains a website that includes Matlab code for generating an SRS. A comparison of an SRS generated with this Matlab code and the author's Matlab code is shown in Figure 2.4. Note that this SRS is the requirement to fly on a Falcon 9.

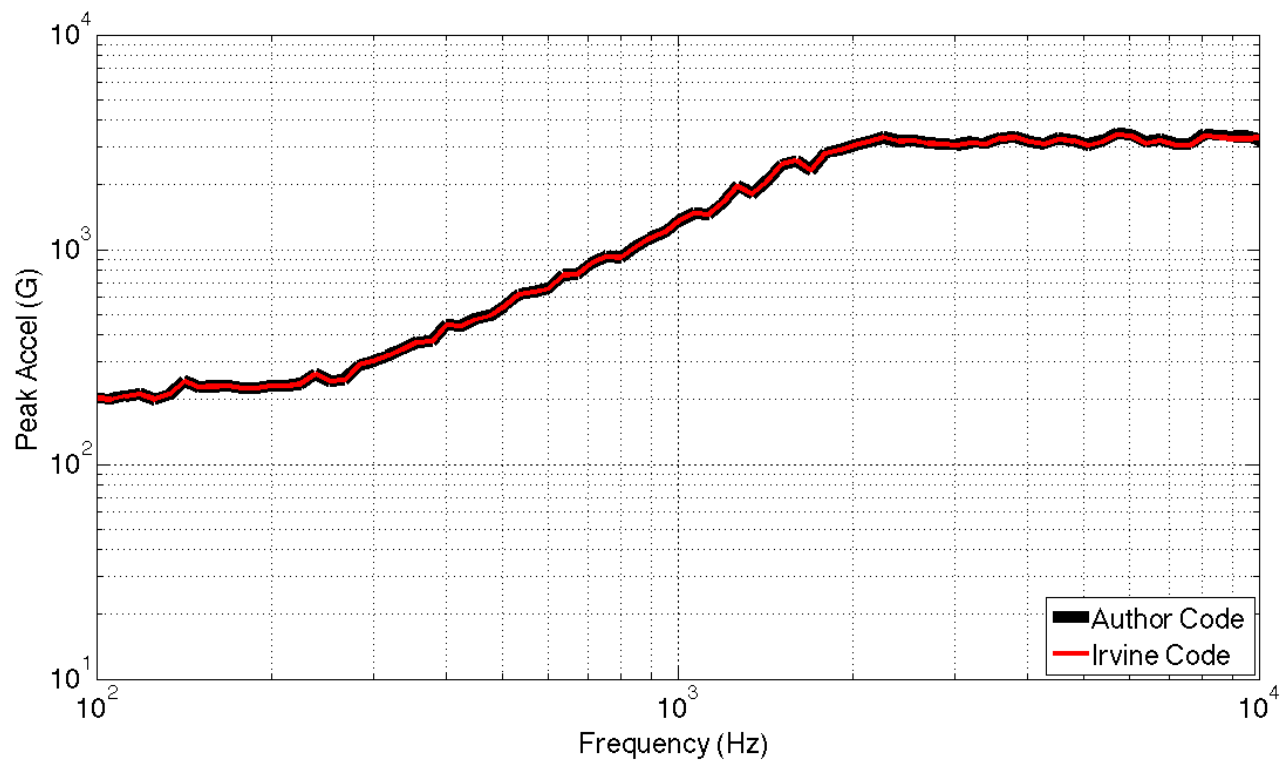

Figure 2.4 Comparison between Irvine and author SRS codes. 
The two sets of code produce a very similar SRS. The maximum percent difference between the two curves is only $4.1 \%$, and the average difference is only $0.4 \%$. The algorithms match better in the lower frequencies, with an average difference of $0.04 \%$ for frequencies below $2000 \mathrm{~Hz}$ and $1.0 \%$ above $2000 \mathrm{~Hz}$.

\subsection{Apparatus Design}

The author inherited the shock table apparatus in a functional state that required some modifications to provide accurate, repeatable results. At a very high level, the shock table design consisted of an aluminum plate suspended vertically from a frame that is impacted by a pendulum hammer. Figure 2.5 depicts this setup. The hammer frame at the front of the figure supports the hammer, which is pulled back and released to impart a shock to the aluminum plate. The apparatus is shown in the out of plane configuration, meaning that the impact occurs perpendicular to the plane defined by the aluminum plate.

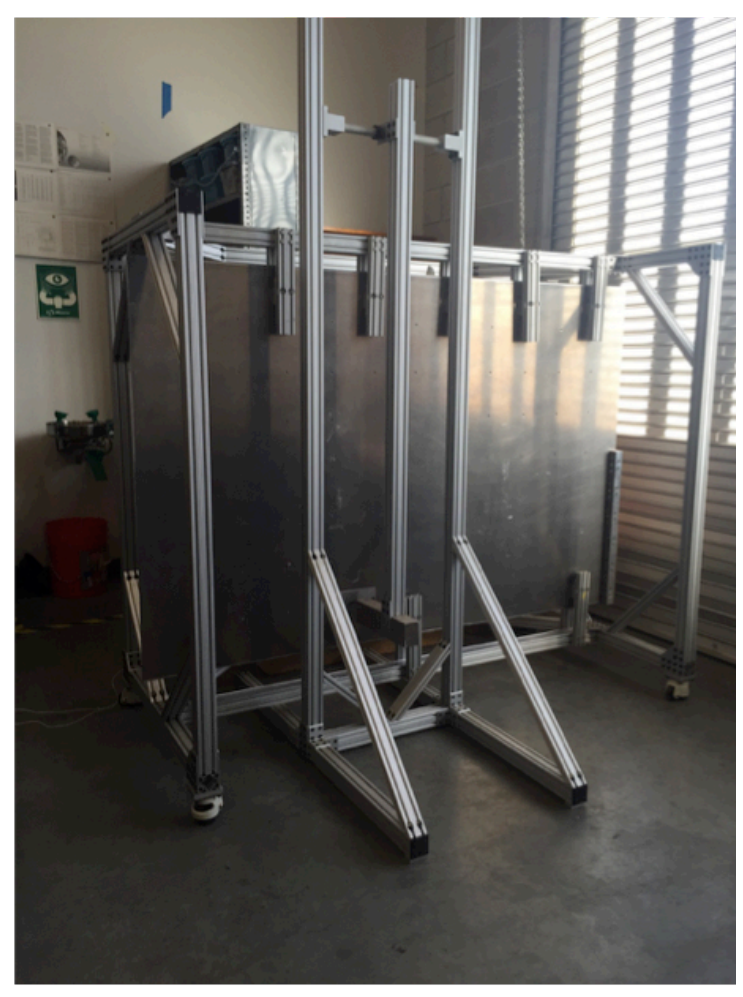

Figure 2.5 Shock table apparatus.

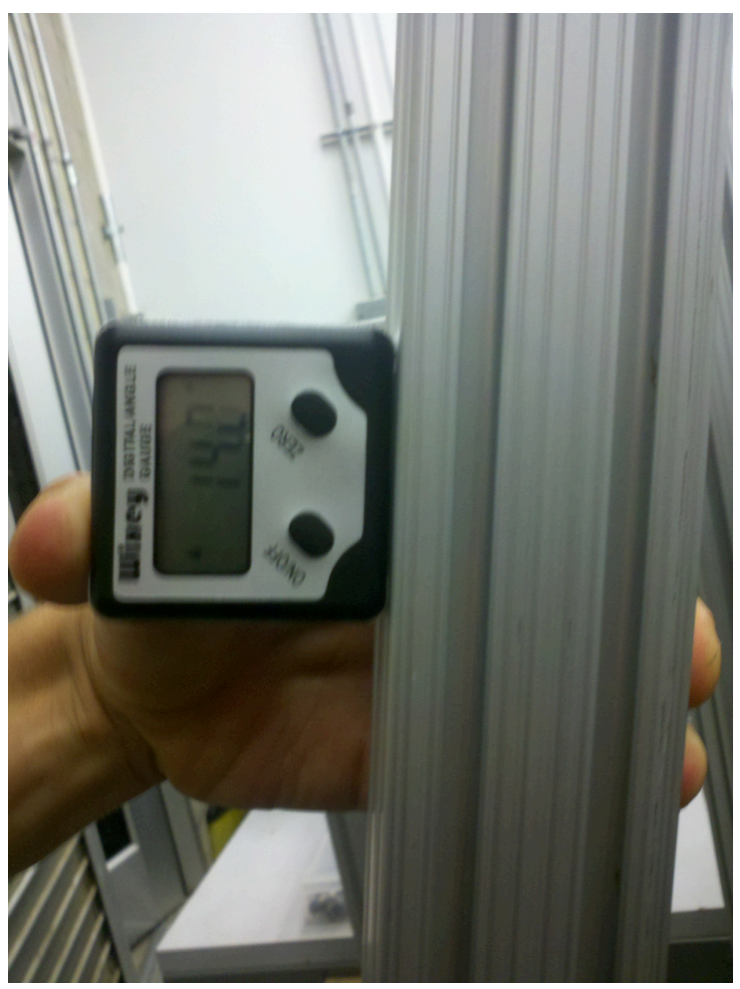

Figure 2.6 Angle gage on swinging arm 
Functionally, this design was fine. The plate is full of $1 / 4-20$ through holes in a 6inch square pattern for mounting test pieces. The pendulum can move relative to the table and the swinging arm can be slid up and down the hammer frame to allow for different impact locations. However, there were two major issues that prevented this design from performing tests at an industry-level standard.

The first problem involved the method of measuring the angle from which the swinging arm was released. As shown in Figure 2.6, the original design called for an angle gage to be held against the arm. Knowing the actual angle could be difficult as angle gages are fairly noisy and would need to be calibrated the exact same way every time. Exactly repeating a release angle from run to run would also be difficult, as it would depend on the operator's skill and again on any calibration changes between runs. The second problem was the fact that the frame suspending the aluminum plate was not fixed to anything. This allowed the entire assembly to move when impacted by the pendulum hammer, sometimes by large amounts when hit from a high angle. The SRS algorithm assumes a zero net velocity change and a zero relative displacement, so a moving assembly would not work. Even if the movement could be detected and removed from the data, it would make repeating a test run more difficult. Both problems were resolved as described in the next two sections.

\subsubsection{Repeatability Plate}

The angle gage method of determining release angle needed to be improved. The chosen solution involved the manufacturing of two identical plates to mount to the vertical pieces of the pendulum assembly. Each plate had 20 holes corresponding to

different swing arm angles. The plates were mounted just under the bar around which the 
swinging arm pivots, as shown in Figure 2.7. A metal bar placed in the corresponding holes in both plates served as a rigid stop against which the swinging arm could be pulled and released. This method yielded good repeatability results, as discussed in Chapter 3 .

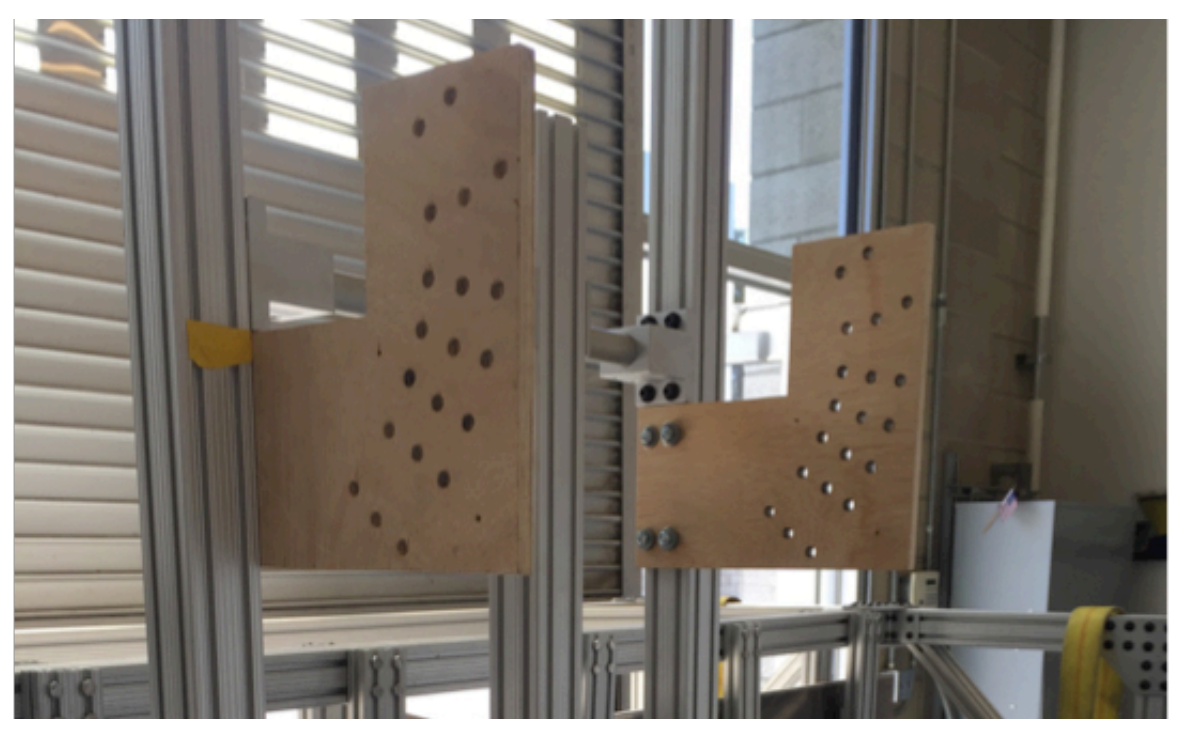

Figure 2.7 Repeatability plate mounted to pendulum.

\subsubsection{Floor Anchors}

Floor anchors were absolutely necessary to turn the existing shock test apparatus into a facility capable of performing industry quality tests. The anchor solution is shown in Figure 2.8.

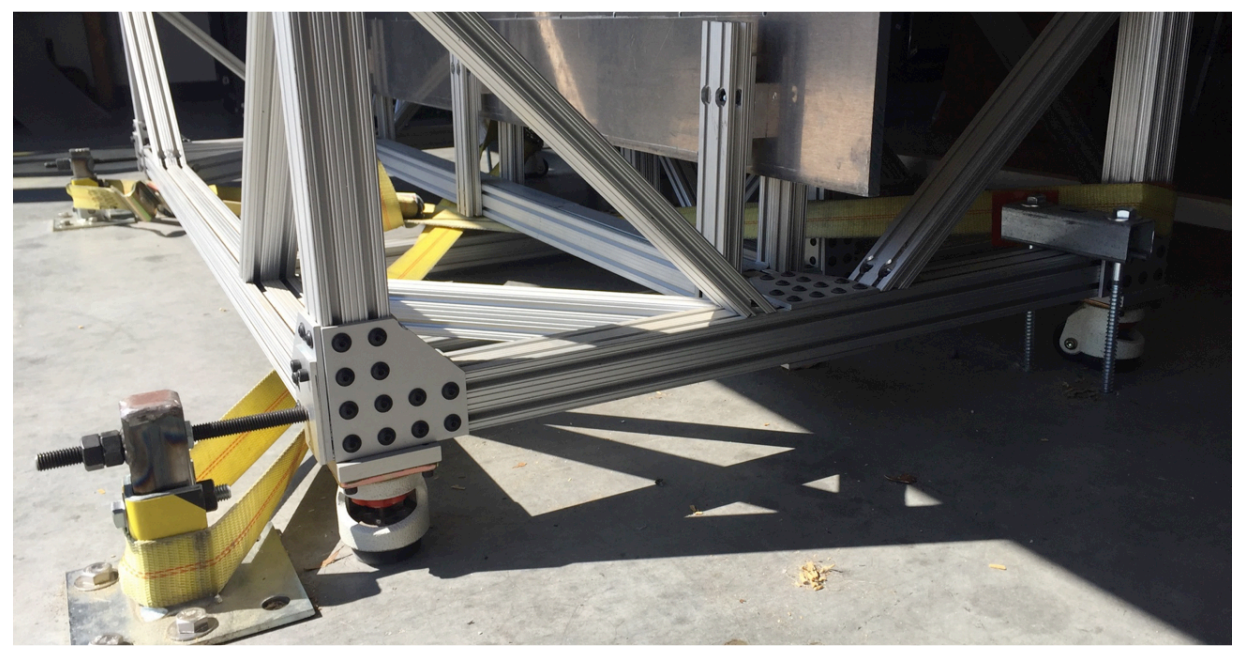

Figure 2.8 Floor anchor solution to immobilize shock table apparatus. 
In this figure the out of plane impact direction is from right to left. The two anchors on the left side are secured to the floor and prevent the apparatus from sliding to the left when impacted. The two anchors on the right side (only one is shown) prevent the apparatus from tipping when impacted. They combine to totally immobilize the shock table apparatus when impacted from right to left. The apparatus still moved a little bit when struck in the in plane direction (from into page to out of page) due to an oversight by the author. When commissioning the floor anchors, the author should have had floor anchors like those on the left side of the figure added to the front of the figure. This would have immobilized the apparatus for in plane strikes as well. The temporary solution was to add the heavy yellow straps visible in the figure. They do a marginally adequate job of immobilizing the apparatus in the in plane direction, but if that direction is to become a major part of testing going forward then more anchors like those on the left side of the figure should be added. As is the apparatus slides a small amount upon an in plane impact.

\subsubsection{New Hammer}

The original hammer consisted of a 2" x 3" x 12" block of steel weighing about 20 pounds. A one-inch hole in one of the small faces allowed a titanium hammer head to be inserted into the hammer and secured with a set screw. The titanium hammer head performed the actual impacting of the aluminum plate. The hammer served its purpose perfectly well, but several references $[13,30]$ suggest that varying the hammer weight allows influence over the SRS curve below about $1 \mathrm{kHz}$. As many ways of influencing the SRS as possible were desired, so a new hammer was manufactured. This hammer was smaller, 2" x 2" x 6", and only weighed about 7 pounds. It had the same method of 
securing the hammer head as the previous hammer. It also featured a large threaded through hole oriented parallel to the swinging arm. A threaded rod could be inserted into this hole and then weight could be added to the hammer. The new hammer is shown in Figure 2.9.

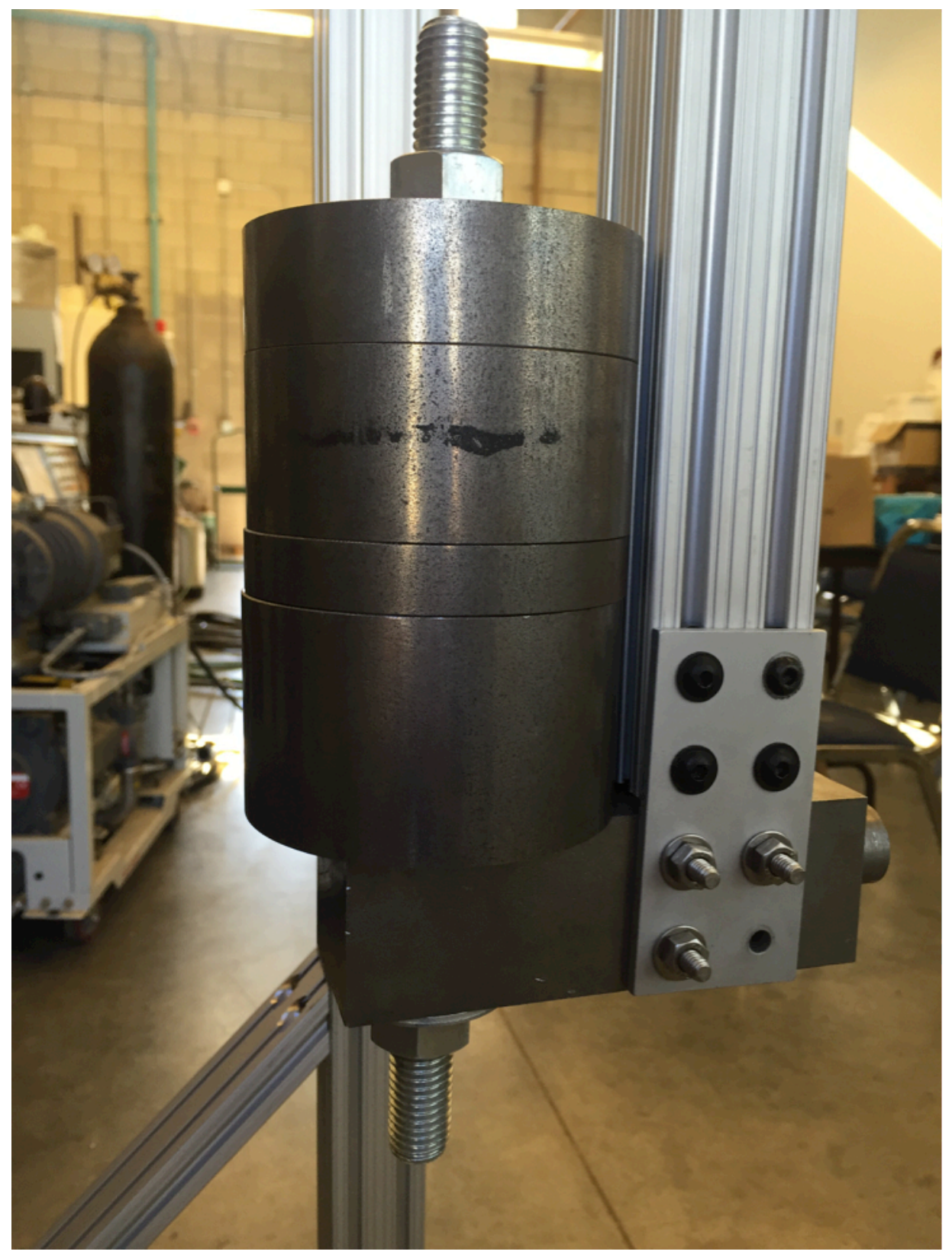

Figure 2.9 New hammer with weights attached. 


\section{TEST CAMPAIGN}

After improving the data acquisition system and shock table apparatus to a satisfactory level, a test campaign was carried out. This campaign involved collecting and processing data from over 200 tests with varying parameters. The chosen parameters were pendulum arm release angle, hammer mass, hammer head diameter, hammer head material, use of a mechanical filter at the point of impact, accelerometer location, impact location, presence of an interface for a test component, and the mass attached to that interface. It will be shown in this chapter that these parameters provide enough control of SRS shapes to meet a variety of SRS specifications.

The test campaign had several goals. The most obvious one was to understand how varying the above parameters affected the resultant SRS. Related to this was a need to characterize the shock table apparatus's basic behavior. A true pyroshock SRS should have approximately a 6-12 $\mathrm{dB} /$ octave positive slope in the lower frequencies and a flat plateau in the upper frequencies. The frequency where the positive slope transitions to the flat plateau is called the knee frequency. Every shock test apparatus will have slightly different slopes and knee frequencies, and these are difficult to shift more than one or two $\mathrm{dB}$ in any direction; they are essentially an inherent characteristic to the apparatus. Knowing these values will give an indication of a shock test facility's capabilities. If an SRS specification is very different from the apparatus's basic behavior, either the apparatus will likely have to be drastically changed (changing the resonant plate's length for example) or a different apparatus will need to be used.

In order to improve the user friendliness of this shock test facility, another goal was to generate a searchable database of all of the tests. Shock testing necessitates a large 
amount of trial and error to get an entire SRS inside the stated tolerances for a requirement SRS. This database would allow a user to input a desired SRS and the database would search all of the SRS curves generated from previous tests. After finding the SRS that most closely matched the desired SRS, the database would provide the user with the parameters used in the previous test. This would give the user a starting place for achieving the desired SRS and would ideally reduce the user's trial and error.

The test campaign's final main goal was to work out any issues with the data acquisition system and shock table apparatus and establish a "best practice" procedure for the facility's use. For example, the beginning of the test campaign resulted in many SRS curves that exhibited behavior like that shown in Figure 2.3: a large difference between the positive and negative SRS curves in the lower frequencies that indicated a zero shift. At this time the sample rate was only $200 \mathrm{kHz}$ instead of the minimum recommendation of $500 \mathrm{kHz}$. Once the sample rate was increased to an appropriate level, zero shift became a much less frequent issue.

The sections of Chapter 3 are broken into two parts: out of plane and in plane. This refers to the impact direction and the difference is shown in Figure 3.1.

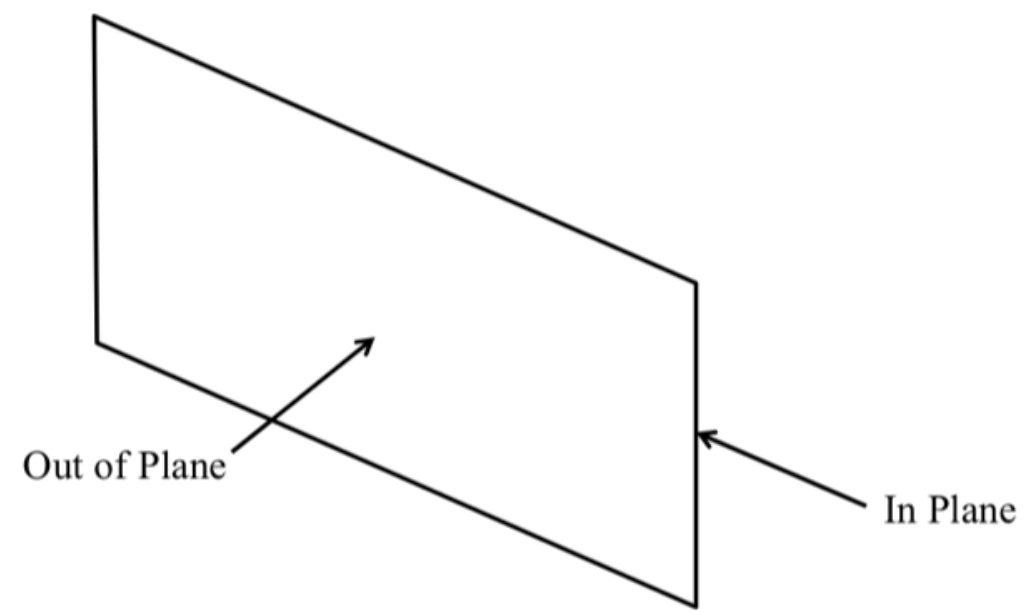

Figure 3.1 Out of plane and in plane impact directions. 


\subsection{Repeatability}

Having the ability to repeat results for a given set of parameters is important for two reasons. The first is that it allows a user to perform any equalization tests (tests to match the resulting SRS to the desired or specified SRS) on a dummy load so as to not damage the actual test piece from on an accidental over-test or from too many tests. After achieving the desired SRS with the dummy load, the shock test facility should be able to create virtually the exact same SRS with the test piece. The second reason is it allows for better characterization of how a parameter affects the SRS. Having good repeatability ensures that when changing a parameter, any resultant change in the SRS is due almost entirely to that parameter and not to natural variation in the shock table apparatus. This author defined repeatability as a maximum difference of less than $1 \mathrm{~dB}$ between any two tests with identical parameters. This decibel value was chosen because it was seen in the literature $[13,30]$.

With the anchoring of the shock table apparatus and the addition of a physical stop to improve the release angle consistency came an expectation of reliable repeatability. The two largest sources of error had been addressed and the author could now focus on details such as keeping all fasteners securely tightened and maintaining the pendulum hammer's position between runs. Paying attention to slight sources of variability such as these further increased the ability to repeat results.

To test the apparatus's repeatability, three identical tests with out of plane impacts were run in succession. The pendulum arm release angle was 12.9 degrees and the hammer did not have any extra mass attached. Figure 3.2 shows the repeatability test's results. 


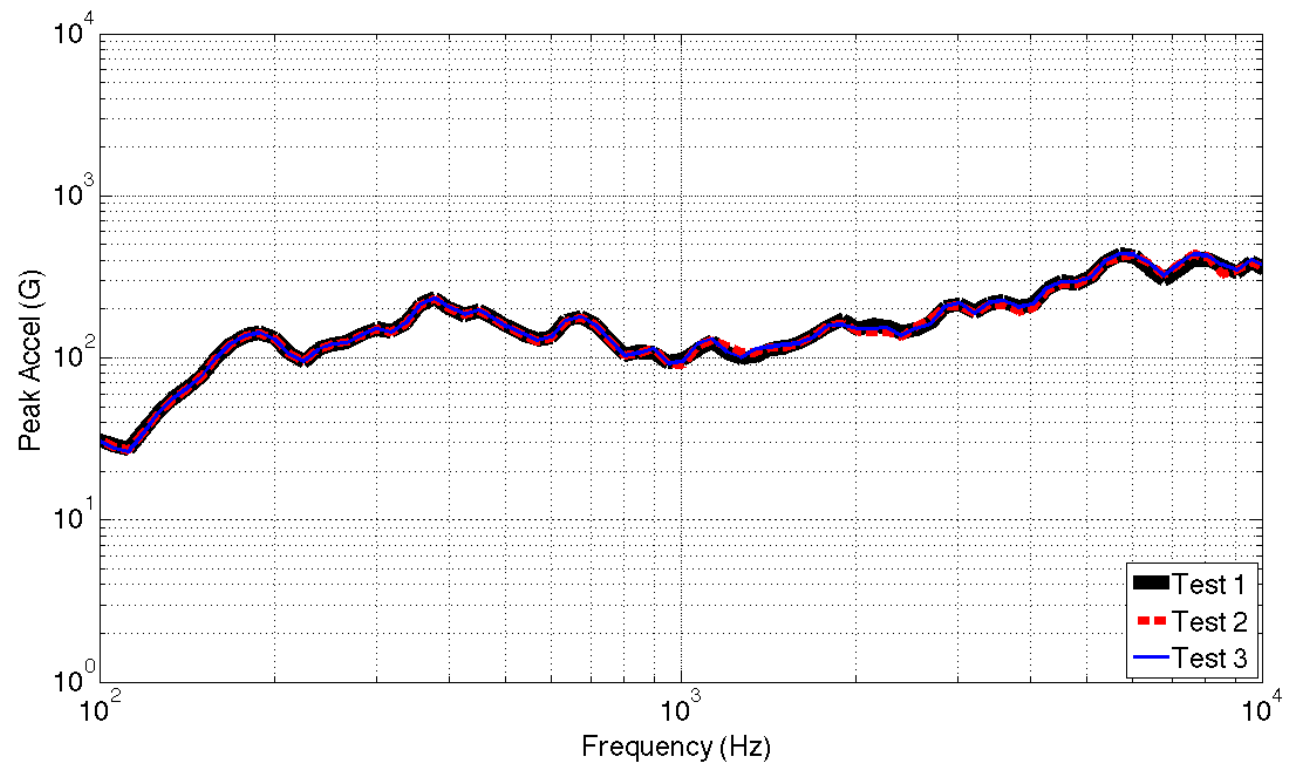

Figure 3.2 Repeatability test.

The three curves are nearly identical, with a maximum difference between the three of about $0.86 \mathrm{~dB}$. This easily meets the less than $1 \mathrm{~dB}$ difference necessary for repeatability and should give confidence that any changes seen in the following tests are in fact due to the chosen parameter.

\subsection{Release Angle}

As the name implies, release angle is the angle from which the pendulum arm is dropped. The arm could be dropped anywhere from 0 degrees (hanging straight down) to 180 degrees (sticking straight up) but as mentioned before this introduces repeatability issues as it is difficult to pull the arm to the exact same angle without some sort of assistance. Two sets of the repeatability plates shown in Figure 2.7 were created. When the new hammer was designed the goal was to make it as small as possible in order to emphasize the effect of adding weight. In doing so issues with the pendulum frame being too close to the shock table frame were introduced, and the number of impact locations was limited as a result. This was resolved by rotating the pendulum arm from the side of 
the pivot point opposite the shock table apparatus to the same side as the apparatus. Some testing with one set of repeatability plates had already been done prior to this rotation, so a new set had to be made. Both sets were nominally supposed to stop the arm at 10,15 , $20,25,30,33,36,39,42,45,48,51,54,57,60,65,70,75$, and 80 degrees, with the first set having an additional stop at 85 degrees. Due to presumably manufacturing errors, neither set of plates matched the nominal values exactly, with the actual angles being 1-2 degrees higher. This is not an issue since the repeatability plates' purpose was to provide a consistent stop for the pendulum arm at a known angle, and they succeed in that.

\subsubsection{Out of Plane}

Changing the release angle should not result in a significant change to the shape of the SRS. It should shift the entire curve up by about the same amount across all frequencies. This parameter is important as tuning it is the easiest way to approximately match the test SRS's magnitude to that of the required SRS.

These tests were run with the first set of repeatability plates and those associated angles, and with no extra mass attached to the hammer. Figure 3.3 shows the SRS curves corresponding to release angles of $12.9,31.5$, and 46.0 degrees.

The curves exhibit a very similar overall shape, as expected. The tests with a higher release angle seemed to excite a few more modes in the shock table apparatus, as evidenced by the additional peaks in the lower frequency portion of the 31.5 and 46.0 degree curves. One interesting result is the conversion of the valley around $1000 \mathrm{~Hz}$ in the 12.9 degree curve to peaks in the 31.5 and 46.0 degree curves. Again, this is likely due to the excitation of one of the apparatus's modes, but such a drastic change should be

noted when conducting further testing. This mode could be difficult to attenuate if one of 
those peaks becomes an over-test for a given SRS requirement. Another interesting note from this test is the minor divergence of the curves as the frequencies increase. It was hypothesized that an increase in release angle would result in a uniform increase of the curve across all frequencies. However, the 31.5 and 46.0 degree curves are clearly further apart around $5000 \mathrm{~Hz}$ than $500 \mathrm{~Hz}$. This will become a theme of the testing going forward: as the release angle increases the SRS curve rapidly increases in the upper frequencies. All in all the results are encouraging as they show that modifying the release angle does give control over the amplitude of the SRS.

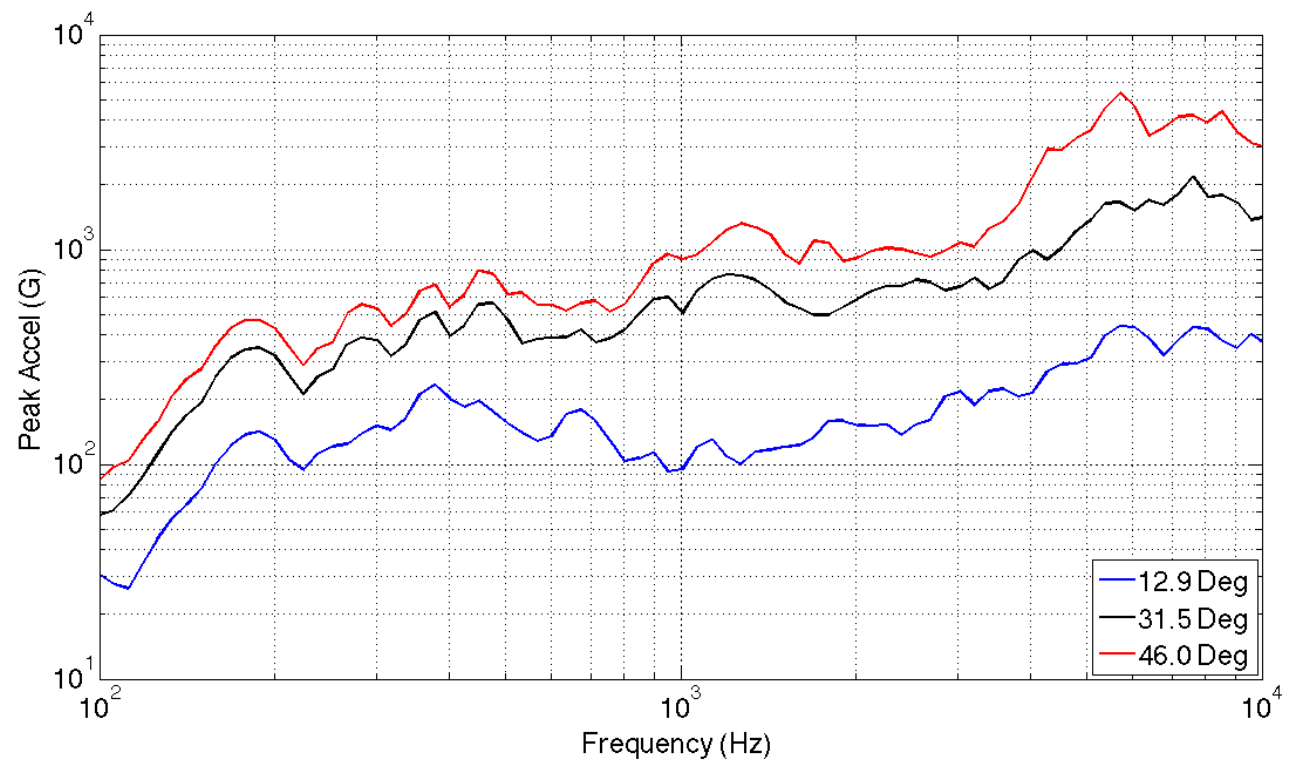

Figure 3.3 Out of plane release angle test.

\subsubsection{In Plane}

The effect here was expected to be the same as for the out of plane testing. An increase in release angle should globally shift the SRS up by the same amount across all frequencies. Ideally all of the parameters discussed in this chapter will display the same characteristics for in plane impacts as they do for out of plane impacts. 
These tests were also done with the first set of repeatability plates and with no extra mass attached to the hammer. Figure 3.4 shows the results of release angles of 12.9, 46.0, and 83.5 degrees.

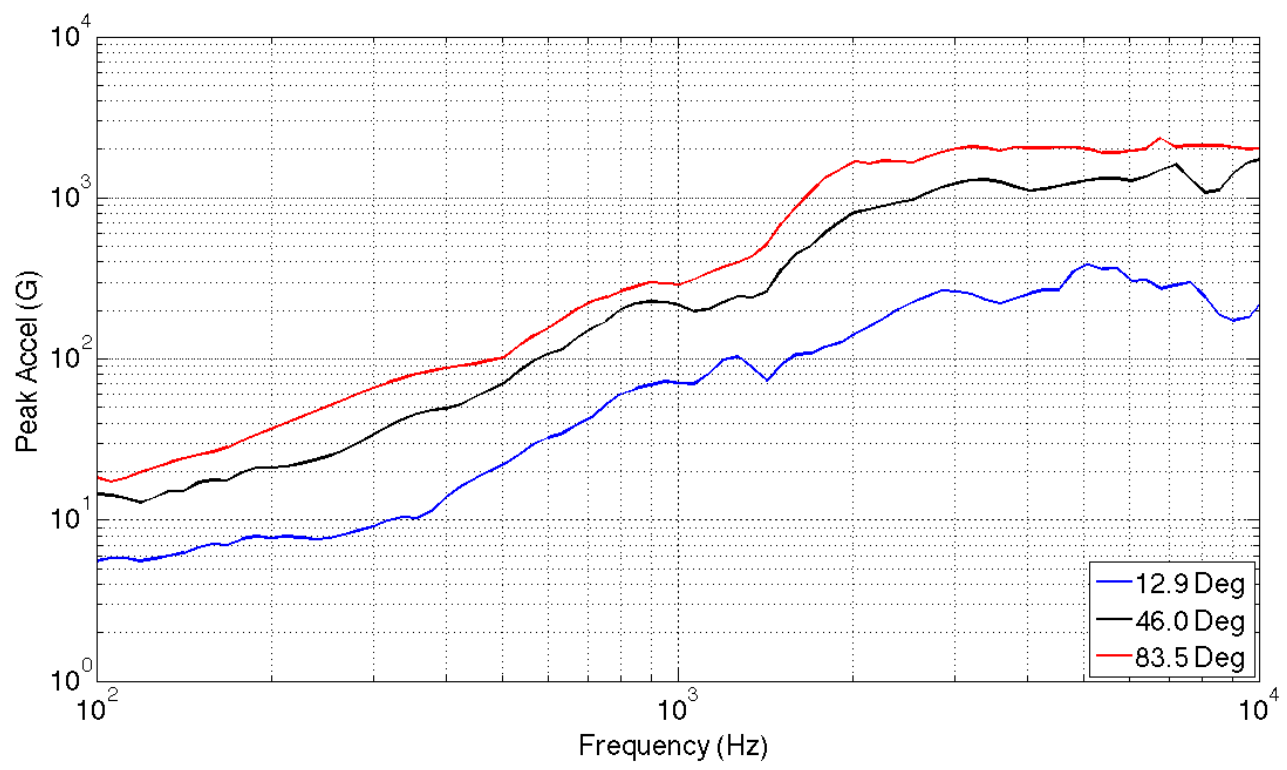

Figure 3.4 In plane release angle test.

These curves also exhibit a similar shape, as expected. Note that between 1000 and $2000 \mathrm{~Hz}$ the two higher release angles exhibit a larger slope than the lower release angle. Again, this is likely due to some mode that becomes more dominant as the release angle increases. The in plane testing presented a more uniform global shift than did the out of plane testing. There is still a bit of divergence between the 12.9 and 46.0 degree curves in the very upper frequencies, but it is not nearly as pronounced or over as wide a range of frequencies as in the out of plane test. A very important difference was noticed between the in plane and out of plane curve shapes. The in plane curves have a larger positive slope in the lower frequencies that is characteristic of a pyroshock curve. This results in a lower amplitude in the lower frequencies and a higher amplitude in the higher frequencies than the out of plane curves. This general shape is fundamental to the shock 
table apparatus: it is a characteristic of the setup. This can be a positive result in that it gives the apparatus the ability to match two different requirement SRS shapes, but it can also be a negative result in that it will be difficult to match the same SRS shape for both out of plane and in plane impacts in order to qualify a test piece in all three axes. This issue will be discussed further in section 3.11. Also note that the release angle varied from 12.9 to 46.0 degrees for the out of plane test and from 12.9 to 83.5 degrees for the in plane test. It was found that the effect the release angle had on the curve amplitude was significantly less for the in plane impacts than for the out of plane impacts. Unfortunately, this means that the in plane impacts cannot create as varied a set of SRS curves as can the out of plane impacts.

\subsection{Hammer Mass}

As described in section 2.3.3, a new hammer was designed to allow for changing hammer mass. The hammer has an unloaded mass of a little over $3 \mathrm{~kg}$. Four weights were machined to allow for several combinations of extra mass. The goal was to make weights of $1,2,3$, and $4 \mathrm{~kg}$ so that any integer between 1 and $10 \mathrm{~kg}$ could be added. Size constraints for the weights resulted in slightly smaller masses, but the desired mass pattern was achieved so that ten combinations of weights could be created. The final weights had masses of $0.9,1.8,2.7$, and $3.6 \mathrm{~kg}$. This allowed for any amount of mass from 0 to $9 \mathrm{~kg}$ to be added in $0.9 \mathrm{~kg}$ steps.

\subsubsection{Out of Plane}

Based on results described in the literature [30], increasing the hammer mass was expected to increase the amplitude of the SRS below about $1000 \mathrm{~Hz}$. This would be a useful result as it would give some control of the curve's slope in the lower frequencies. 
These tests were done with the first set of repeatability plates and a release angle of 46.0 degrees. One impact was done with no extra mass and then impacts with 4.5 extra $\mathrm{kg}$ and 9.0 extra $\mathrm{kg}$ were tested. The results can be seen in Figure 3.5.

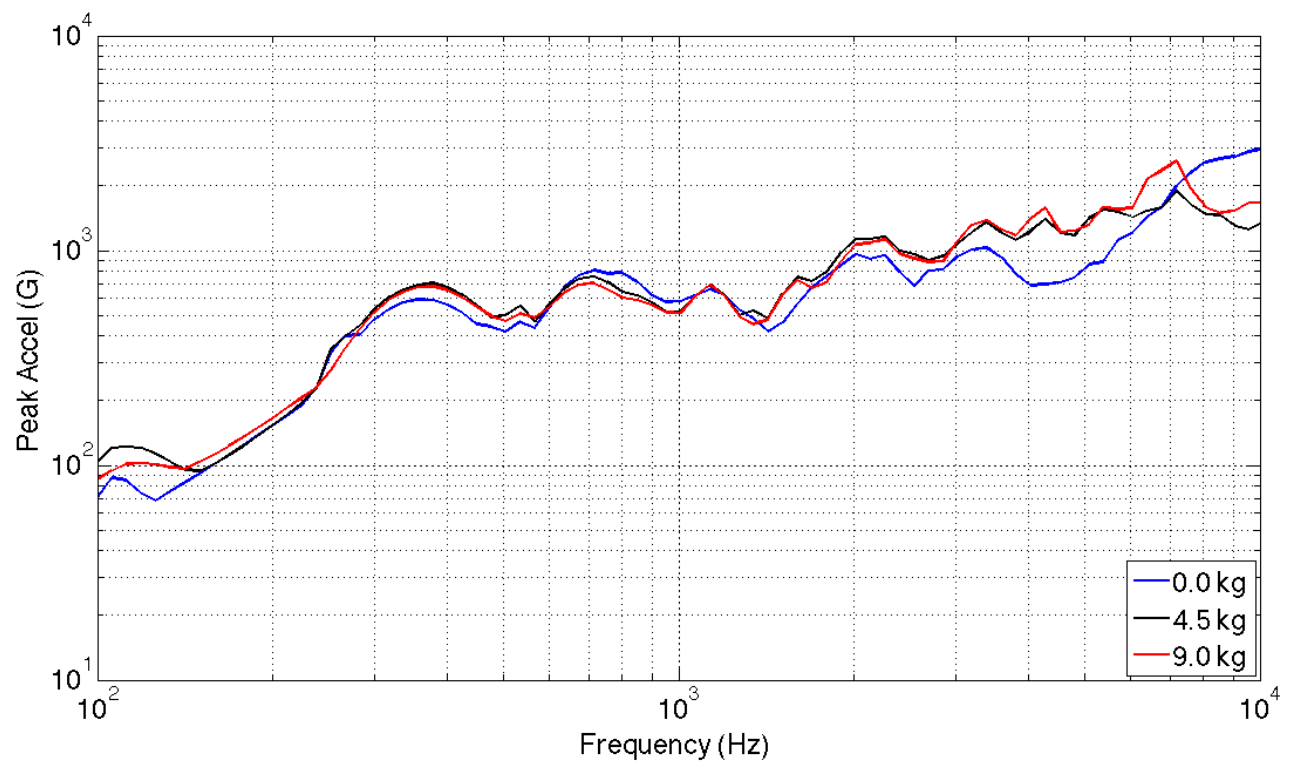

Figure 3.5 Out of plane hammer mass test.

This test's results were disappointing. There was very little difference between the curves from 100 to $1000 \mathrm{~Hz}$. The only noticeable difference in this range was from 100 to $200 \mathrm{~Hz}$, but this did not follow a predictable trend as the $4.5 \mathrm{~kg}$ mass increased that part of the curve more than the $9.0 \mathrm{~kg}$ mass. Additionally, from about 2000 to $7000 \mathrm{~Hz}$ the 4.5 and $9.0 \mathrm{~kg}$ mass curves were very similar and higher in amplitude than the no extra mass curve. It is unknown to the author why these results differ from the literature so greatly, but one possible reason is the nature of the apparatus. Perhaps the aluminum plate being suspended vertically instead of horizontally affects the part of the plate's response that is most susceptible to the changing hammer mass. The author did not want to abandon this parameter as the ability to manipulate the lower frequencies of the curve would be important for running actual tests. Therefore, adding extra mass was again tried while 
trying to match a requirement SRS with a test piece mounted to the plate. As can be seen in Figure 3.6, these results were more in line with expectations. The test with $9.0 \mathrm{~kg}$ of extra mass was noticeably higher in amplitude than the test with no extra mass from 100 to $300 \mathrm{~Hz}$, and virtually identical for the rest of the frequencies. The effect was not as dramatic and did not affect as many frequencies as desired, but it would prove important in tuning the SRS to match the requirement.

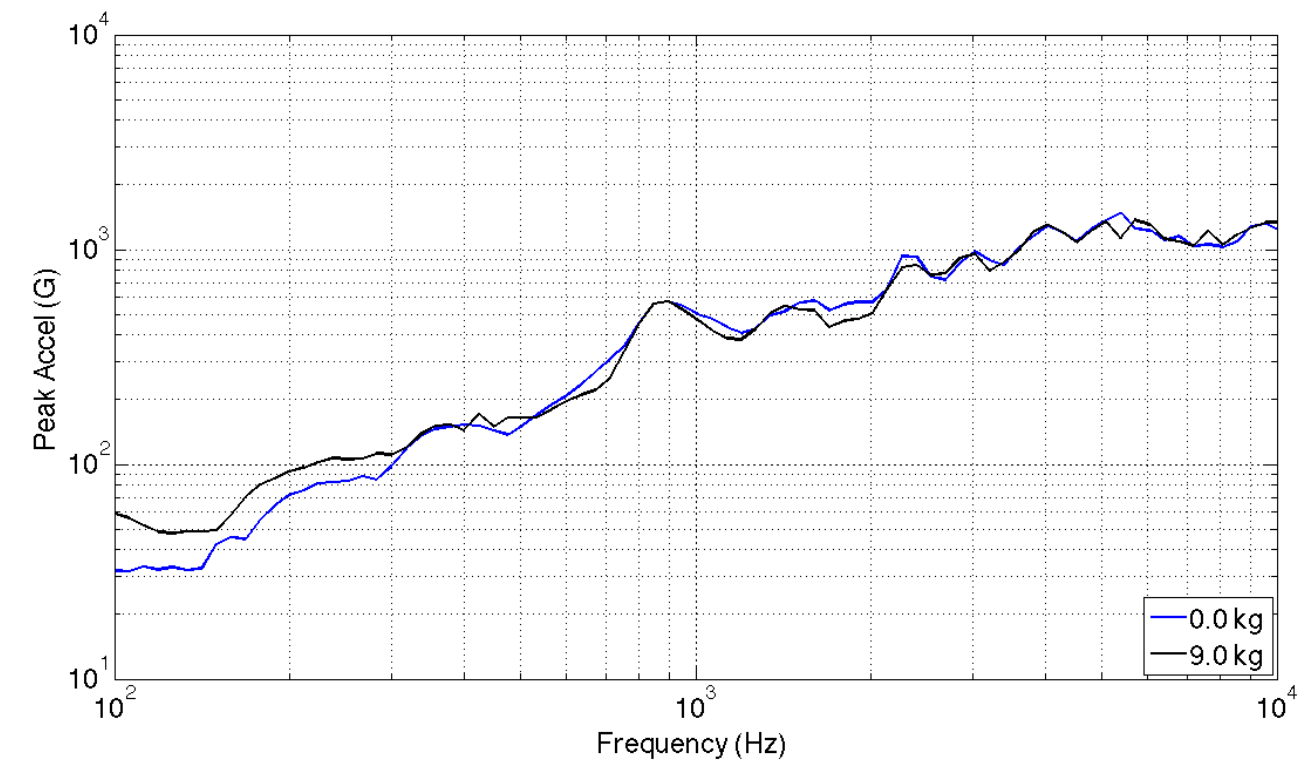

Figure 3.6 Out of plane hammer mass test with mounted test piece.

\subsubsection{In Plane}

With an in plane impact strike there should be an increase in the lower frequencies of the SRS with a mass increase. This would be particularly beneficial for this apparatus as the lower frequencies start at an extremely low amplitude. Having the ability to increase the amplitude of those frequencies without increasing the already high amplitude of the higher frequencies would greatly increase the variety of SRS curves that could be generated. However, the effect of adding mass could be smaller for an in plane strike compared to an out of plane strike, based on the results of the release angle tests. 
The in plane hammer mass tests were conducted with the first set of repeatability plates and a release angle of 51.7 degrees. One impact was done with no extra mass and the other impact had $4.5 \mathrm{~kg}$ of extra mass attached. Figure 3.7 presents the results.

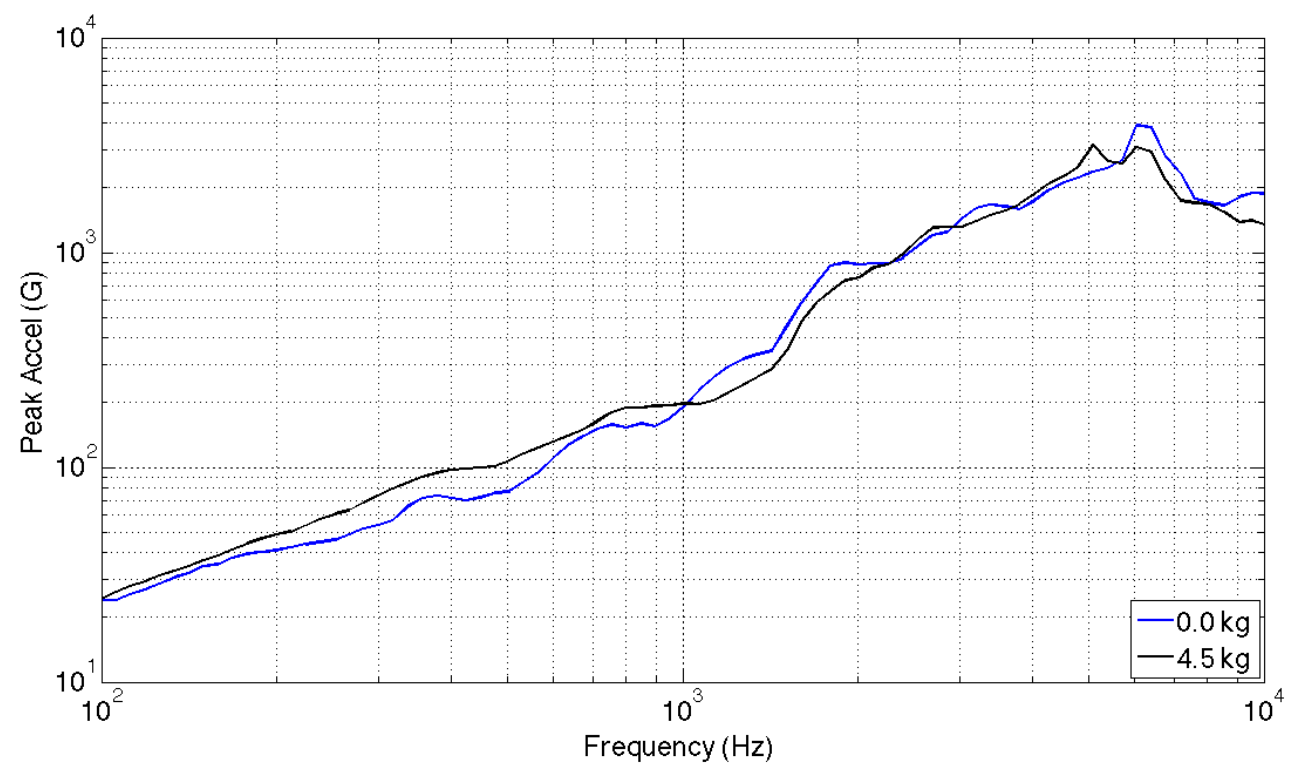

Figure 3.7 In plane hammer mass test.

The two curves are very similar up to almost $200 \mathrm{~Hz}$. From there until $1000 \mathrm{~Hz}$ the extra mass did indeed increase the curve's amplitude. Oddly enough, the extra mass resulted in a lower amplitude from about 1000 to $2000 \mathrm{~Hz}$, after which point the two curves agreed well. At a high level this is a desirable result, as it simultaneously increase the lower amplitude portion of the curve and decreases a part of the higher amplitude portion of the curve. As the author feared, however, the effect was rather small. The largest difference was less than $3 \mathrm{~dB}$ and the average difference from 100 to $1000 \mathrm{~Hz}$ was only $1.59 \mathrm{~dB}$, which is about $20 \%$. Common tolerances given with a requirement SRS range from 3 to $9 \mathrm{~dB}$, so clearly $1.59 \mathrm{~dB}$ is not a very significant difference. Therefore, unless a test SRS is already within or very close to meeting a given tolerance, increasing the mass is unlikely to be of much help for an in plane strike. 


\subsection{Hammer Head Diameter}

In this paper, hammer refers to the block of metal at the end of the pendulum arm. This block can have mass added to it via a threaded rod, and it has a hole in the side that faces the aluminum plate when the arm is hanging straight down. The hammer head goes in this hole and is what actually impacts the aluminum plate. The hole has a 1-inch diameter and the hammer heads are held in place with a set screw. The hammer head used for the majority of the testing was simply a cylinder with a slightly smaller diameter (0.005 in smaller) than the hole. Two more hammer heads were made however. Both were the same almost 1-inch diameter along about half their lengths so that they could be secured in the same hole. One of them expanded out to a 2-inch diameter for the rest of the length and the other contracted to a 0.5 -inch diameter.

\subsubsection{Out of Plane}

Other shock test facilities [30] have reported an increase in the SRS above 2000 $\mathrm{Hz}$ with an increase in hammer head radius. This would be an especially useful parameter for in plane impacts if it performed as expected, as it would allow the user to attenuate the high amplitude response in the higher frequencies.

The first set of repeatability plates were used in these tests with a release angle of 46.0 degrees. Each hammer head was used, allowing diameters of 0.5 -inch, 1-inch, and 2inches to be tested. Figure 3.8 shows the results.

The lower frequency part of the graph is nearly perfect. From 100 to about 1200 $\mathrm{Hz}$ the three curves are nearly identical. Beyond that frequency the behavior becomes a bit unruly. From 1600 to about $4000 \mathrm{~Hz}$ the SRS amplitude decreases as the hammer head diameter increases. For approximately the next $4000 \mathrm{~Hz}$ that rule reverses and 
follows the expected trend of increasing amplitude with increasing hammer head diameter. The last $2000 \mathrm{~Hz}$ does not seem to exhibit much of a pattern at all. Unfortunately these trends did not strike the author as useful due to their inconsistency and small magnitude. As will be discussed later, a more effective method of decreasing the curve's amplitude in the higher frequencies was found. If a user needed to increase the amplitude of the high frequencies, the 2-inch diameter hammer head could be tried, but it seemed to excite a mode around $7000 \mathrm{~Hz}$ that would likely result in an over-test. For these reasons, testing with this parameter was not conducted for in plane strikes.

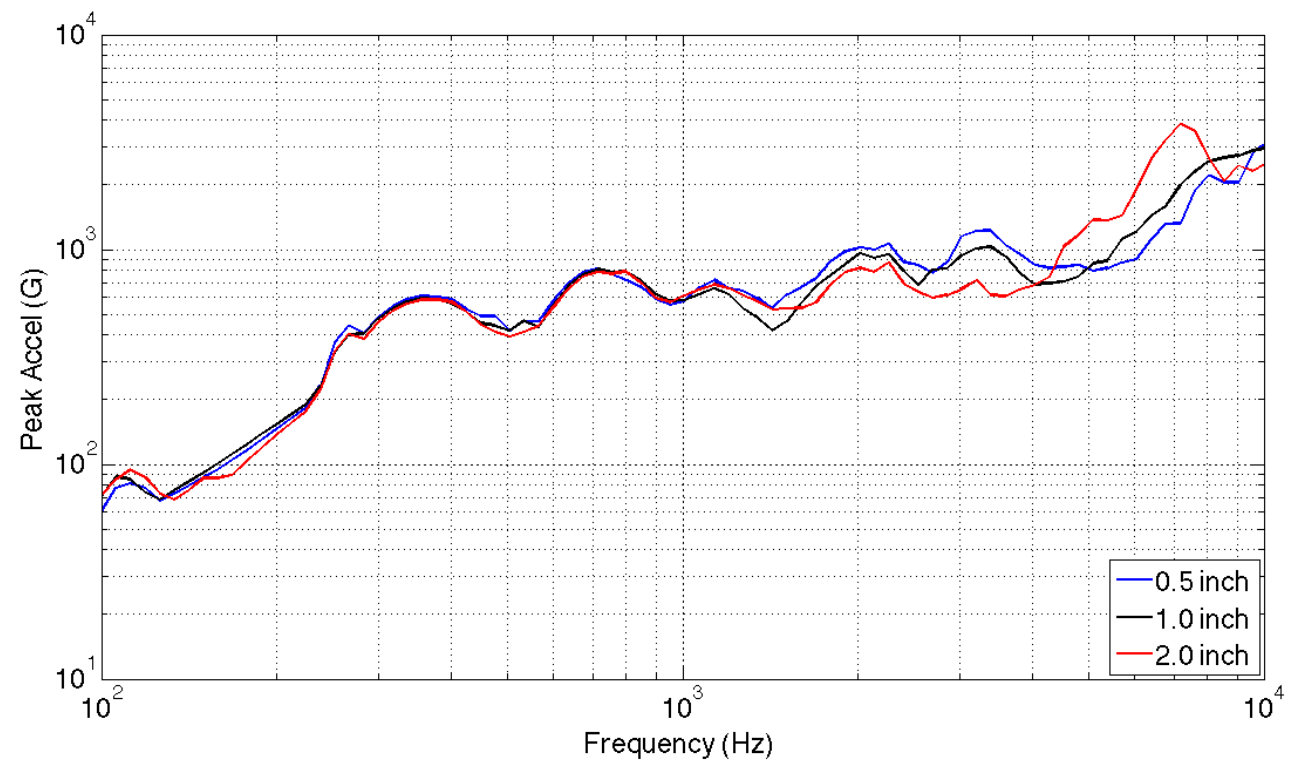

Figure 3.8 Out of plane hammer head diameter test.

\subsection{Hammer Head Material}

The original hammer inspired the design of the new hammer, and thus also had an approximately 1 -inch diameter hole with a set screw to hold the hammer head in place. The group of students that assembled the original hammer chose titanium as the material for the hammer head. The three hammer heads created for this project were all made of steel. 


\subsubsection{Out of Plane}

The author's expectations were that a harder material would result in higher amplitudes in the higher frequencies because the contact between the hammer head and the plate would occur over a shorter amount of time. If this proved to be true, a softer material could be used as a hammer head in an attempt to help with excessively high amplitudes in the affected frequency range.

The first set of repeatability plates and a release angle of 46.0 degrees were again used. Figure 3.9 shows the results for both the titanium and steel hammer heads, with all other parameters held constant.

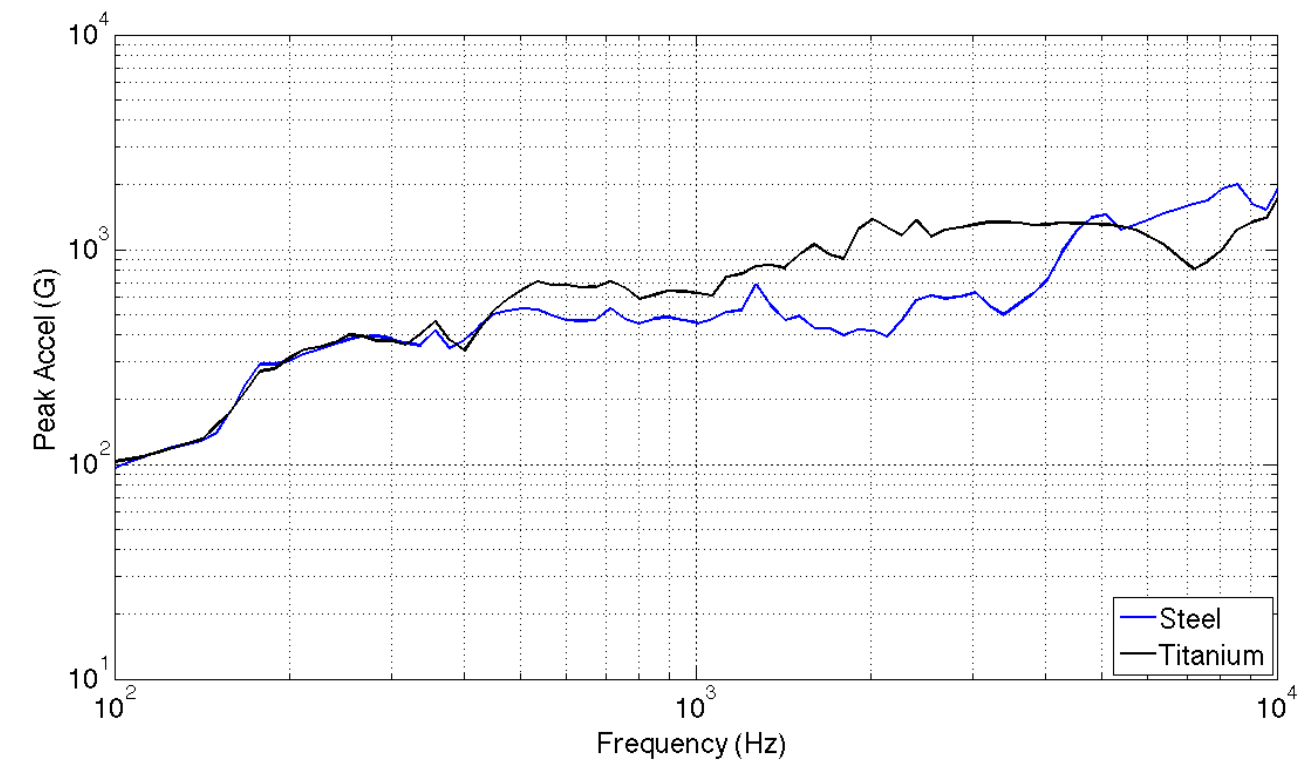

Figure 3.9 Out of plane hammer head material test.

The two curves are virtually identical from 100 to about $450 \mathrm{~Hz}$. From $450 \mathrm{~Hz}$ to about $5000 \mathrm{~Hz}$ the harder titanium hammer head resulted in higher amplitudes as hypothesized. However, as happened with the hammer head radius test the trend flipped for the very highest frequencies. A positive attribute of this test is the magnitude of the difference between the two curves. Over the affected frequency range the average 
difference is $4.2 \mathrm{~dB}$ with a maximum of over $10 \mathrm{~dB}$. If a user had a requirement SRS with very low amplitudes in the high frequencies, manufacturing a hammer head out of aluminum or a hard plastic might be a good starting point.

\subsection{Mechanical Filter at Impact}

Mechanical filter here is perhaps a euphemism; it really refers to any material placed between the hammer head and the aluminum plate that changes the dynamics and timing of the impact. This material could be as complex as high density foam and rosewood or as simple as paper and cardboard. It should be noted that none of the tests run actually involved a direct impact between the hammer head and the aluminum plate. Impacts between a steel or titanium hammer head and an aluminum plate would eventually lead to permanent deformation of and damage to the aluminum plate, which would likely necessitate its replacement. The plate's large (4-foot by 6-foot) size makes its replacement too expensive (likely close to $\$ 1000$ ) to be reasonable. Therefore, a small sacrificial plate is used to bear the brunt of the potential damage caused by an impact while still transferring a large amount of energy to the aluminum plate. The approximately 4-inch by 7-inch steel sacrificial plate bolts securely to the aluminum plate and is small enough to be cost-effective when replacement is necessary. It also presents another parameter that could be varied: sacrificial plate material, although the author did not pursue that opportunity. The mechanical filter could be placed either between the sacrificial plate and the aluminum plate or between the sacrificial plate and the hammer head. If the filter were placed in the former position, care would need to be exercised if the mechanical filter material could easily deform significantly more than the steel sacrificial plate. A thick filter of this material could result in permanent damage to the 
sacrificial plate due to the filter not rigidly supporting the impacted portion of the plate. The author did not want to worry about this possibility, so the mechanical filters were placed between the sacrificial plate and the hammer head.

\subsubsection{Out of Plane}

Since the mechanical filter essentially served the same purpose as using a softer material for a hammer head, the behavior was expected to be similar. The use of a mechanical filter would attenuate the SRS amplitude in the higher frequencies, and a thicker filter would enhance this trend. This would be useful and practical as it is much easier to attach a piece of cardboard, for example, to the sacrificial plate than it is to machine a new hammer head.

This test used the second set of repeatability plates as the pendulum arm position had been changed. The release angle was 42.9 degrees and cardboard was the mechanical filter material of choice due to its ready availability and readiness to deform. Note that since the material does deform so easily, a new piece should be used for each run to achieve consistent results. If a user wishes to avoid extensive material replacement, a rubber material is suggested. Tests were performed with no mechanical filter and with 1/8-inch and 1/4-inch thick cardboard, as shown in Figure 3.10.

Other than a small deviation from about 100 to $150 \mathrm{~Hz}$, the three curves are nearly identical up to $1500 \mathrm{~Hz}$. From there the mechanical filters attenuate the SRS amplitude in the higher frequencies as expected. An interesting feature is the similarity between the 1/8-inch and 1/4-inch cardboard curves. The thicker filter actually attenuated slightly less than the thinner filter did. While this could be an indication of thicker filters doing less, the author believes it is more likely a product of two $1 / 8$-inch pieces of cardboard being 
used at the same time. The author believes that a single piece of thicker or thinner cardboard would further support the hypothesis, but all of the available cardboard was the same thickness. This parameter was deemed to be more useful by the author than the other two parameters that affected the same frequency range: hammer head diameter and hammer head material. The width of the affected frequency range and the lack of a very high frequency trend reversal (which was present in both of the other parameters) led to this conclusion.

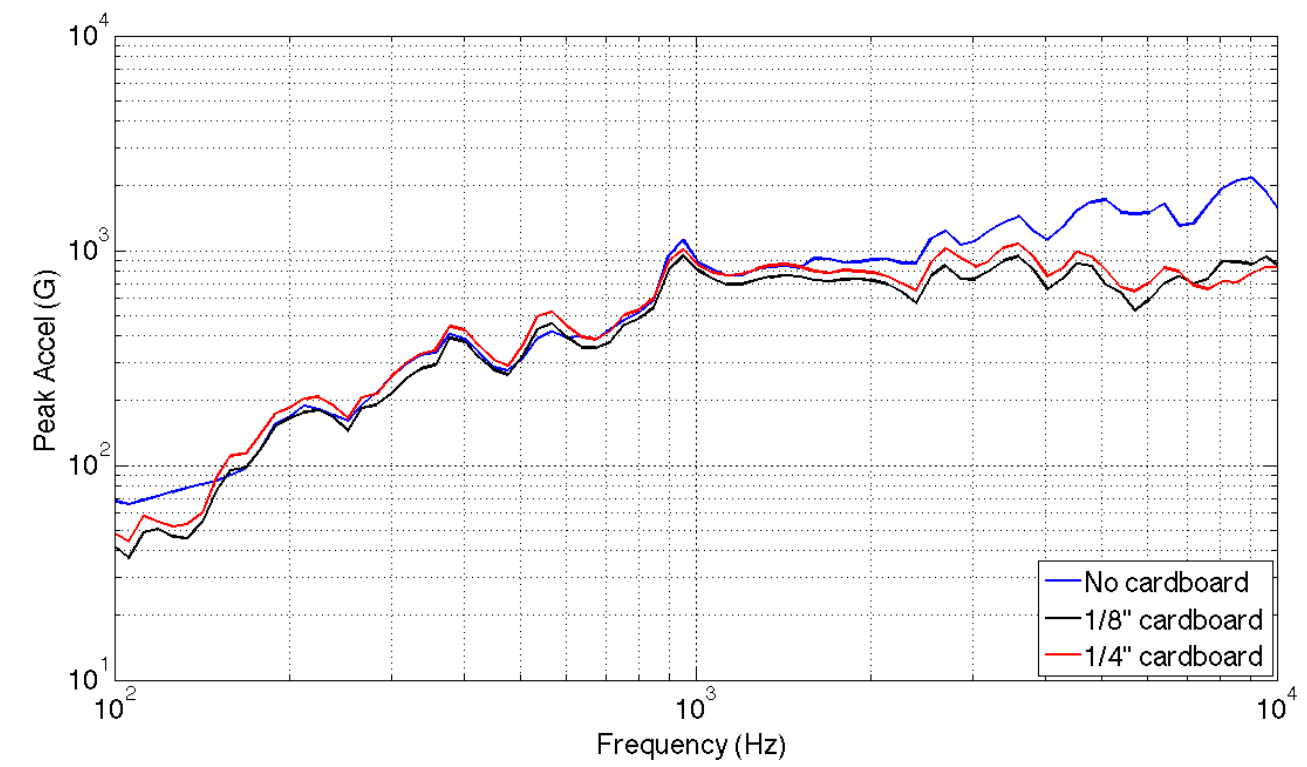

Figure 3.10 Out of plane mechanical filter test.

\subsubsection{In Plane}

The in plane trend was expected to be similar to the out of plane trend. Due to the magnitude of the other parameters' trends being smaller for the in plane impacts compared to the out of plane impacts, a similar phenomenon was expected for this parameter. As discussed in the last two parameters' sections, this behavior would be helpful in attenuating the high frequency, high amplitude portion of the in plane SRS. 
The pendulum arm was released from 52.0 degrees as measured by the second set of repeatability plates. Figure 3.11 displays the results of test with no cardboard as well as with 1/8-inch and 1/4-inch thick pieces of cardboard.

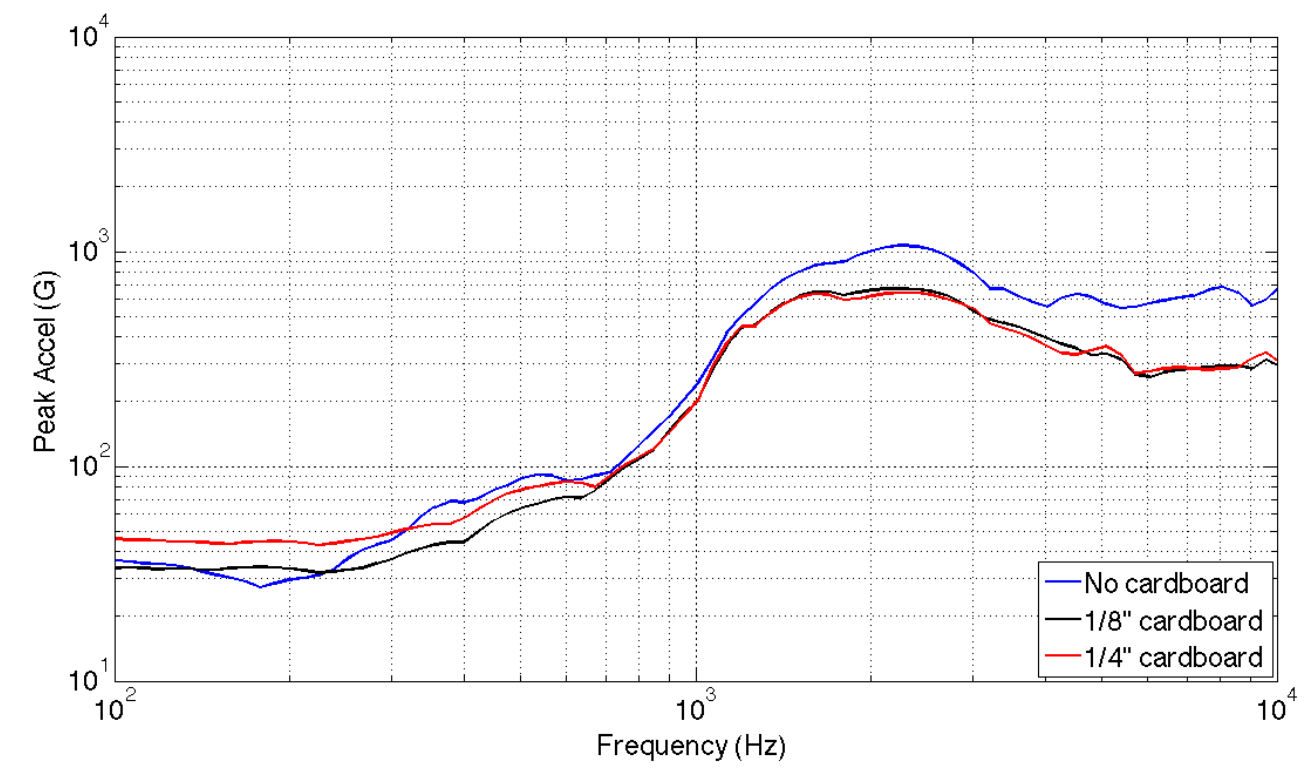

Figure 3.11 In plane mechanical filter test.

The results were as expected from about $700 \mathrm{~Hz}$ to the high frequency portion of the graph. The curves were nearly identical from 700 to $1200 \mathrm{~Hz}$, and the filters again attenuated all frequencies above that. Surprisingly, the level of attenuation was comparable to that seen in the out of plane tests. Perhaps this suggests that the amount of attenuation done by a mechanical filter depends more on the original type of impact (materials involved) than the shape of the original SRS. This test resulted in virtually the exact same amount of attenuation by the two filter thicknesses. Again, the author believes this is due to the 1/4-inch cardboard filter being two layers instead of one continuous piece. Below $700 \mathrm{~Hz}$ the curves are noticeably different and don't display the positive slope that is characteristic of the in plane SRS. This is likely due to issues that arose with the in plane testing as discussed in section 3.11, not some aspect of the filter. 


\subsection{Sensor Location}

The 48-inch by 72 -inch aluminum plate has 77 holes in a 6-inch square pattern: 7 rows and 11 columns. This gives 77 possible locations to attach a sensor. The holes are 1/4-20 threaded through holes, while the accelerometers have a 1/4-28 mounting stud. This required the use of a mounting block to attach the accelerometers to the aluminum plate. To avoid potentially introducing a structural mode to the system, the aluminum mounting block cube was kept less than one inch on each side. [31] For a given sensor location three measurements can be made; one with an accelerometer aligned with the impact direction and two others to make an orthogonal set of axes. If all three axes are measured for a single impact it allows the test engineer to attempt to satisfy the given SRS requirement in all three axes at once. This is desirable as all three axes are generally required anyway and it reduces the total number of shock events that the test piece must endure. Meeting the same SRS in all three axes in one impact is difficult to do, but that accelerometer configuration can still be beneficial in that it allows the non-tested axes in a given impact to be monitored for over-testing.

\subsubsection{Out of Plane}

Every shock test apparatus has a different SRS shape based on its geometry and boundary conditions. Therefore, the author did not have much intuition as to how measuring the data at different locations would affect the SRS. The hypothesis was that the shape would not change much as the accelerometers measured different locations, but the overall SRS magnitude would increase in the middle of the aluminum plate and decrease as the accelerometers moved toward the top and bottom of the plate where it 
was secured to the frame. The SRS magnitude should also increase as the sensor location moves closer to the impact location.

This test was conducted with the first set of repeatability plates and the curves shown were generated with a release angle of 46.0 degrees. Figure 3.12 shows the impact location and four sensor locations corresponding to the four curves shown in Figure 3.13. Note that the color of the " $\mathrm{x}$ " marker for the sensor location matches the color of the corresponding SRS curve.

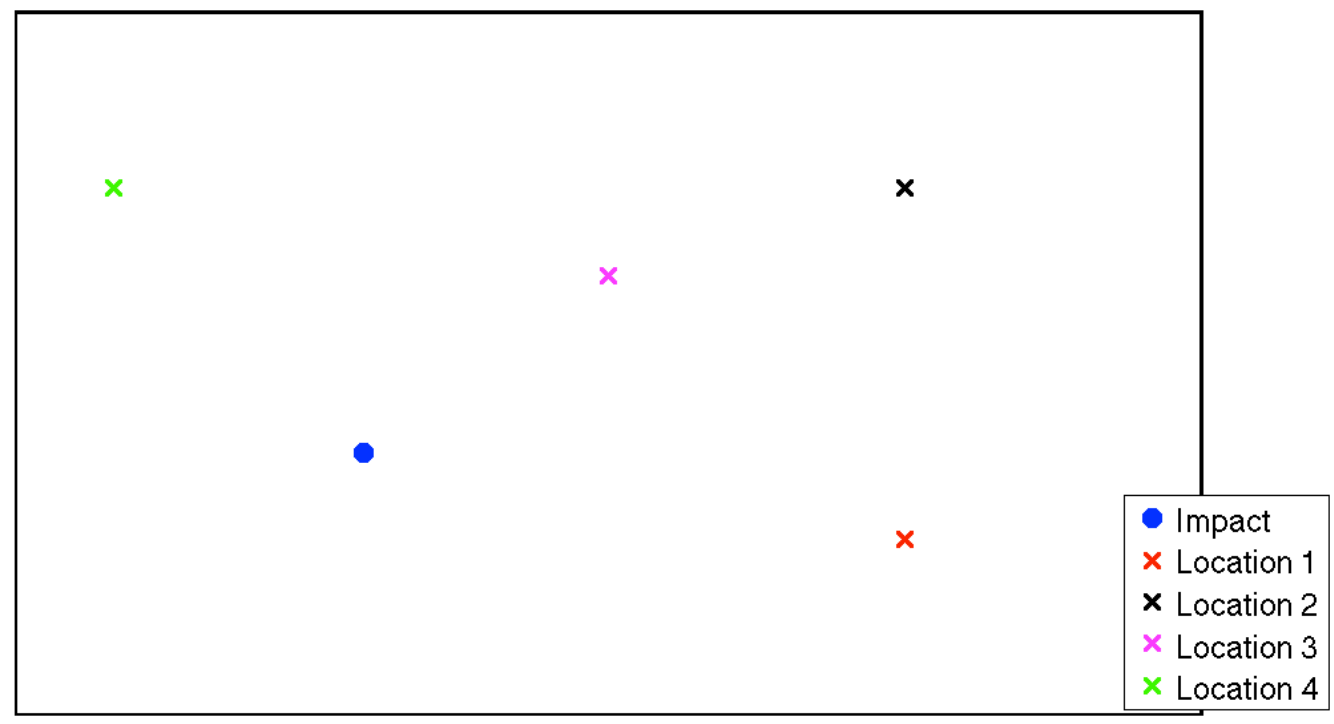

Figure 3.12 Accelerometer locations for out of plane sensor location test.

Locations 1 and 2 resulted in curves of roughly the same magnitude that followed the same basic shape, which was expected as they are the same distance from the edges of the plate. Across most of the frequency range the curve corresponding to location 3 had the largest magnitude, which makes sense as it was closest to the impact location. Location 4 resulted in a curve that was roughly the same magnitude as those corresponding to locations 1 and 2 , but had a shape that more closely followed that of location 3. This was an interesting behavior to notice, and perhaps implies that sensor 
proximity to impact location affects both SRS curve magnitude and shape. The most important conclusion that can be drawn is that moving the sensor closer to the impact location will increase the SRS magnitude.

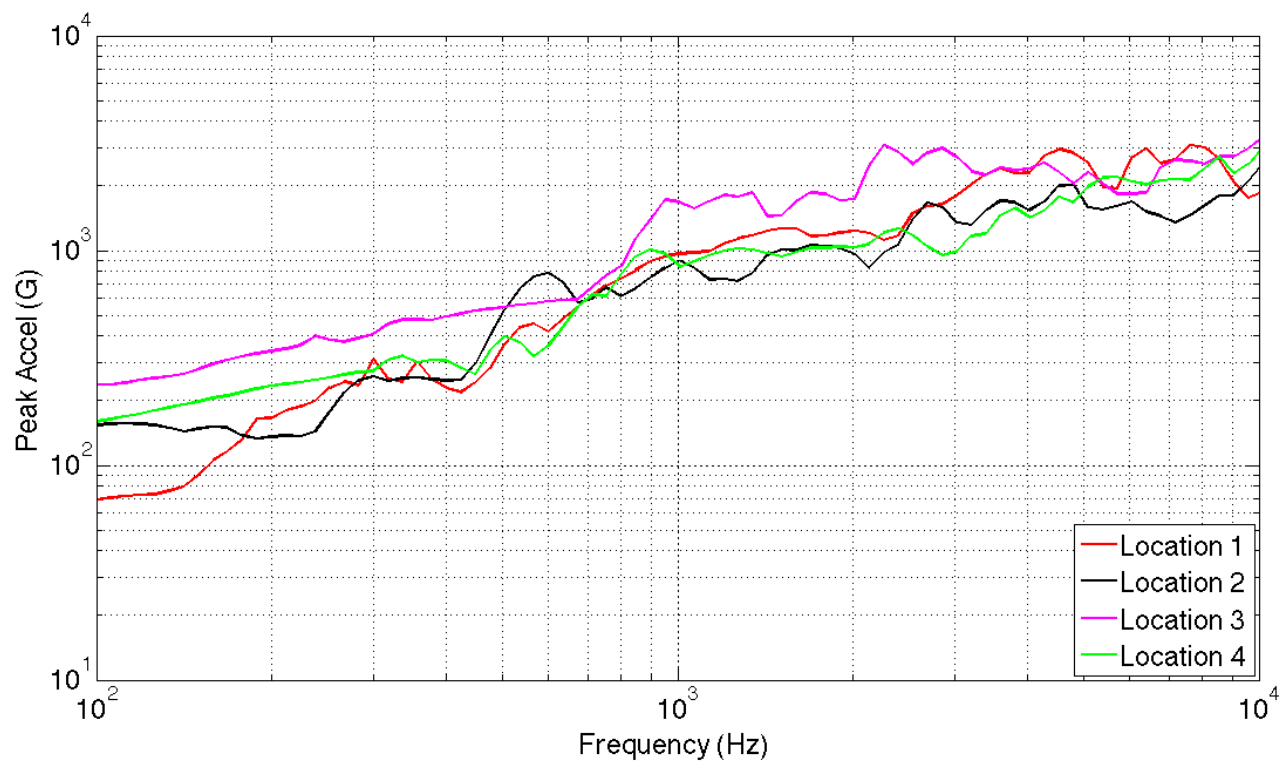

Figure 3.13 Out of plane sensor location test.

\subsubsection{In Plane}

The author expected a similar result from in plane impacts with regards to the effect of moving the accelerometer closer to the edges of the aluminum plate. Moving closer to the part of the plate that is rigidly attached to the frame should reduce the SRS magnitude. However, the effect of changing the distance between the impact and sensor locations was hypothesized to be noticeable smaller for this type of impact.

The test used the first set of repeatability plates and a release angle of 46.0 degrees. The impact location and seven sensor locations are shown in Figure 3.14, with Figure 3.15 depicting the resulting SRS curves. Note that the colors again correspond between the two figures. 


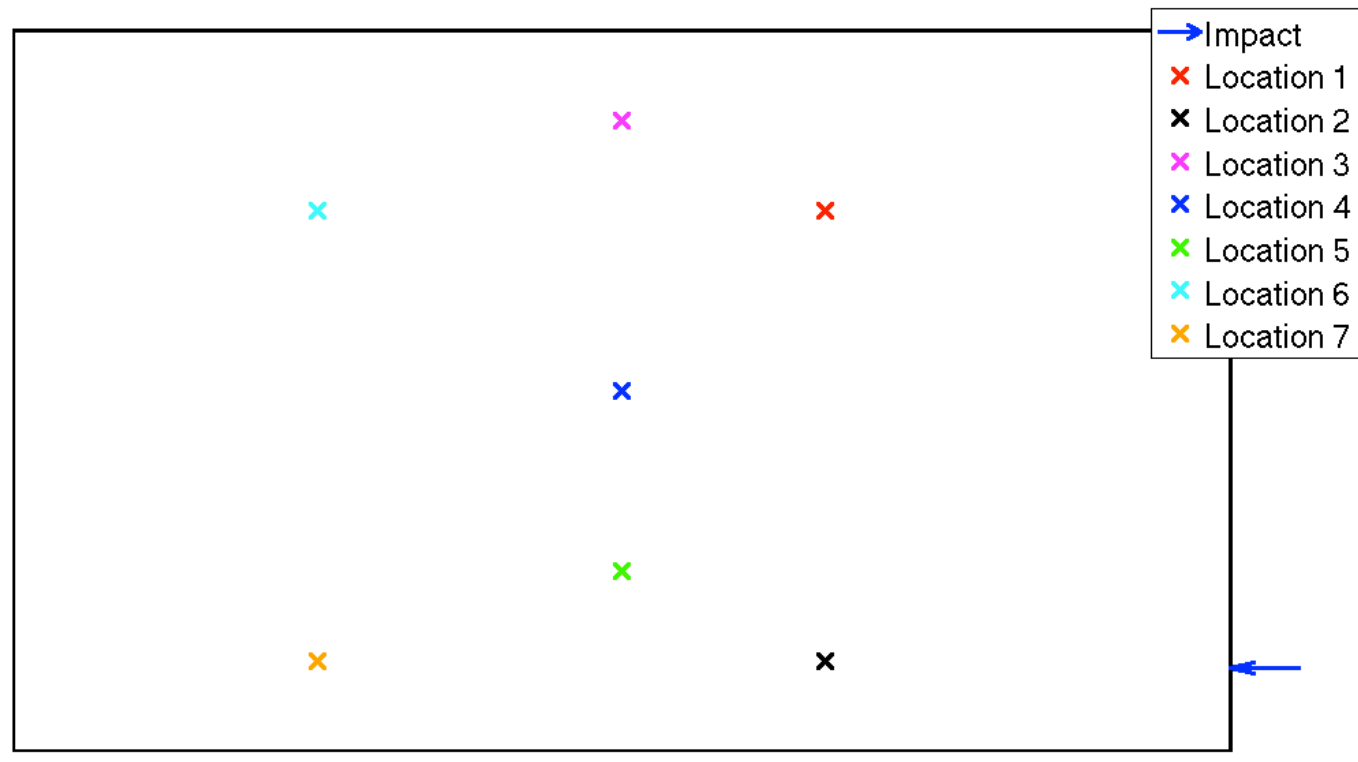

Figure 3.14 Accelerometer locations for in plane sensor location test.

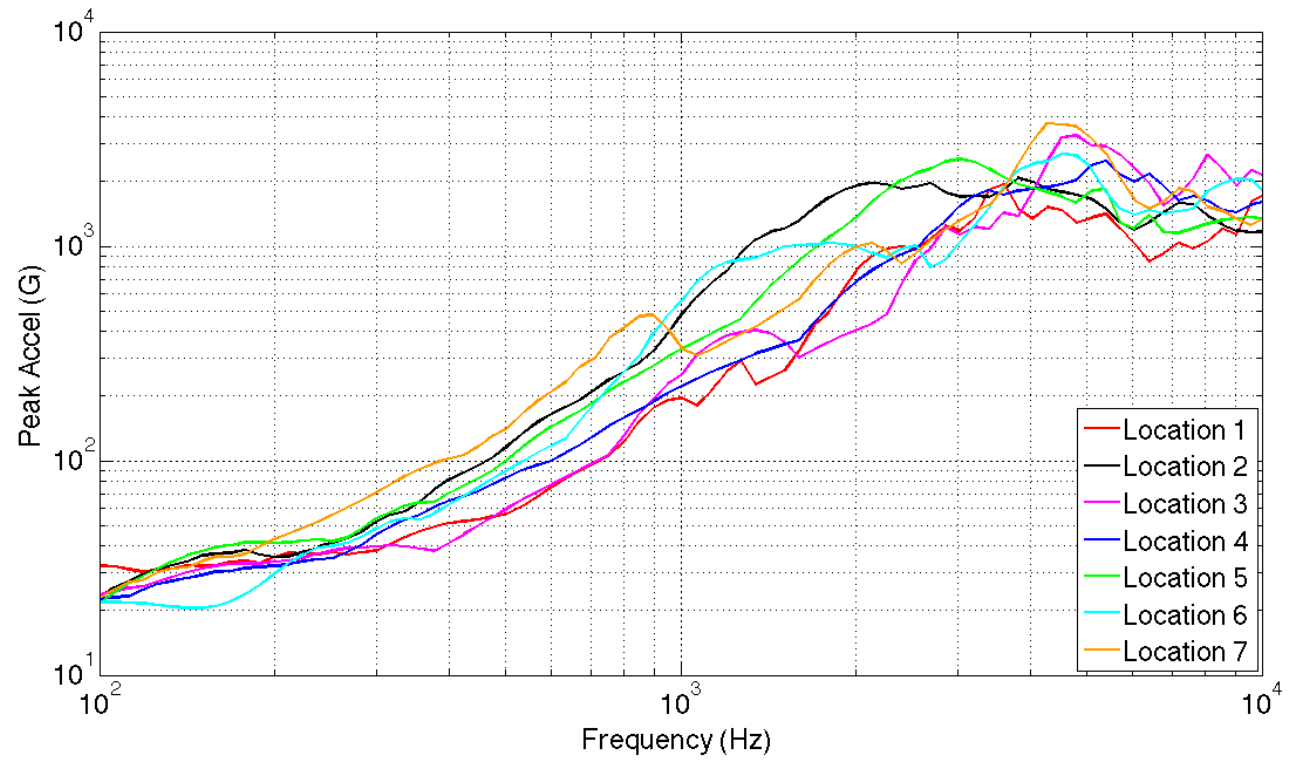

Figure 3.15 In plane sensor location test.

Locations 1 and 2 are the same distance from the impact location horizontally but location 2 is much closer vertically, and this is evident in location 2's larger SRS magnitude. The same trend can be seen in locations 3,4 , and 5 , although the difference is 
less drastic. Interestingly locations 6 and 7 only display that trend until approximately $900 \mathrm{~Hz}$ after which the two curves cross each other several times. The author can conclude that for in plane strikes the SRS magnitude generally increases as the vertical distance between the impact and sensor locations decreases. Locations 1 and 6 are the same vertical distance from the impact location but location 1 is much closer horizontally. Strangely enough the farther away location 6 resulted in a larger SRS magnitude over most of the frequency range. Locations 2 and 7 followed a similar trend except for between 1000 and $3000 \mathrm{~Hz}$. The author concluded that there must be an important mode in the plate's in plane direction that results in movement diagonally from the impact location to the opposite side of the plate.

\subsection{Impact Location}

As discussed in section 3.2, the new hammer was shorter than the old hammer and thus forced the pendulum arm frame to be moved closer to the aluminum plate to ensure that the hammer head was not impacting at an angle. In fact, it had to be moved so close that the pendulum arm frame would hit the fixtures that attached the aluminum plate to its frame, which greatly reduced the possible number of impact locations. This was only an issue in the out of plane configuration. After rotating the pendulum arm, most of the interference points were removed and the impact location could be varied across most of the plate again. Note that the impact locations were still limited to some degree by the use of the sacrificial plate. Since it attached to the plate with bolts through two of the plate's holes, the hammer could not impact directly at a hole. The in plane configuration had much less possible variation in the impact location, as it could only impact a 4-foot line as opposed to the 24-square foot rectangle for the out of plane configuration to impact. 


\subsubsection{Out of Plane}

Impacting the aluminum plate in different locations should excite the various modes by different amounts. Thus, while the overall SRS shape should not change too much, there should be spikes at different frequencies as the impact location is moved. This is likely also dependent on sensor location, but the author chose to only present the results of one sensor location here.

These impacts were performed with the first set of repeatability plates and a release angle of 46.0 degrees. Figure 3.16 shows the sensor location and the four impact locations, while Figure 3.17 presents the SRS curves. The "x" marker color in Figure 3.16 corresponds to the SRS generated by that impact in Figure 3.17.

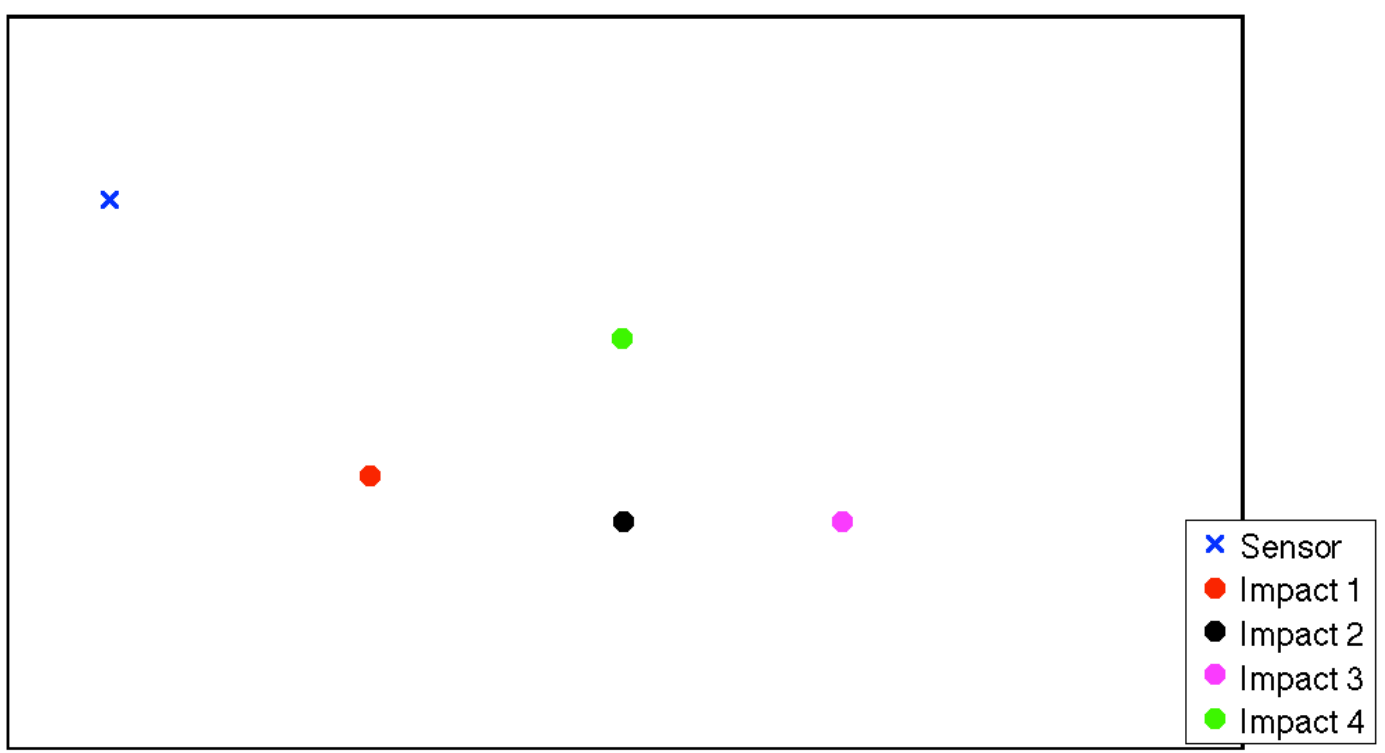

Figure 3.16 Impact locations for the out of plane impact location test.

The hypothesis that changing impact locations would change the location of various spikes in the SRS was clearly correct, as none of the four curves have spikes at the same frequency. The SRS corresponding to impact location 4 has a sharp slope from 
about 200 to $300 \mathrm{~Hz}$ that is absent in the other three curves. This could be a characteristic of the middle horizontal level of the plate. While it does add to the variety of curves that the shock table apparatus can generate, none of the major launch vehicle provider SRS requirements have that trait so it may not be of much use.

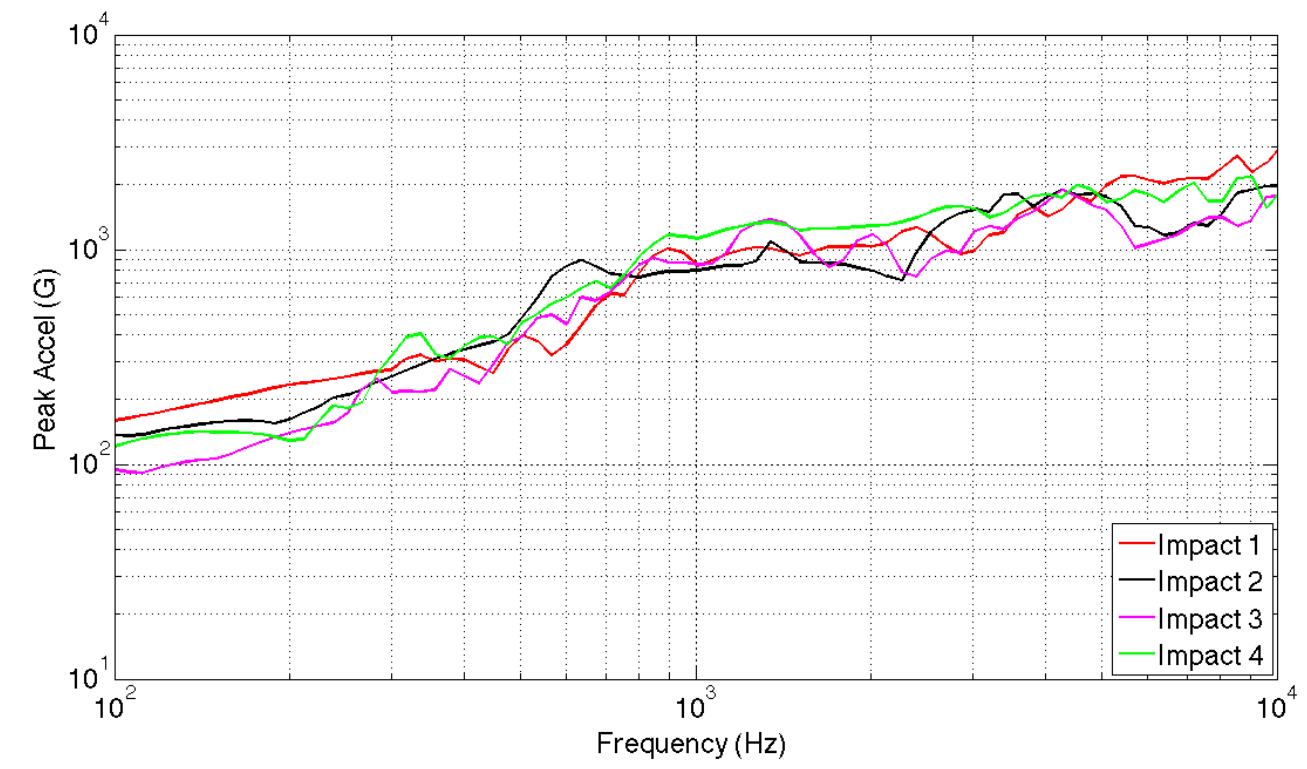

Figure 3.17 Out of plane impact location test.

\subsubsection{In Plane}

The in plane configuration provides fewer options for impact locations. There should be a minor difference between impacts in the middle of the plate's side compared to the bottom, but the author expects them to be less pronounced than the results of the out of plane test.

The release angle was 46.0 degrees as measured by the first set of repeatability plates. Figure 3.18 shows the sensor location and the two impact locations, which correspond to the two SRS curves shown in Figure 3.19. Like colors indicate that the SRS curve was generated from the impact at the given location. 


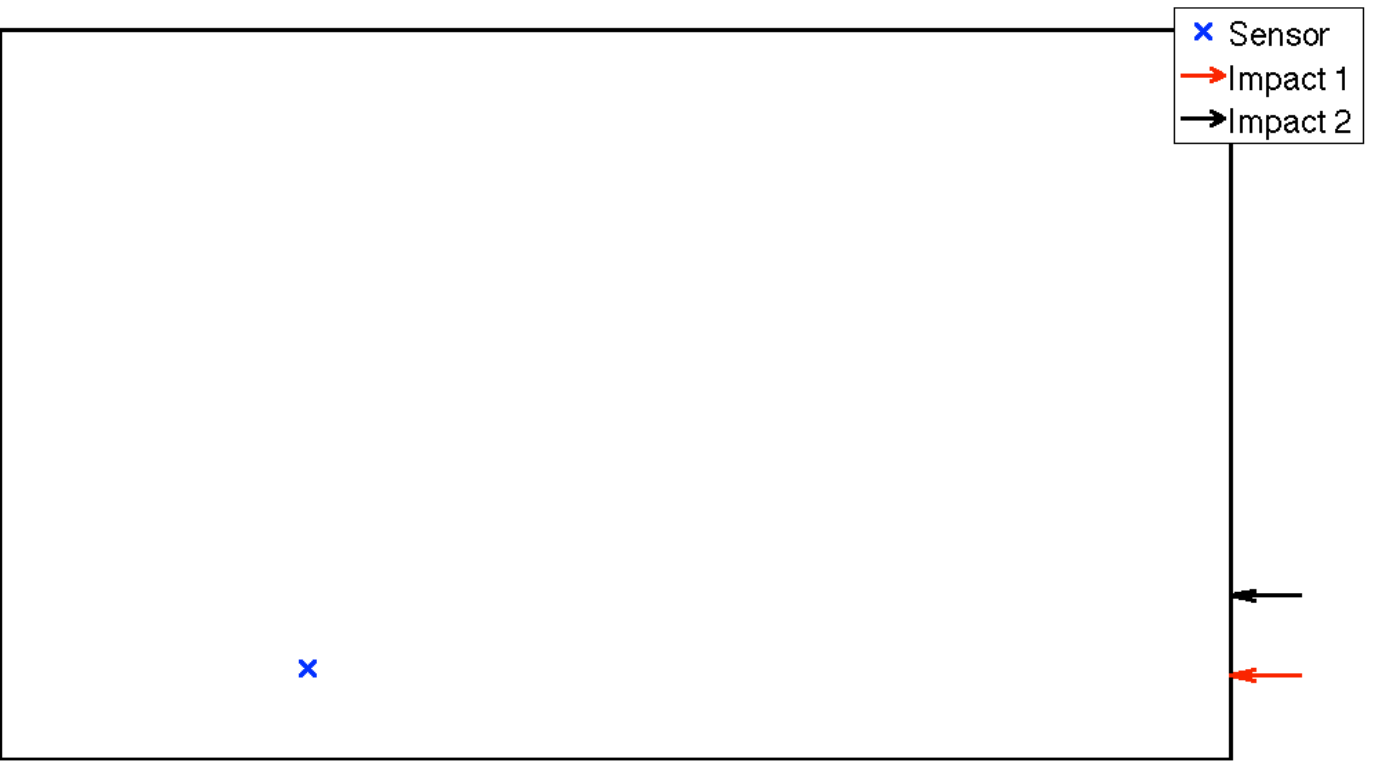

Figure 3.18 Impact locations for in plane impact location test.

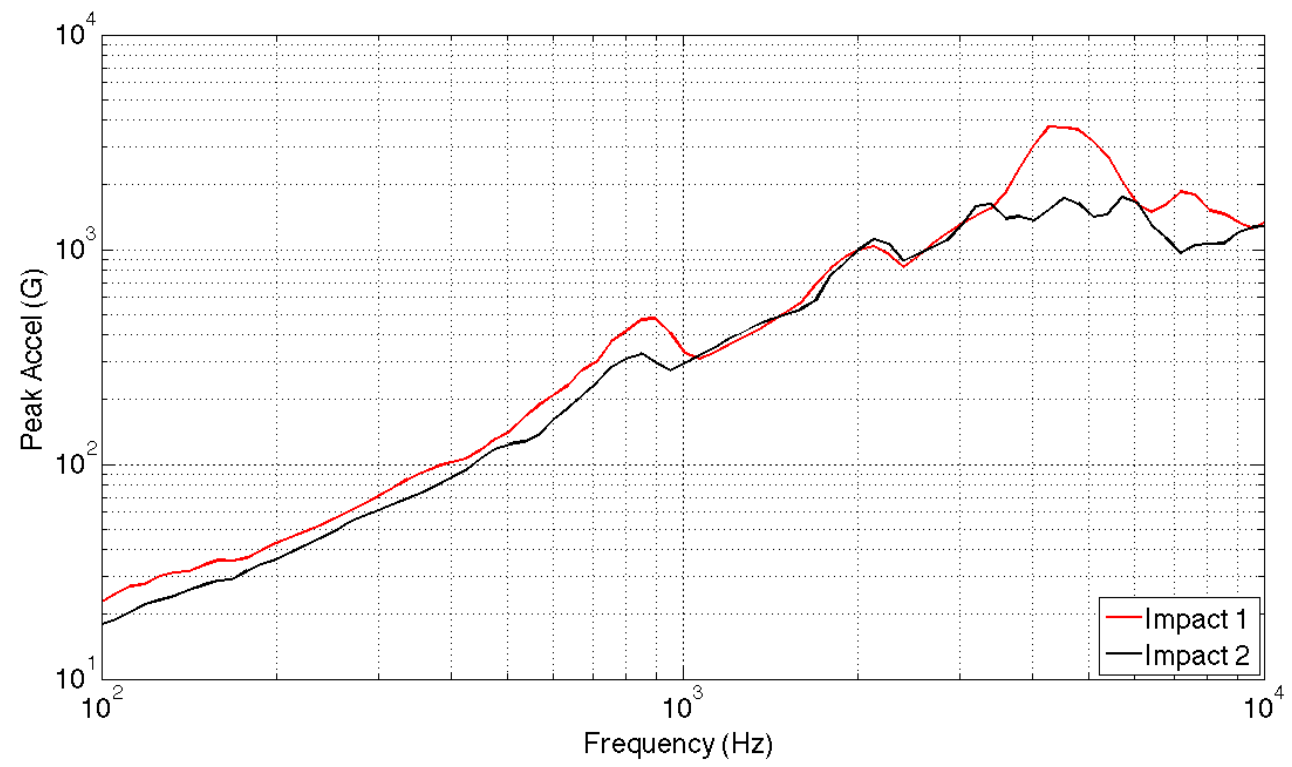

Figure 3.19 In plane impact location test.

The two curves have almost the exact same shape from 100 to $2500 \mathrm{~Hz}$. From 100 to $1000 \mathrm{~Hz}$ impact location 1 had a slightly higher magnitude, but this follows from the observations made in section 3.7.2 about impacts farther from the middle of the plate doing a better job of exciting the mode that moves diagonally across the plate. Even 
though the magnitudes are slightly different, the shapes match almost perfectly. Nothing interesting happens until beyond $3000 \mathrm{~Hz}$, where the two impacts clearly excited different high frequency modes. The author conducted more tests that are not presented here across different sensor locations and impact locations. All of the results were the same: similar shapes until around $3000 \mathrm{~Hz}$, beyond which the curve shapes could vary greatly. This parameter could help move the high frequency portion of a test SRS within tolerance of a requirement SRS, and it can yield larger magnitude changes than the other parameters discussed to this point.

\subsection{Interface Plate}

These last three sections are less parameters that can be varied and more preparing for an actual test with a real test piece. It is very unlikely that a test piece would have the same mounting pattern as the shock table apparatus. Therefore an interface plate must be used that can attach to the apparatus, provide a mounting point for the test piece, and allow the accelerometers to be mounted. Ideally the accelerometers would mount as close as possible to the test piece in order to give the best possible measurement of the accelerations actually acting on the test piece. It is important that the test piece be rigidly attached to the interface as this will ensure that the accelerations are transferred from the interface to the test piece as well as possible. Figure 3.20 presents the interface used for this section and section 3.10. Note that for this facility's use, ordinarily the interface would be just a plate. The test piece would be inside essentially a box, and the box would be what attaches to the interface. However, the author needed a way to add mass to the interface to simulate a test piece, so the box was built in to the plate. 


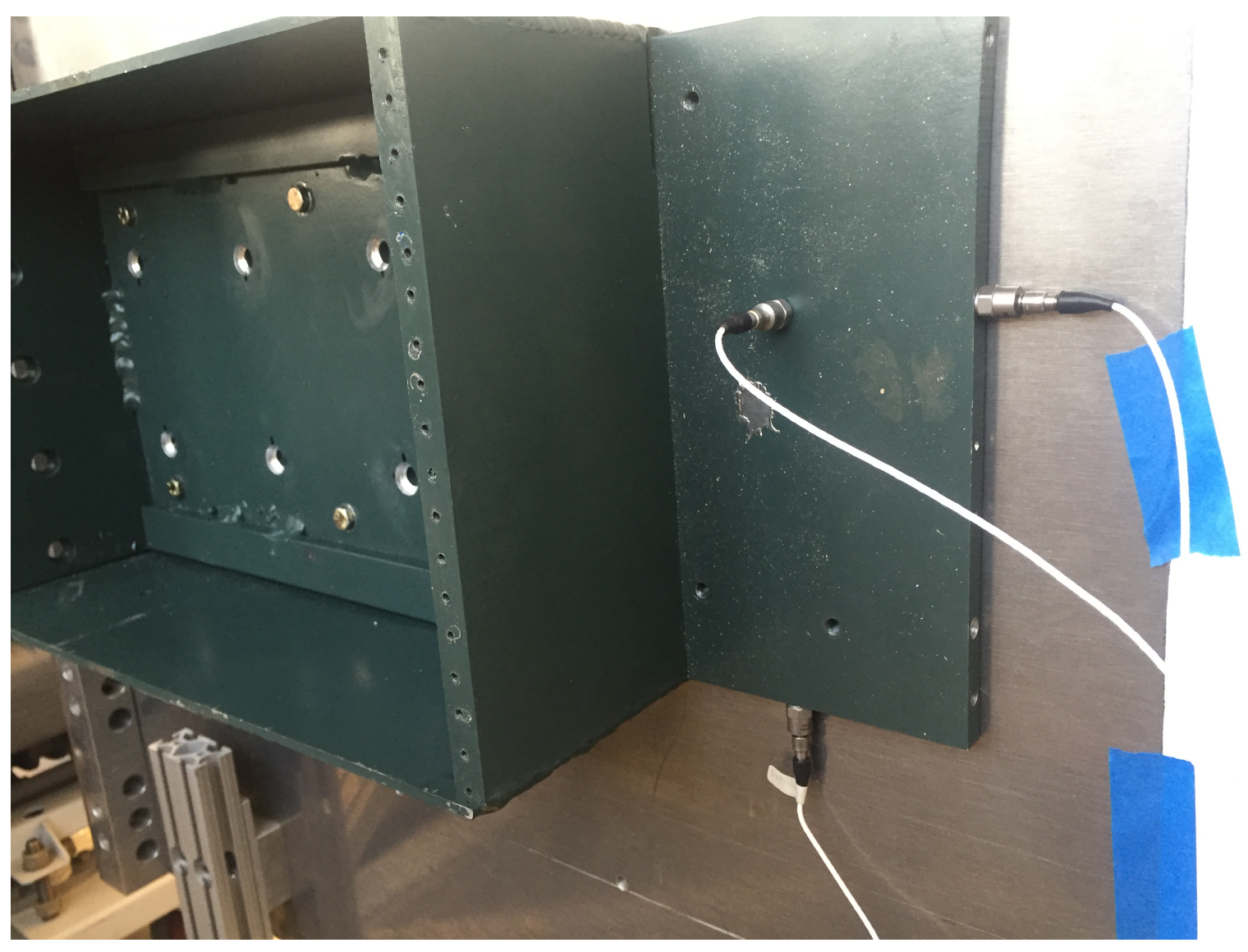

Figure 3.20 Example interface plate.

\subsubsection{Out of Plane}

The interface plate was expected to drastically change the resulting SRS. No matter how small or simple the interface is, it will still have its own modes and it will attenuate the acceleration compared to the aluminum plate. Note that section 3.9 compares the results of an impact where the accelerometers were mounted to the aluminum plate with an impact where the accelerometers were mounted to the interface plate. Since it is impossible to mount the accelerometers in the same place for both cases, the interface plate was roughly centered over the location on the aluminum plate where the accelerometers were mounted. 
This test was run with the first set of repeatability plates and a release angle of 34.4 degrees. One curve was generated without an interface plate and the other was generated with an interface plate, as shown in Figure 3.21.

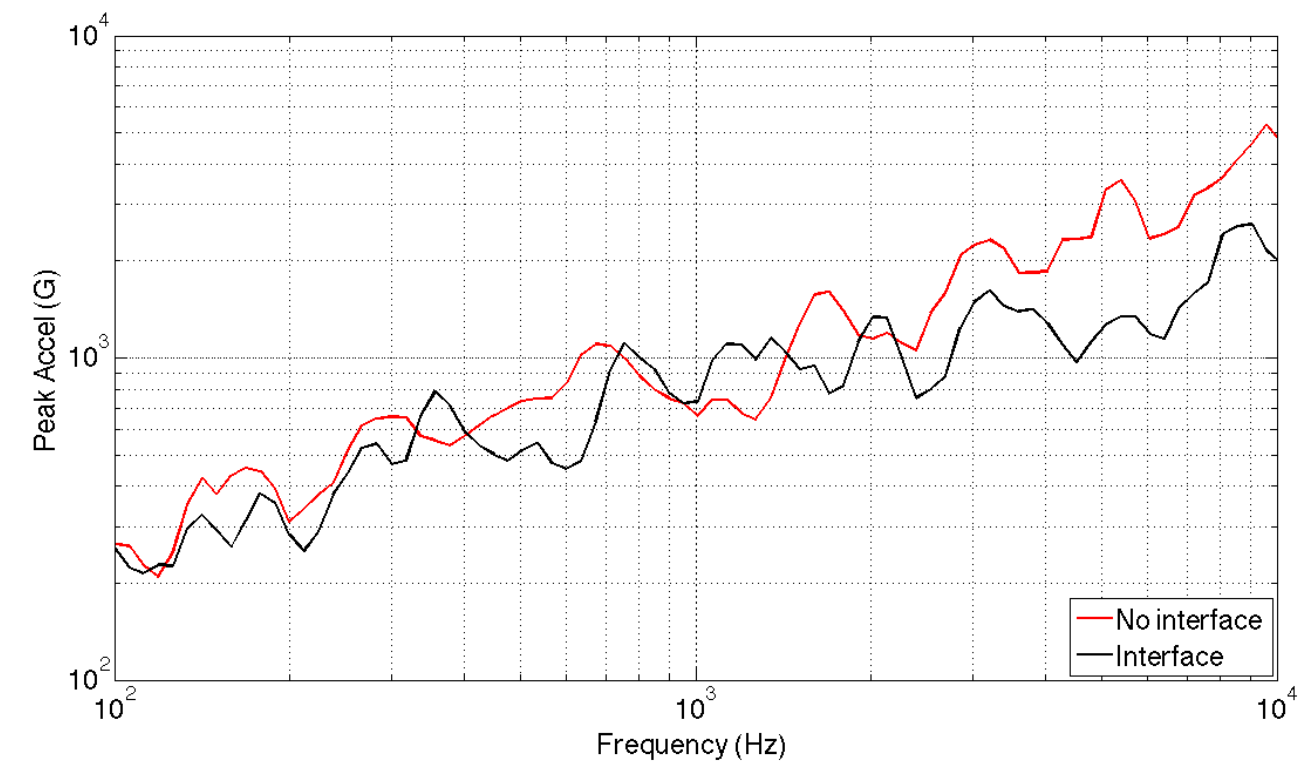

Figure 3.21 Out of plane interface plate test.

Surprisingly the interface plate did not reduce the SRS magnitude as much as anticipated. The curves are very similar magnitude up to about $2500 \mathrm{~Hz}$, beyond which they finally begin to exhibit a noticeable amplitude difference. The interface did introduce new modes as expected; these can be seen in the form of curve peaks at different frequencies. The general shape of the curves is very similar though, which was not expected. This was an encouraging test, as it gave hope that the large number of tests done without an interface would provide good predictive powers to an actual test.

\subsubsection{In Plane}

Since the interface should attenuate the accelerations that reach the accelerometer, the SRS should drop in magnitude. Additionally, the curve shape should change due to the addition of new modes. 
A release angle of 46.0 degrees as measured by the first set of repeatability plates resulted in the curves presented in Figure 3.22. The curves represent measurements taken with and without an interface plate.

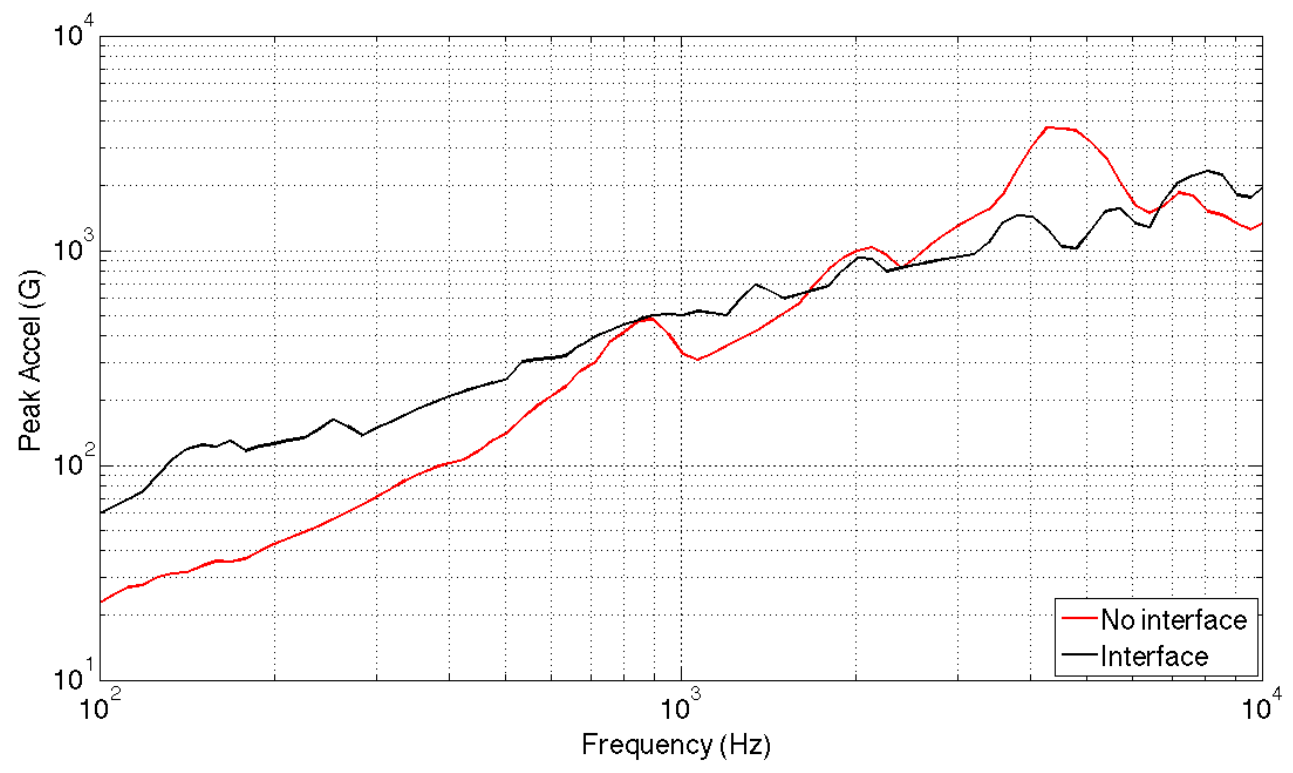

Figure 3.22 In plane interface plate test.

This test resulted in large changes to the SRS as expected, though the form of the changes was not expected. From 100 to about $1500 \mathrm{~Hz}$, the interface actually resulted in a larger magnitude curve with a less pronounced slope. This could mean that the in plane impact excited a powerful structural mode in the interface plate that resulted in more low frequency vibration than is typical of the aluminum plate. The interface did introduce more high frequency modes, as evidenced by several peaks in the curve above $3000 \mathrm{~Hz}$. The overall curve shape with the interface plate is very similar to the curves that were characteristic of the out of plane impacts discussed in previous parameter sections. While this is unlikely to translate to the trends observed in those out of plane parameters applying to this in plane interface test, it is interesting to note. 


\subsection{Test Piece Mass}

The following tests were simulations of an actual test, complete with the interface plate shown in Figure 3.20 and a structure built as part of another student's thesis. This structure was meant to imitate a $6 \mathrm{U}$ CubeSat and fit into the interface shown in Figure 3.20. It added additional mass to the interface, just as a test piece would to its interface during an actual test. This test's goal was not to meet a requirement SRS with a set of tolerances; that will be discussed in section 3.11. The goal here was simply to observe whether or not adding mass to an interface would significantly affect the SRS. This would be useful information to have as it would allow the user to attempt to compensate for this effect while running tests with just the interface plate. This could help reduce the amount of trial and error that goes into generating an acceptable SRS and reduce the number of equalization tests performed on the test dummy prior to an actual test with a real test piece. Too many equalization tests could break a test dummy, so limiting the number of shock events it experiences would be beneficial.

\subsubsection{Out of Plane}

The addition of mass to the interface plate was hypothesized to have little to no effect on the shape of the SRS. Instead, it should simply reduce the curve's magnitude uniformly across the entire frequency range. This would be a useful result as it would make compensating for additional mass on the interface plate as simple as changing the release angle.

The first set of repeatability plates were used to verify a release angle of 34.4 degrees. Figure 3.23 depicts the results of adding mass in the form of a test piece to an interface plate. 


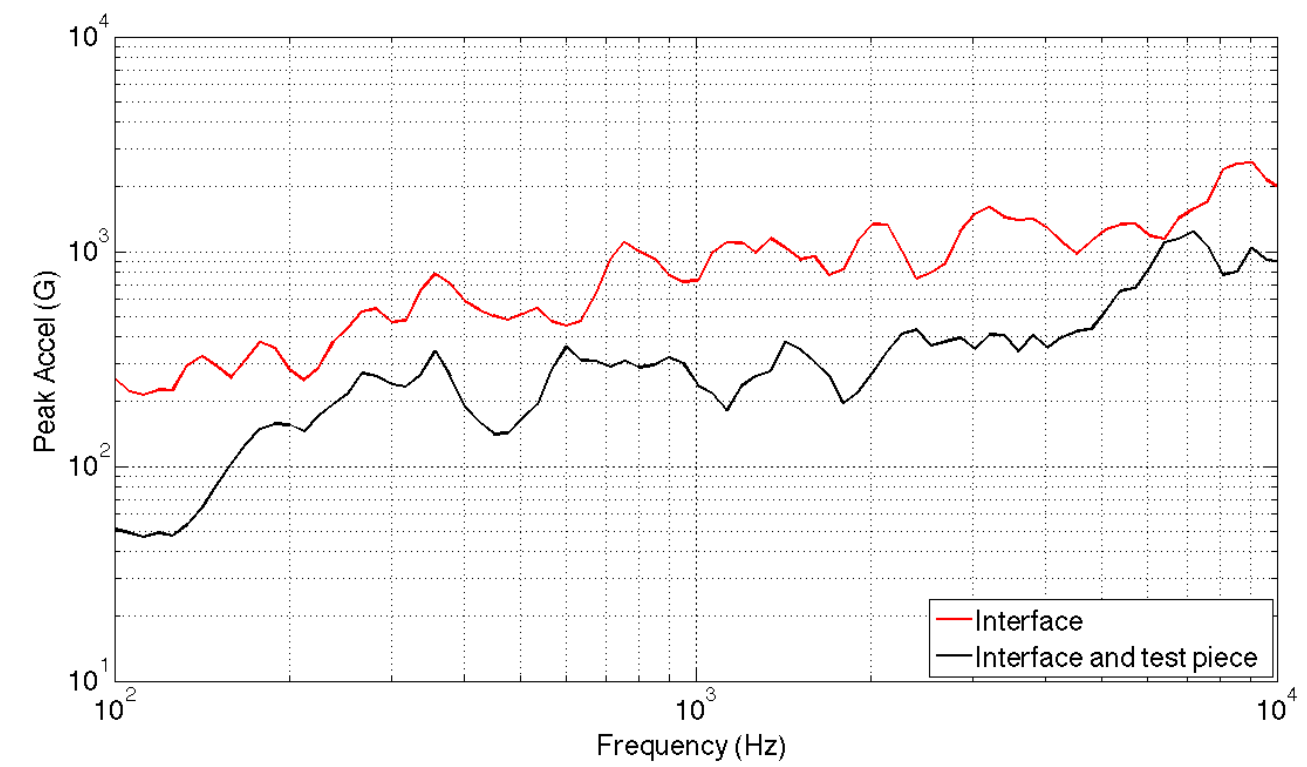

Figure 3.23 Out of plane test piece mass test.

The results supported the hypothesis. The two curves exhibit similar overall shapes across the frequency range. The peaks are not perfectly aligned, but most of the peaks in one curve seem to have a counterpart in the other. The curve representing a test piece being attached to the interface plate did exhibit a decrease in magnitude, and it was nearly the same drop across all frequencies. The decrease was a little higher from 100 to $170 \mathrm{~Hz}$ and a little lower from 6000 to $10000 \mathrm{~Hz}$ than across the rest of the frequencies. Overall this test was encouraging, as it followed the hypothesis well. The hypothesis would allow for relatively easy modification between tests with and without a test piece mounted to an interface.

\subsubsection{In Plane}

The relative behavior was hoped to be the same for an in plane impact as it was for the out of plane test. The shape should remain roughly the same, but the magnitude should decrease with more mass attached to the interface. This decrease was expected to be smaller for the in plane test than it was for the out of plane test. 
This test was conducted with the first set of repeatability plates and a release angle of 55.0 degrees. Two impacts were performed; one with just the interface plate and one with the test piece attached to the interface plate. The resultant curves are shown in Figure 3.24.

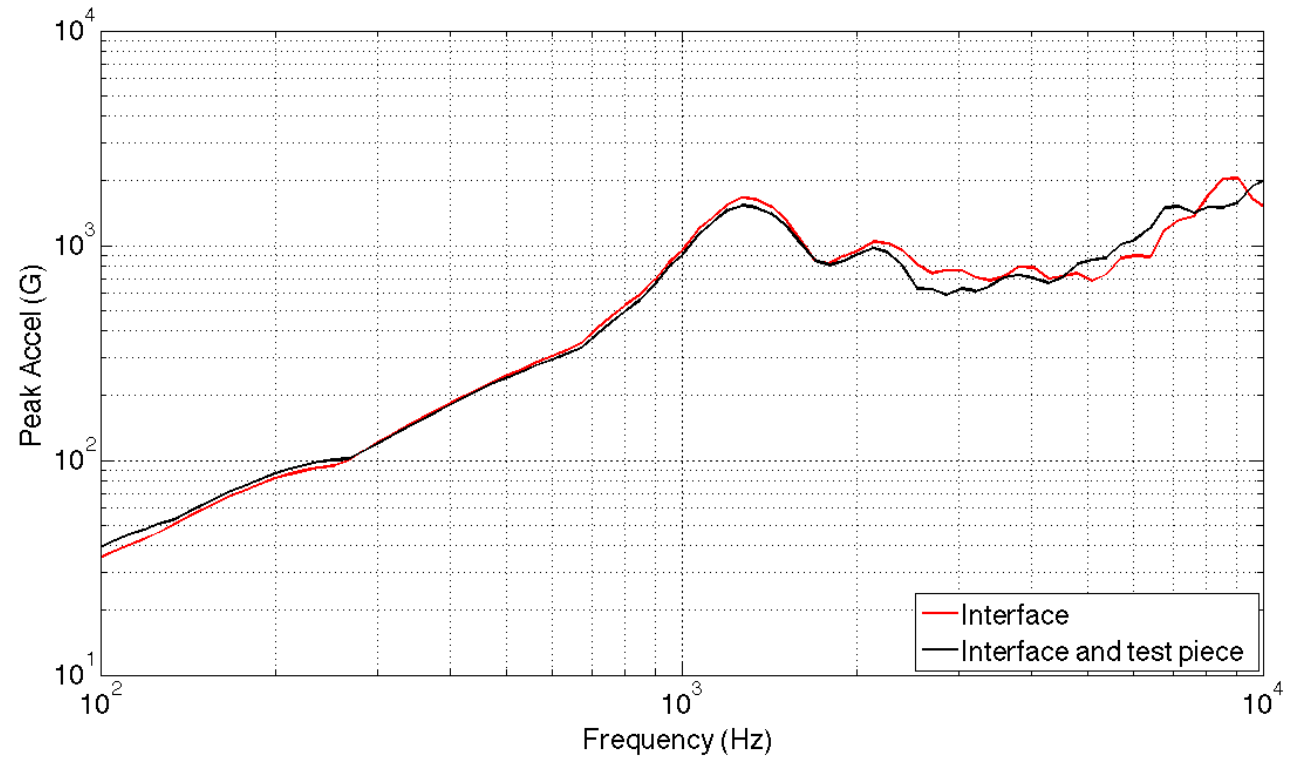

Figure 3.24 In plane test piece mass test.

These results were not expected, but even better than the author was hoping for. From 100 to about $2000 \mathrm{~Hz}$ the curves not only follow the same shape but have almost the exact same magnitude. From 2000 to $10000 \mathrm{~Hz}$ the curves are not as similar as in the lower frequencies, but still differ at the most by less than $3 \mathrm{~dB}$. The average difference across that frequency range is even smaller: about $1 \mathrm{~dB}$. The curves do show signs of different modes being excited in those upper frequencies, but the magnitude difference between them is so small it can almost be ignored. This test yielded excellent results as it supports a possible decision to perform the equalization tests with no test piece attached. The user could then do one test with a dummy mass to ensure that the results were as expected, then move to the actual test piece. It should be noted that this test only used one 
interface. It is possible that other interfaces would behave differently; further testing is needed before these results can be generalized to all interfaces attached to this shock test apparatus.

\subsection{CubeSat Tests}

This section describes the culmination of the project. The author attempted to meet a given SRS requirement with $+6 /-3 \mathrm{~dB}$ tolerance bounds for a $3 \mathrm{U}$ CubeSat. The equalization tests were done on a Test-POD with a mass simulator inside to represent the CubeSat. Figure 3.25 shows the Test-POD mounted to an interface plate, which was mounted to the shock table apparatus's aluminum plate. The accelerometers were mounted to the interface plate with a mounting block.

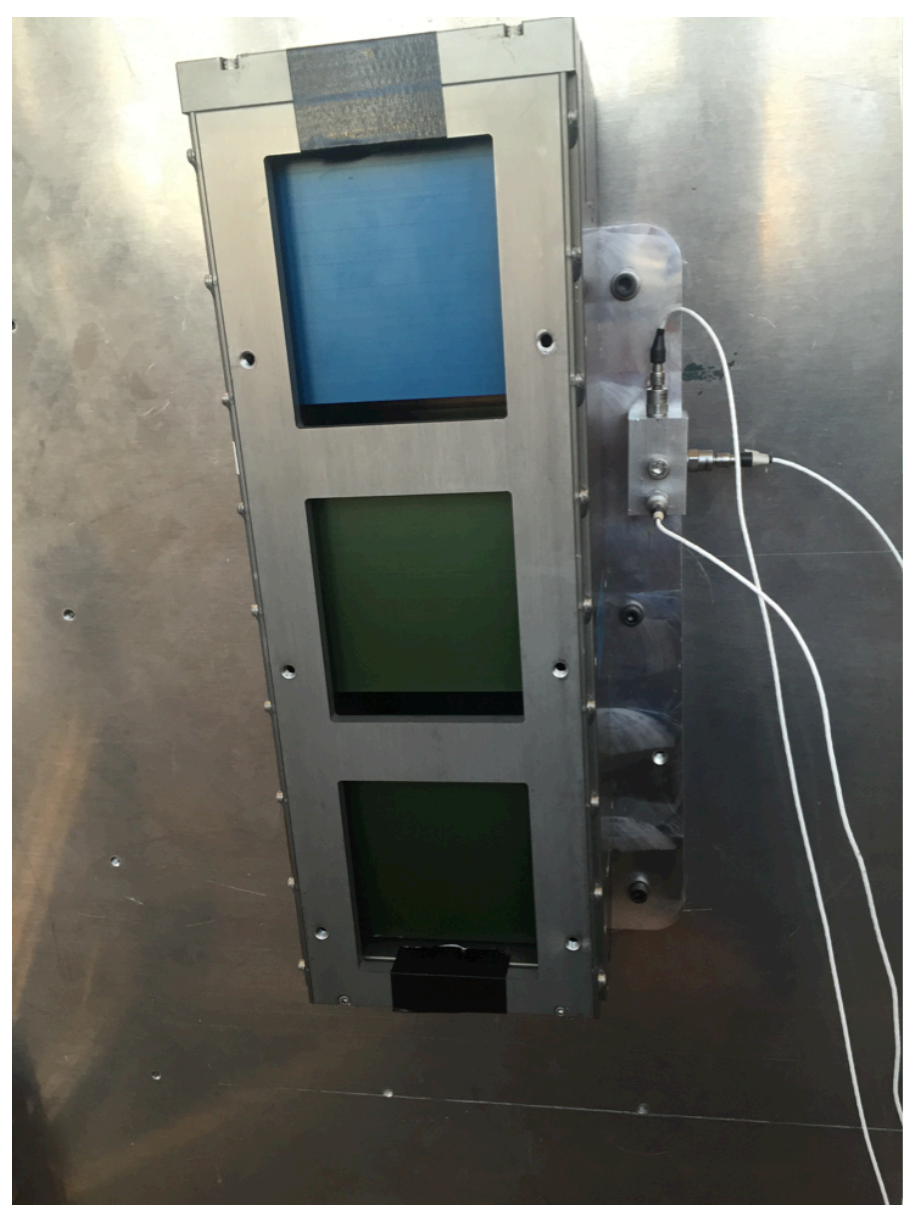

Figure 3.25 Test-POD mounted to shock table. 
Unfortunately these tests were attempting to meet a proprietary SRS requirement, which cannot be presented in this thesis. An example of the author's alternative to the usual SRS plot is presented in Figure 3.26. It shows the difference in dB between the target SRS requirement and both the positive and negative SRS curves for each frequency. If the test data was larger in magnitude than the test requirement, then the $\mathrm{dB}$ difference was positive, with the opposite being true for a negative $\mathrm{dB}$ value. The test requirement had a lower tolerance bound of $3 \mathrm{~dB}$ and an upper tolerance bound of $6 \mathrm{~dB}$. At least $50 \%$ of the test frequencies were required to have magnitudes above the requirement SRS, which in these plots is indicated by a positive $\mathrm{dB}$ value.

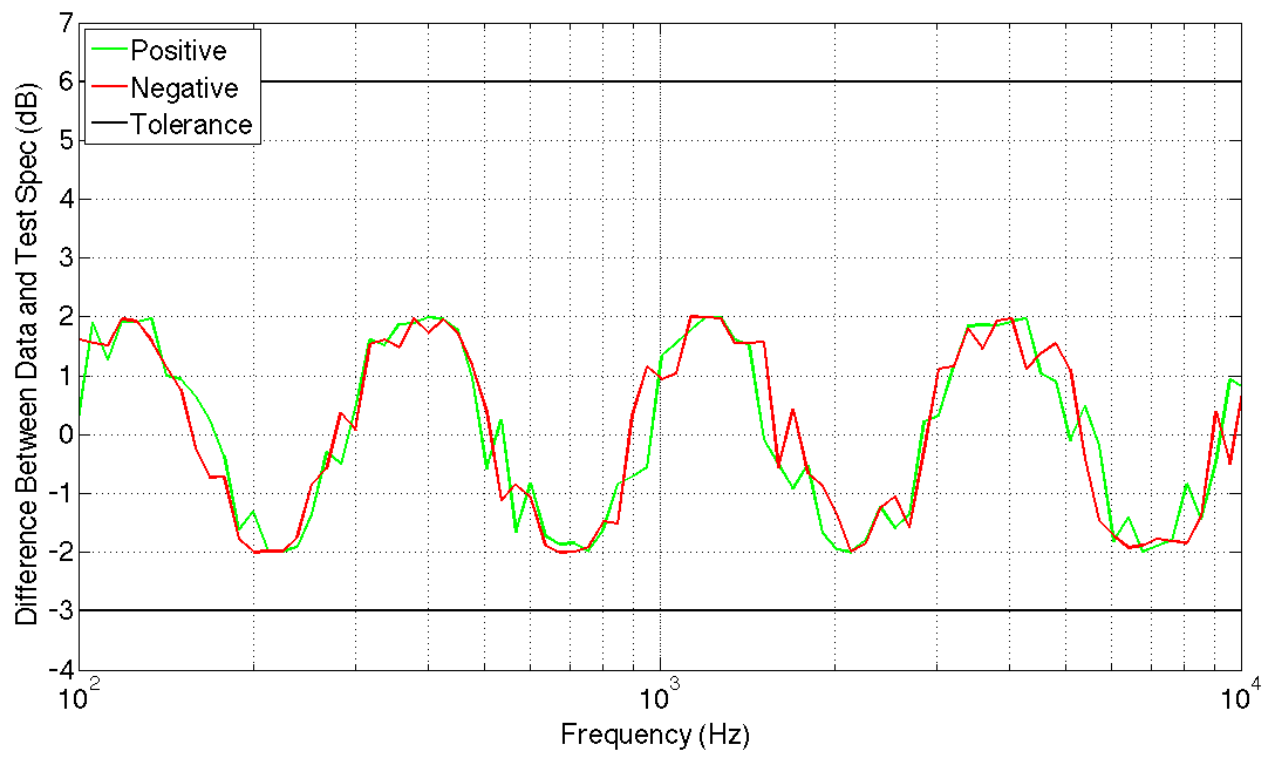

Figure 3.26 SRS requirement with tolerances.

The two following sections will detail the attempts to meet these requirements. Note that successfully meeting the requirements would be indicated in this type of plot by the entirety of both curves being between the two black tolerance bounds and by more of the curves being positive than negative. Dozens of tests were run in both the out of plane and in plane configurations while working to meet the requirement SRS. For each 
configuration four tests are presented in an effort to portray the testing flow and the change and progress from test to test.

\subsubsection{Out of Plane}

The first attempt with the Test-POD mounted is shown in Figure 3.27. It was run with a release angle of 37.4 degrees and did not have a mechanical filter at the impact location or any extra mass mounted to the hammer. Even with a relatively low release angle, the positive and negative curves do not match perfectly in the lower frequencies, indicating a zero shift in the data. This would become a theme of the testing, as zero shift continually showed up in the data. The author believes that these issues may have been caused by either base strain in the accelerometer housing due to an imperfect mounting surface or overloading the accelerometer due to some high energy, high frequency mode introduced by the combination of interface plate and Test-POD.

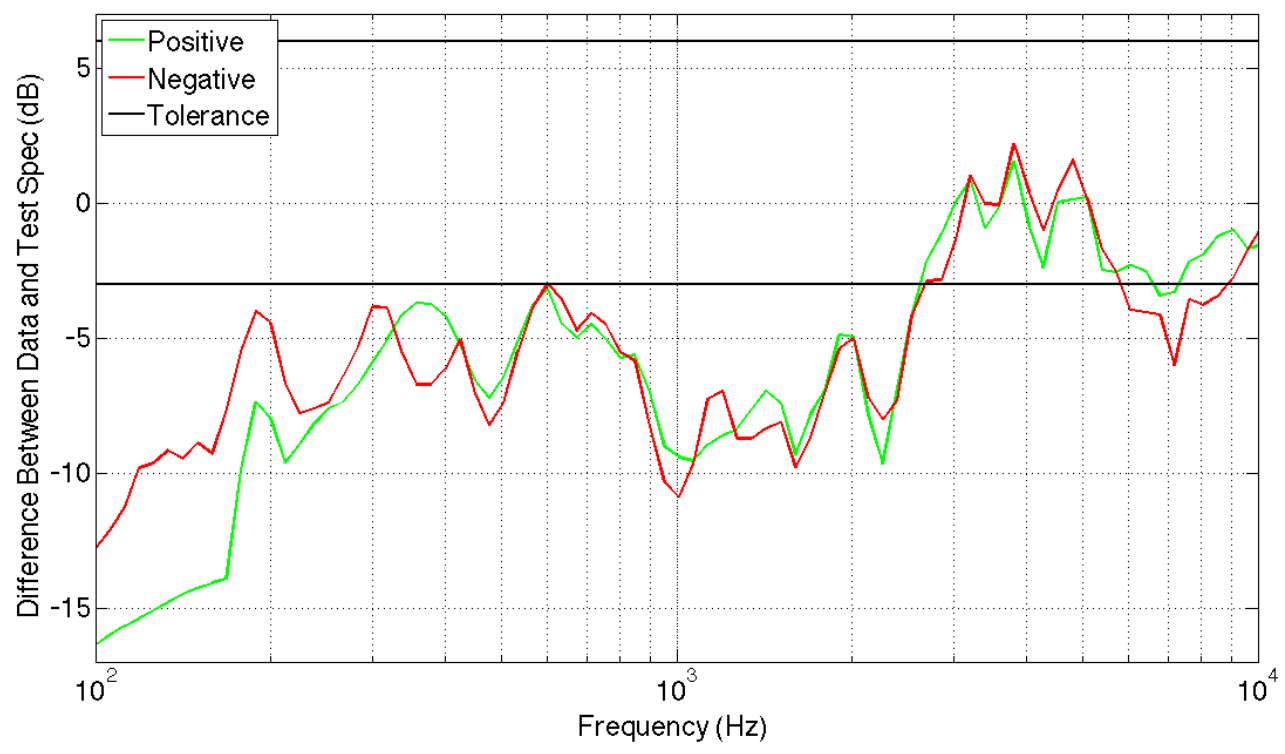

Figure 3.27 First out of plane attempt with the Test-POD.

The twenty-fifth attempt is shown in Figure 3.28. The pendulum arm was released from 46.3 degrees with $15 \mathrm{~kg}$ of extra mass and no mechanical filter. The SRS curves 
were closer in magnitude to the requirement as indicated by the smaller $\mathrm{dB}$ values, but there is still evidence of a zero shift in the data as evidenced by the lower frequency disagreement between the positive and negative curves. Also note that from about 2000 to $10000 \mathrm{~Hz}$ the curves are closer to the upper tolerance than they are to the lower tolerance in the lower frequencies. This could lead to over-testing in the upper frequencies if the release angle were increased, which would require a change to another parameter to be made.

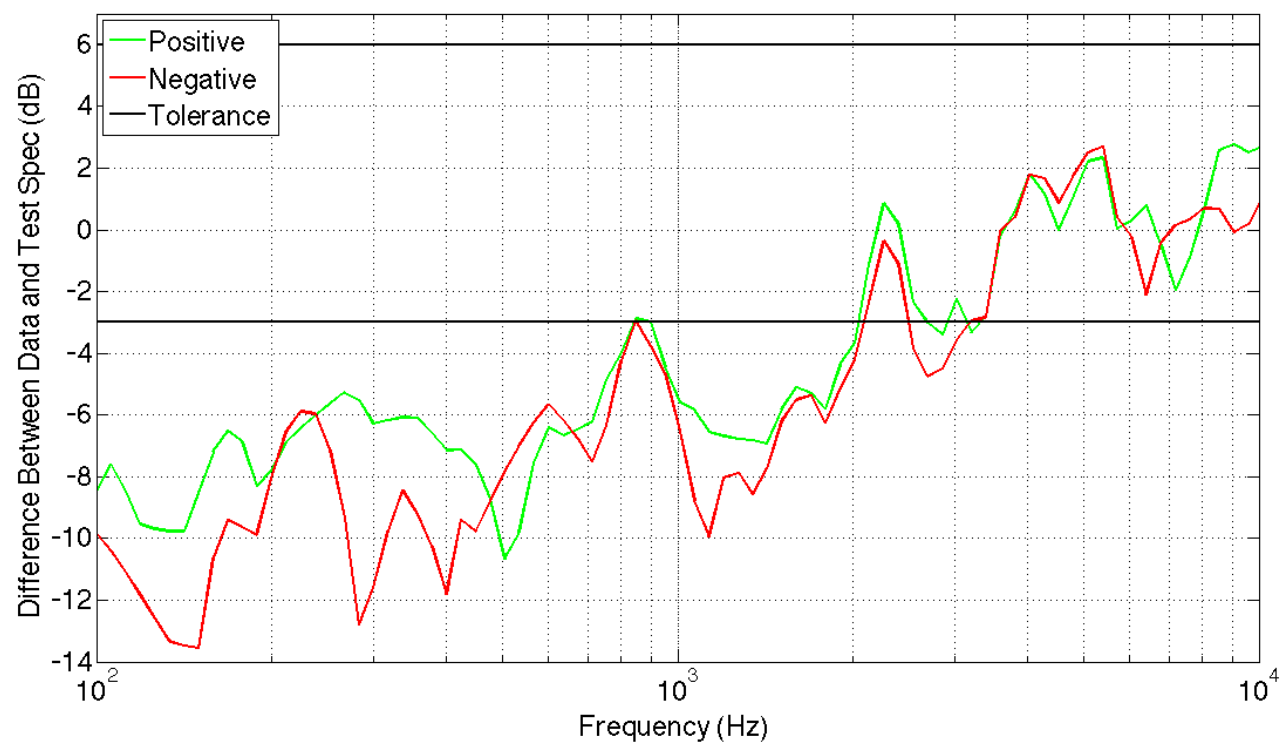

Figure 3.28 Twenty-fifth out of plane attempt with the Test-POD.

Figure 3.29 shows the fifty-seventh attempt. It used an 83.5 degree release angle with $10.6 \mathrm{~kg}$ of extra mass added to the hammer and 1/4-inch of cardboard at the impact location.

At first glance this set of curves seems very close to meeting the requirement, with only a few peaks and valleys being out of tolerance. However, there is still a slight indication of zero shift from about 300 to $900 \mathrm{~Hz}$. These parameters were close, but needed a little more modification to result in an acceptable SRS. 


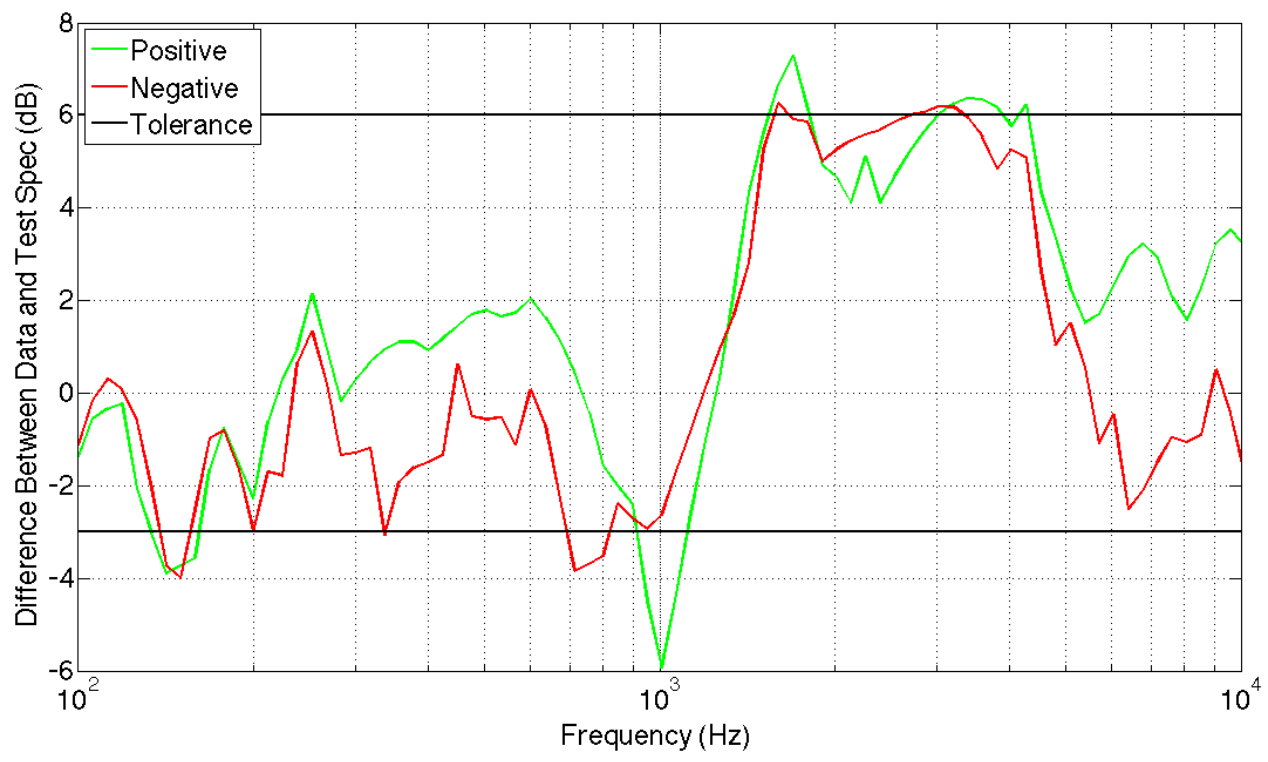

Figure 3.29 Fifty-seventh out of plane attempt with the Test-POD.

Attempt number sixty-five is shown in Figure 3.30. Like the previous discussed attempt, it had a release angle of 83.5 degrees with $10.6 \mathrm{~kg}$ of extra mass and 1/4-inch of cardboard at the impact location. It should be noted that the impact location and location of the Test-POD were continually being modified throughout these tests.

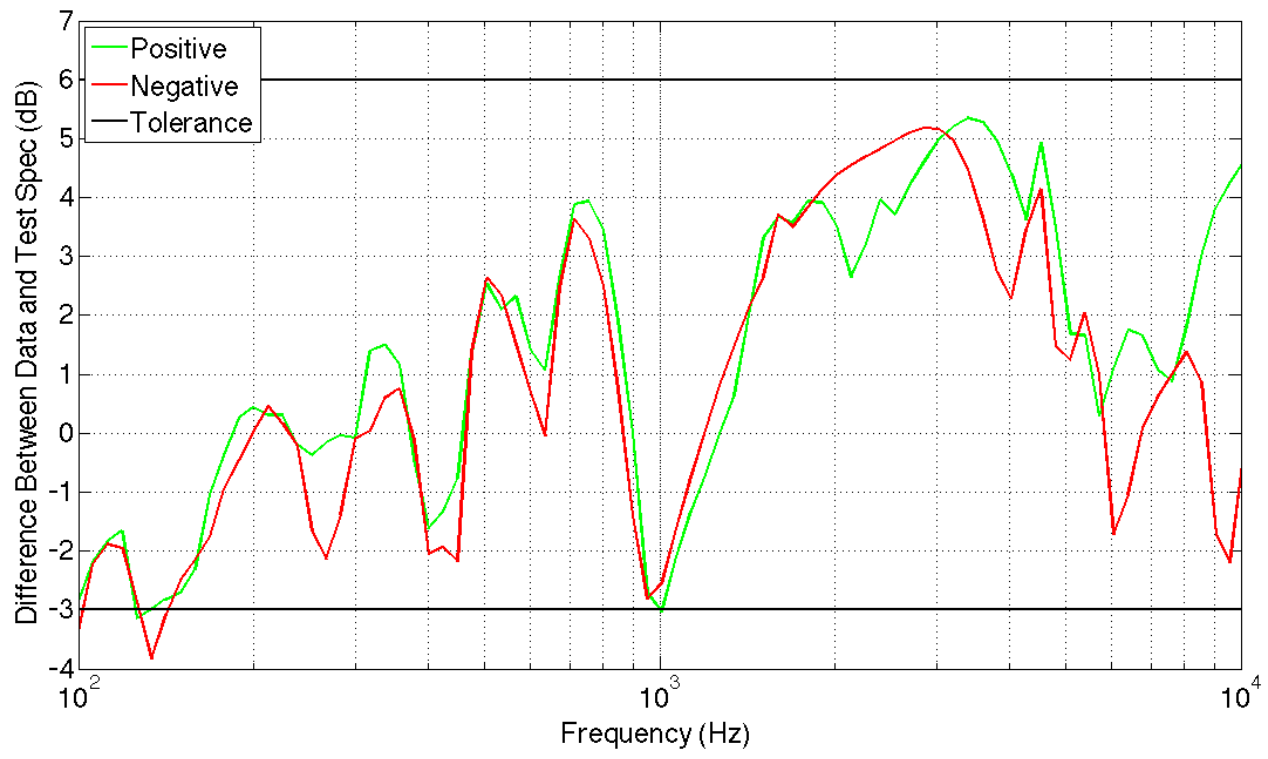

Figure 3.30 Sixty-fifth out of plane attempt with the Test-POD. 
The zero shift was finally eliminated and other than a small under-test from 120 to $160 \mathrm{~Hz}$, both curves are entirely inside the tolerances. That small disagreement could be fixed with a little more hammer mass or a slight increase in the release angle. Additionally, $71.6 \%$ of the frequencies had magnitudes greater than the requirement SRS as evidenced by the abundance of positive $\mathrm{dB}$ values. This test was considered a success.

\subsubsection{In Plane}

The first attempt to meet the test requirement with an in plane strike used a release angle of 52.0 degrees with no extra hammer mass and no mechanical filter at the impact location. Figure 3.31 shows the results, and it portends issues to come. There is an obvious zero shift as indicated by the large difference between the positive and negative curves from 100 to $900 \mathrm{~Hz}$. Also, the high frequency portions of the curves are very close to the requirement while the lower frequency portions are as much as $22 \mathrm{~dB}$ from the requirement. Even if the zero shift were not present, it would likely be difficult to get the lower and upper frequencies in tolerance at the same time.

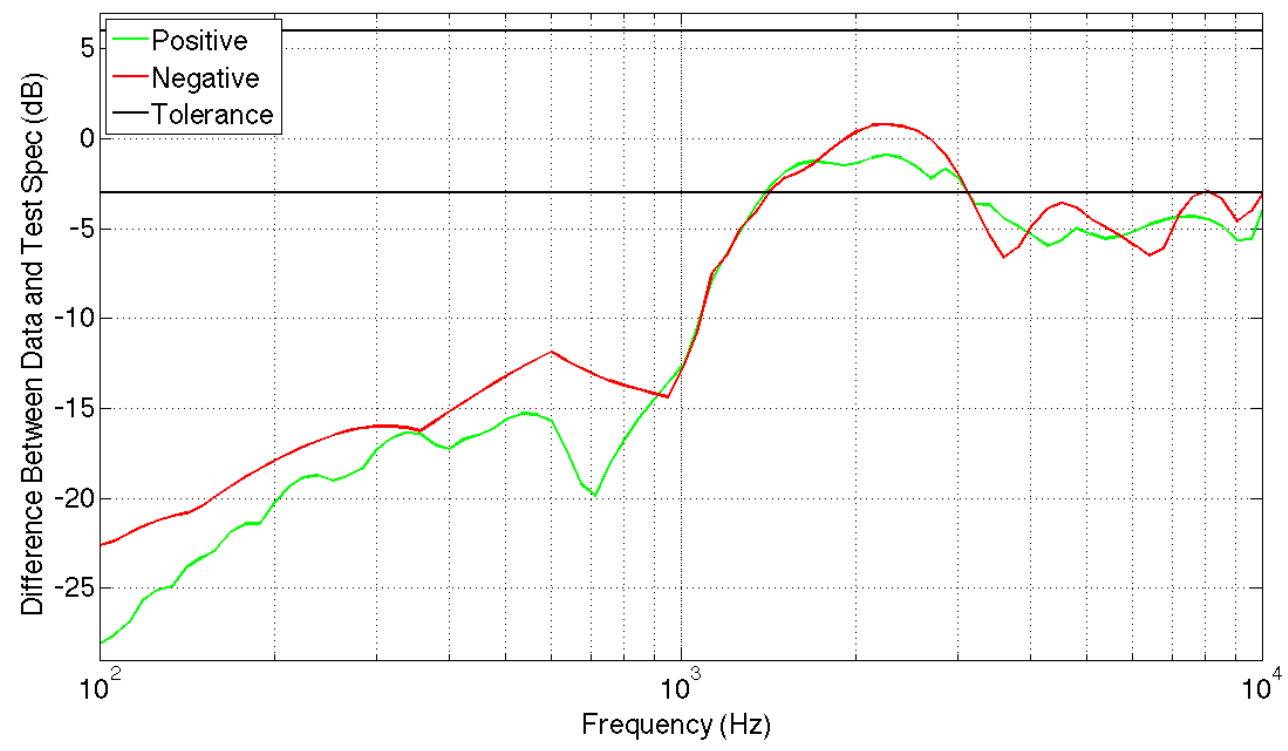

Figure 3.31 First in plane attempt with the Test-POD. 
The fifteenth attempt was released from 72.5 degrees with $10.6 \mathrm{~kg}$ of mass added to the hammer and no mechanical filter at the impact point. Figure 3.32 displays the extreme zero shift present in this attempt. At about $180 \mathrm{~Hz}$ there was nearly a $100 \mathrm{~dB}$ difference between the positive and negative curves, with another $50 \mathrm{~dB}$ difference around $350 \mathrm{~Hz}$. While zero shift was a minor issue in the out of plane testing as well, this zero shift's magnitude led the author to believe that the in plane impacts must produce some significant high frequency content that the accelerometers cannot handle.

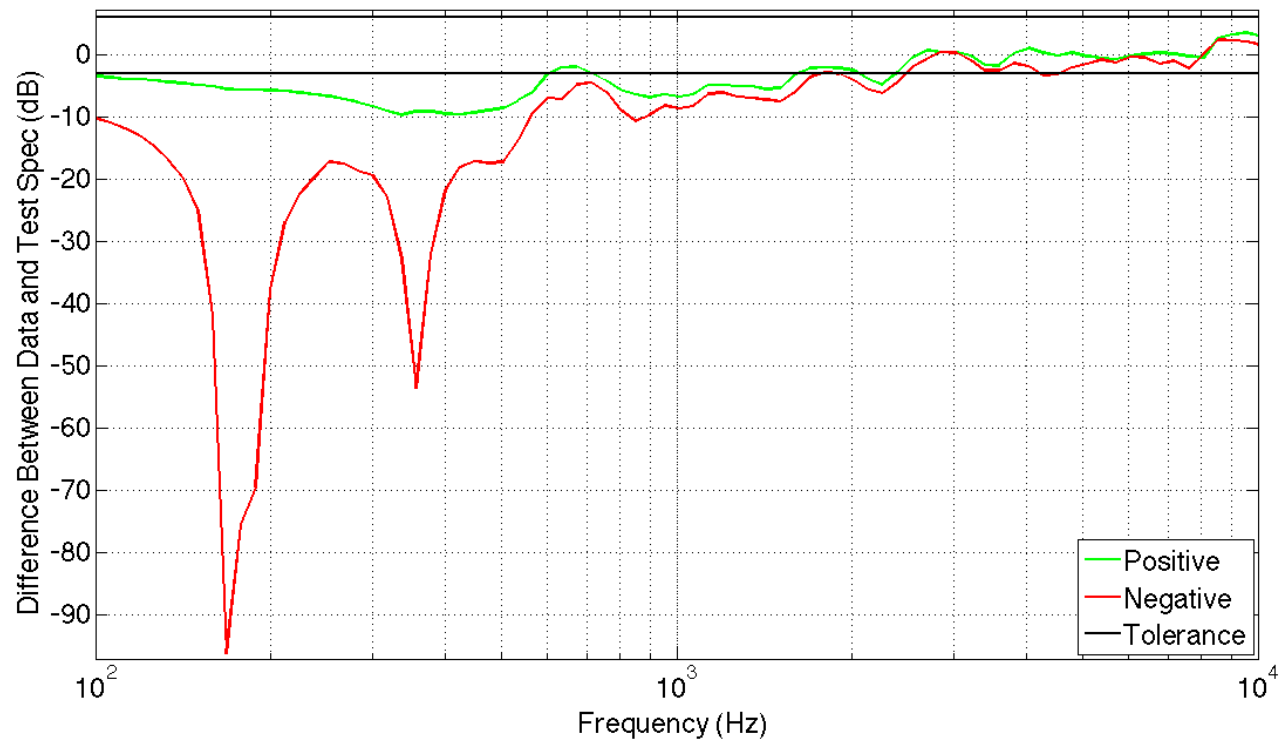

Figure 3.32 Fifteenth in plane attempt with the Test-POD.

Figure 3.33 displays the results of the nineteenth attempt. It used a release angle of 52.0 degrees with $10.6 \mathrm{~kg}$ of extra mass and 1/4-inch of cardboard at the impact point. There is still a zero shift in the Figure 3.33 data, with the largest differences occurring at $600 \mathrm{~Hz}$ with a peak in the negative curve and at $700 \mathrm{~Hz}$ with a valley in the positive curve. Note that the curve shape still does not match the requirement SRS shape, with the high frequencies being much closer than the low frequencies. A perfectly shaped test would yield a horizontal line on these plots. 


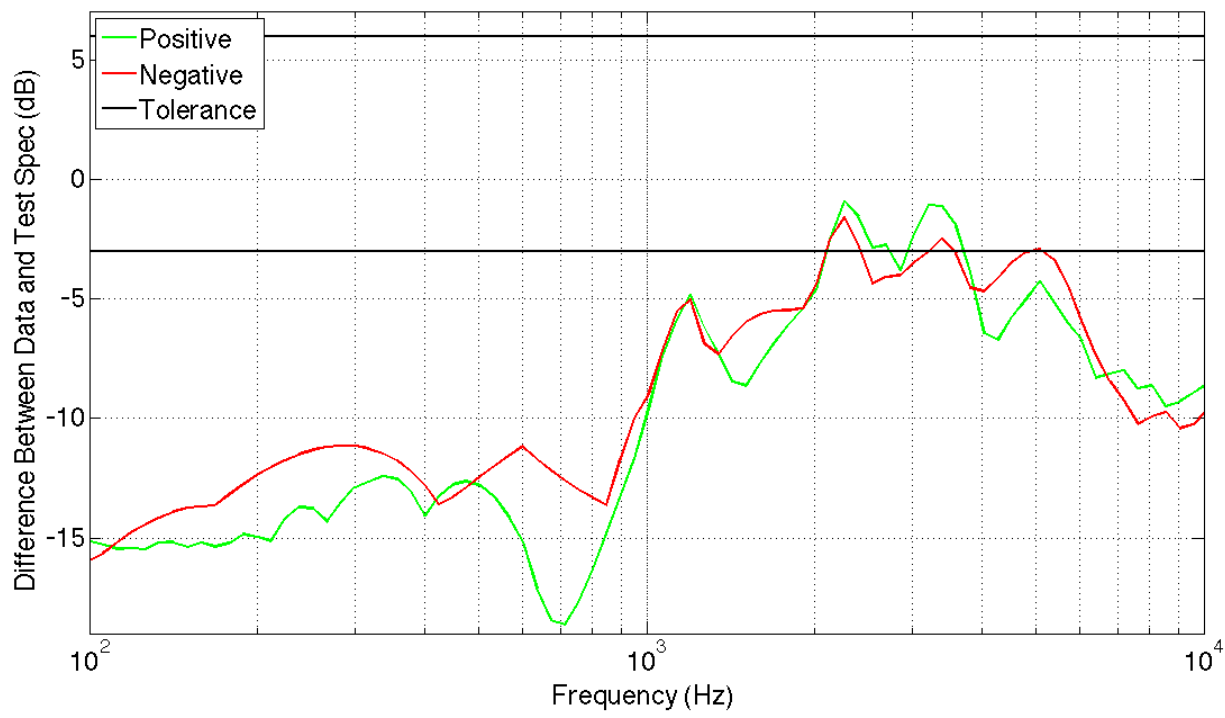

Figure 3.33 Nineteenth in plane attempt with the Test-POD.

The twenty-third attempt, presented in Figure 3.34, also saw the hammer dropped from 52.0 degrees with $10.6 \mathrm{~kg}$ of extra mass and 1/4-inch of cardboard as a filter. None of the many attempted combinations of impact location and test piece location alleviated the zero shift that was seen in the data. This was the best attempt and it did not have any frequencies inside the tolerance band. The in plane testing was considered a failure.

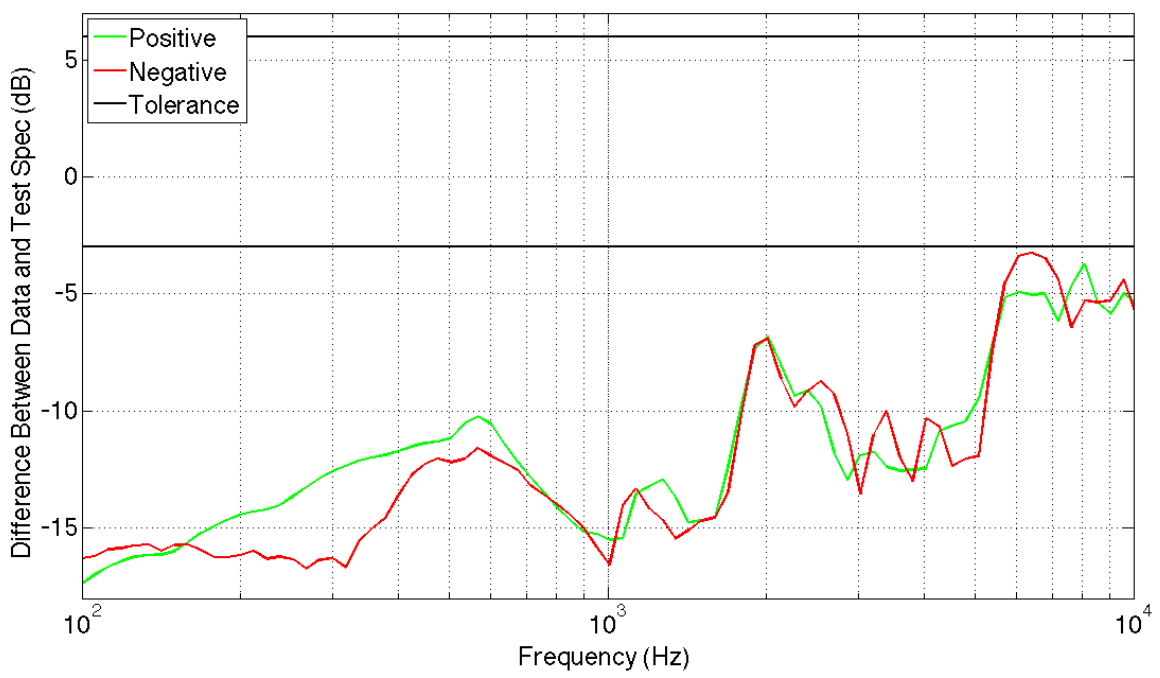

Figure 3.34 Twenty-third in plane attempt with the Test-POD. 


\section{FINITE ELEMENT MODEL AND ANALYSIS}

The author initially hoped to develop a finite element model (FEM) that could be used in conjunction with the database of prior tests to help eliminate trial and error for the end user. After discovering that dozens of tests could be performed in the time it took one analysis of the FEM to run, that hope was essentially abandoned and this chapter is mostly for academic purposes. Also, modeling the system could be useful if a major change is to be made to the apparatus, so it is with that in mind that this section is presented.

The models were created in SolidWorks 2015, and the finite element analysis (FEA) was conducted with the Simulation add-in for SolidWorks. The author realizes that higher accuracy results could likely be obtained from programs dedicated to FEM/FEA such as Ansys, Abaqus, or Dytran. Additionally, these programs all have the ability to model impacts, which would improve the results. However, the student editions of those commercial products all impose node restrictions that the author believes would have prevented accurate simulations from being run. The author's SolidWorks model already surpasses the limits imposed by those other programs and it does not have the hammer included as would be necessary for the other programs' impact testing.

\subsection{Model Development}

The model was made as simple as possible in an attempt to reduce the hours of run time required for each analysis. The only features of the apparatus that were modeled were the aluminum plate, the accelerometer mounting block, and any interface plates that would be used to attach test pieces to the apparatus. Including the frame that supports the aluminum plate likely would have improved results marginally, but based on the results 
obtained from the simple model the author deemed the potential improvements not worth the increase in analysis run time. Figure 4.1 shows the front of the FEM while Figure 4.2 shows the back.

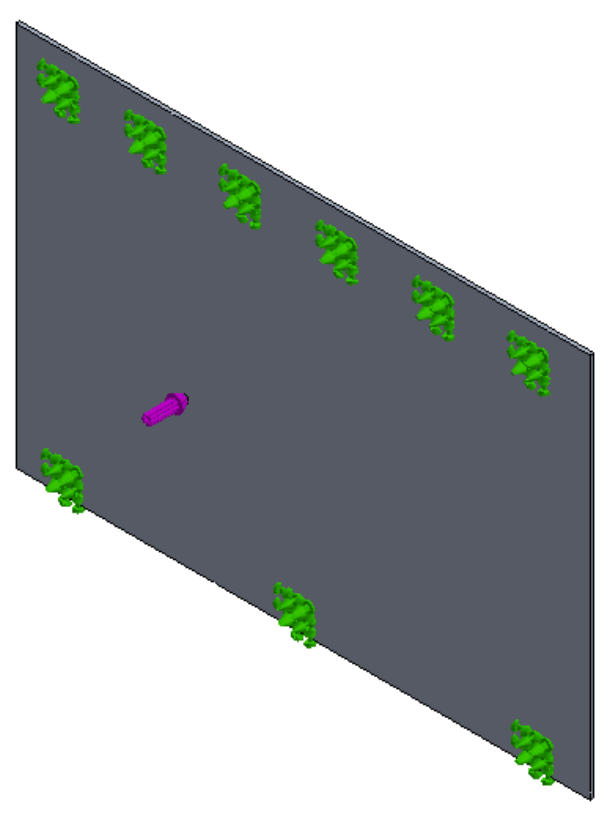

Figure 4.1 Front of finite element model.

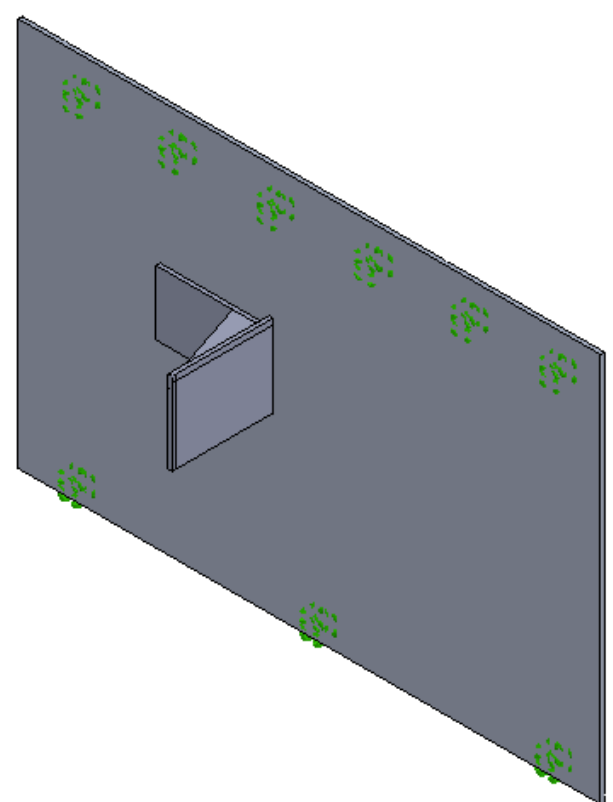

Figure 4.2 Back of finite element model.

The green squares are rigid fixtures and they represent the locations where the aluminum plate attaches to the frame. The purple arrow is the impact location, and impacts were modeled as a half sine pulse. The L-shaped assembly in Figure 4.2 would be the interface that a test piece gets mounted to. The interface plate used in the testing discussed in section 3.11 was flat, and the L-shaped interface shown is an idea to solve the apparatus's difficulties with in plane impacts and subsequent failure to meet the test requirement in all three axes. It will be discussed further in sections 4.2 and 5.2. The analysis was performed with Simulation's Dynamic Modal Time History. 
Since shock is a time-varying phenomenon, the static analysis that is synonymous with FEA would not suffice. Since time was now a factor in the analysis, the author first conducted a time step study to determine the maximum possible time step that still produced good results. Note that for a given analysis, the same step size was used for every time step, from the beginning to the end of the analysis. The study was done with Simulation's default mesh size, which resulted in 6731 nodes. Since the model's ultimate goal was to predict SRS curves for various inputs, acceleration data was collected at a single point for all analyses and that data was converted into an SRS. An adequate time step would be found when the SRS stopped exhibiting major changes between time step sizes. Table 4.1 displays the progression of time step sizes and the corresponding maximum $\mathrm{dB}$ change between subsequent runs.

Table 4.1 Time step study.

\begin{tabular}{|l|c|c|c|c|c|}
\hline Attempt & 1 & 2 & 3 & 4 & 5 \\
\hline Time Step (sec) & $3 \mathrm{e}-4$ & $1 \mathrm{e}-4$ & $5 \mathrm{e}-5$ & $3 \mathrm{e}-5$ & $1 \mathrm{e}-5$ \\
\hline$\Delta$ SRS (dB) & & 29.6 & 26.9 & 15.2 & 2.3 \\
\hline
\end{tabular}

Figure 4.3 presents the results of the time step study. All of the time steps resulted in reasonably similar curves up to about $900 \mathrm{~Hz}$. Beyond that it becomes apparent that the first two time step sizes were not large enough, as evidenced by the large oscillations in the upper frequencies that are not characteristic of pyroshock events. These time steps were too large because they essentially corresponded to sample rates of $3 \mathrm{kHz}$ and 10 $\mathrm{kHz}$, neither of which meets the bare minimum sample rate of $20 \mathrm{kHz}$. The last three time steps that were tried agreed very well until very high frequencies, above $4000 \mathrm{~Hz}$. There was still a large discrepancy between the third and fourth attempts at $10000 \mathrm{~Hz}$, but the 
fourth and fifth attempts agreed well across the entire frequency range. The fourth time step was chosen for several reasons. It corresponds to a sample rate of $33 \mathrm{kHz}$, which meets the minimum sample rate (note that aliasing was not a concern here since there was not actually any sampling occurring). The fifth time step's improvement in accuracy is not realized until above $7000 \mathrm{~Hz}$, and as will be discussed in section 4.2, the models could not accurately predict to frequencies that high.

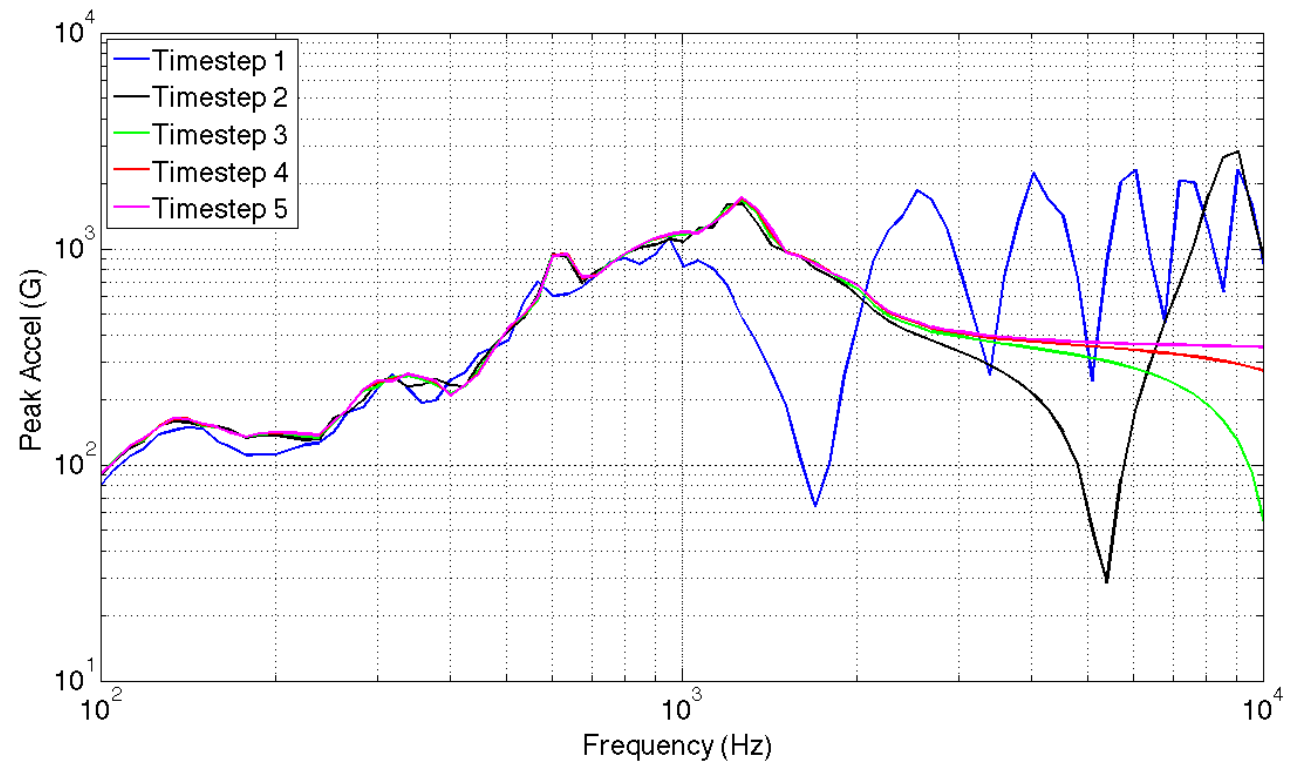

Figure 4.3 Time step study.

After concluding the time step study, a grid refinement study was necessary. All analyses from this point forward were carried out with the fourth time step size. The initial attempt used the coarsest mesh that Simulation would allow. An adequately fine mesh was defined as the mesh that stopped making major changes to the SRS compared to the previous mesh. Table 4.2 presents the progression of meshes and the maximum change in SRS between two successive meshes in $\mathrm{dB}$. Note that more nodes indicates a finer mesh. 
Table 4.2 Grid refinement study.

\begin{tabular}{|l|c|c|c|c|c|c|c|}
\hline Attempt & 1 & 2 & 3 & 4 & 5 & 6 & 7 \\
\hline \# of Nodes & 4561 & 4944 & 6010 & 6731 & 7338 & 8682 & 9664 \\
\hline$\Delta$ SSRS (dB) & & 5.5 & 7.6 & 4.4 & 5.9 & 4.4 & 2.1 \\
\hline
\end{tabular}

Figure 4.4 presents the results of the grid refinement study. The seven grids did not exhibit as drastic of differences as did the time step study, but they did not agree well across half of the x-axis either. The general shape was the same for all meshes, but each mesh introduced its own set of peaks and valleys into the SRS. While the sixth and seventh meshes still had some differences, they were minor and the two curves agreed well at most frequencies.

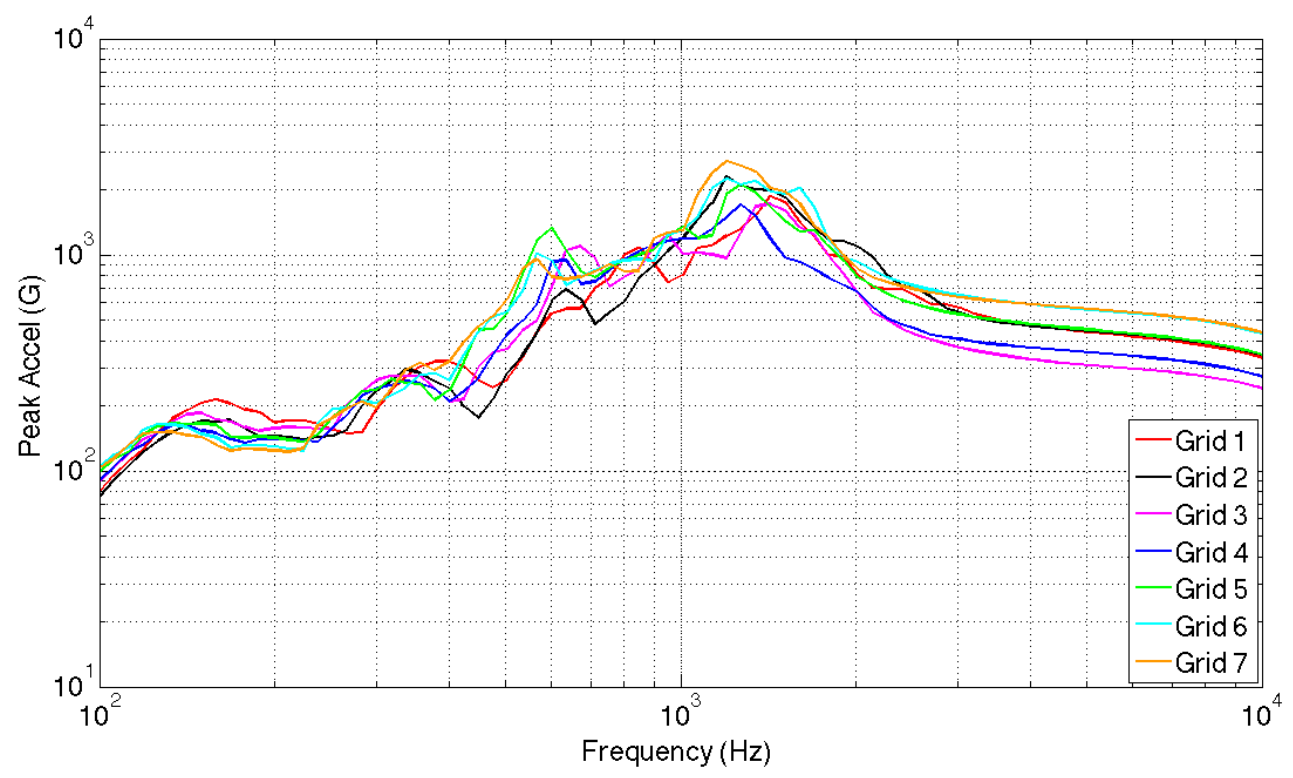

Figure 4.4 Grid refinement study.

With a maximum difference of $2.1 \mathrm{~dB}$ between the two curves, the author felt comfortable choosing the sixth mesh. While the seventh mesh was likely slightly more accurate, the author was more concerned with general curve shape and did not think that 
the minor increase in accuracy was worth the large increase in computation time. With the basic analysis parameters chosen, the model could be compared to test data

\subsection{Correlation to Test Data}

The first analysis sought to replicate the results of a test with nothing mounted to the aluminum plate other than the accelerometers. This test was the successful attempt to meet the SRS requirement from section 3.11 (note that the author wanted to meet the requirement before the Test-POD testing actually began to get a good starting point) with no loading on the aluminum plate. That requirement is still proprietary and the test data is similar to that requirement, so it cannot be displayed here. Instead of comparing the test SRS curve to the FEA SRS curve, the same type of graph will be used as was used in section 3.11. The y-axis displays the $\mathrm{dB}$ difference between the test data and the FEA data. A positive value indicates that the FEA SRS was larger in magnitude than the test curve while a negative value indicates the opposite. A value of $0 \mathrm{~dB}$ signifies a perfect match between the two curves. Figure 4.5 compares the test data and the FEA data.

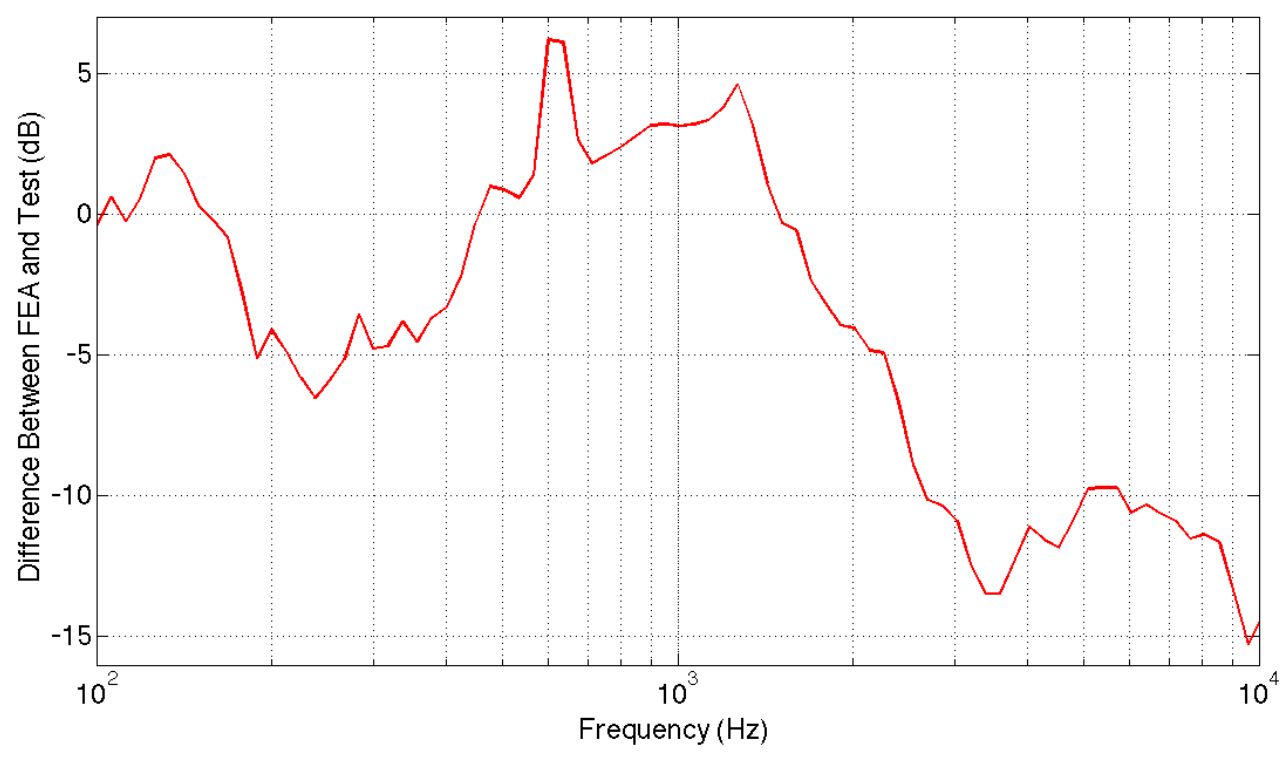

Figure 4.5 FEA of unloaded test that met SRS requirement. 
Clearly the FEA curve did not replicate the test data perfectly. This is especially true beyond $2000 \mathrm{~Hz}$, with differences approaching $15 \mathrm{~dB}$. This was to be expected however, as other simulations have had difficulty matching data beyond $3000 \mathrm{~Hz}$. [30] The author was comfortable with differences less than $5 \mathrm{~dB}$, as that corresponds to approximately half of the tolerance band of the actual requirement. This FEA was deemed a success due to its being within $5 \mathrm{~dB}$ for most of the frequency range from 100 to $2000 \mathrm{~Hz}$.

The second analysis attempted to match the results shown in Figure 3.30, which was the successful out of plane test with the Test-POD mounted to the apparatus. This setup was modeled as a point mass corresponding to the Test-POD's mass that was mounted to a flat interface plate, which was mounted to the aluminum plate. The comparison is shown in Figure 4.6.

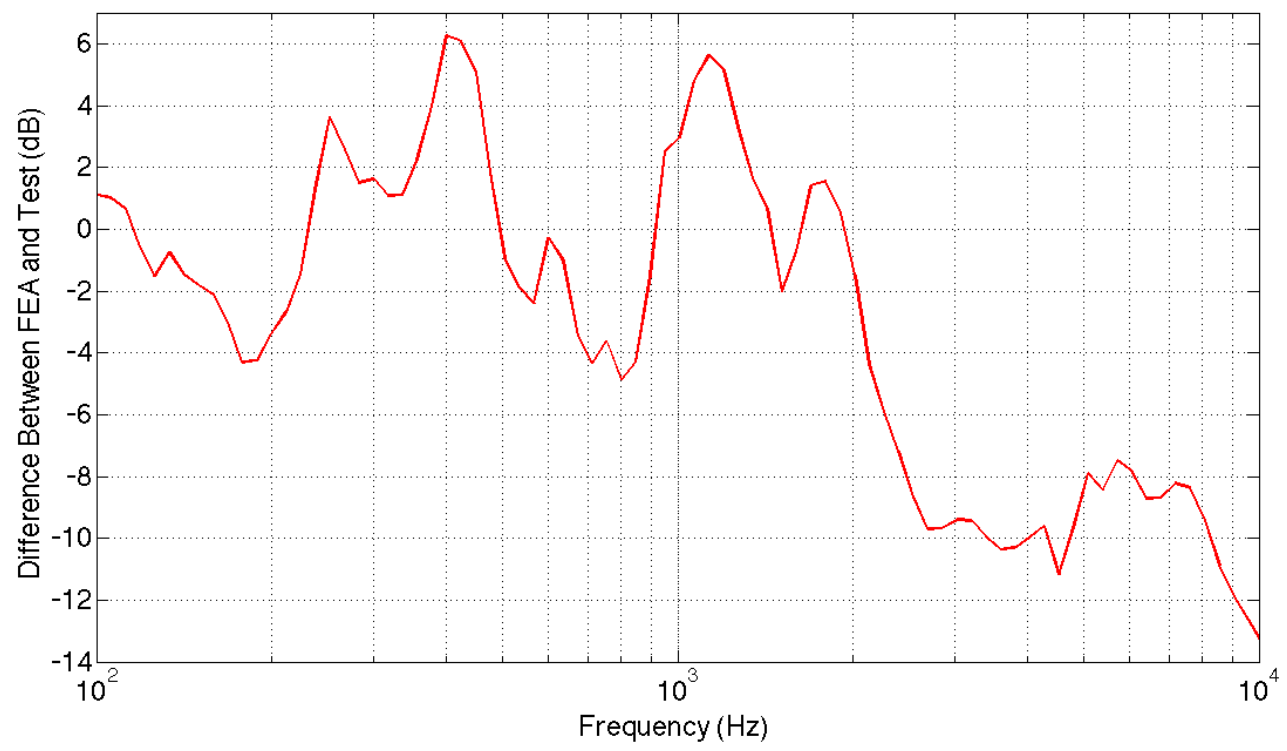

Figure 4.6 FEA of Test-POD test that met SRS requirement.

Again the correlation is far from perfect, especially for the frequencies greater than $2000 \mathrm{~Hz}$. The majority of the frequencies lower than $2000 \mathrm{~Hz}$ met the author's 
desire to have differences less than $5 \mathrm{~dB}$. It should be noted that the author never experienced issues with the out of plane testing not generating large enough magnitudes in the high frequencies, which is another reason that the lack of high frequency correlation was deemed acceptable.

The third analysis was with the L-shaped interface mounted to the aluminum plate as shown in Figure 4.2. That interface has not been manufactured or tested yet, so this analysis was an attempt to determine if the design would allow the requirement SRS to be met. Since there was no test data to compare the FEA to, the FEA was compared to the requirement SRS itself, as shown in Figure 4.7. The author was pleased with these results as the FEA SRS managed to stay within the required $+6 /-3 \mathrm{~dB}$ tolerance band up to about $1600 \mathrm{~Hz}$, other than a small over-test from about 500 to $600 \mathrm{~Hz}$. The analysis was encouraging enough to support the manufacturing of the new L-shaped interface.

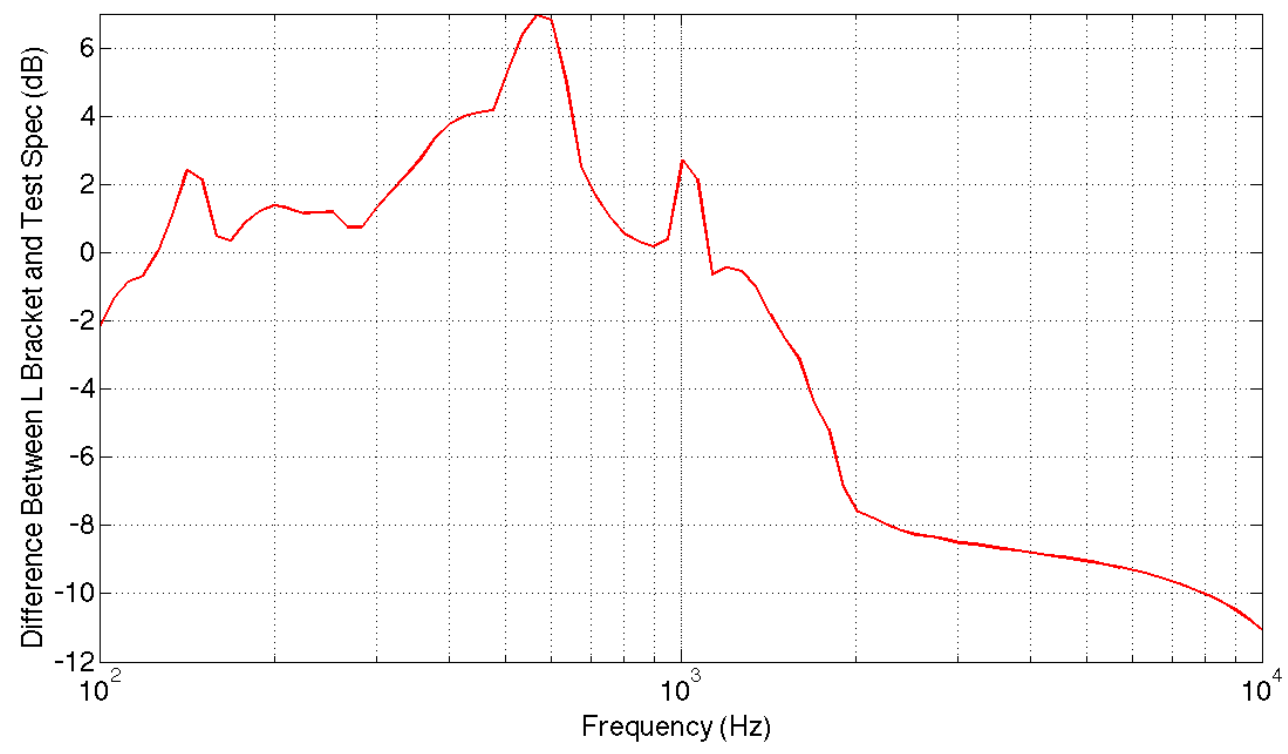

Figure 4.7 FEA of L-shaped interface compared to SRS requirement. 


\section{CONCLUSION}

\subsection{Apparatus Performance}

At the beginning of this project, the data acquisition system and overall apparatus were in place but both were lacking some key features that would allow industry-quality shock testing to be performed. A new DAQ was selected that was capable of meeting the calculated sample rate, and an analog anti-aliasing filter was added into the system. With data acquisition deemed adequate, attention was turned to the physical shock table apparatus. It was anchored to the floor to prevent movement during testing. Repeatability plates were made to provide a rigid stop against which the pendulum arm could be pulled prior to release. A new hammer was developed with the ability to change the mass added to the hammer. At that point the shock table was ready to begin testing.

Several parameters were investigated to discover their affect on the resulting Shock Response Spectrum. Increasing the release angle behaved as expected, creating a uniform (on the $\mathrm{dB}$ scale) increase in the SRS across all frequencies. Increasing the hammer mass essentially performed as expected, although in smaller magnitudes than previously hoped. It increased the SRS magnitude under about $300 \mathrm{~Hz}$ and left the rest of the frequencies largely unchanged. Increasing the diameter of the impacting hammer head did not result in the anticipated change. It did affect the SRS magnitudes in frequencies above $1000 \mathrm{~Hz}$, as expected, but the direction of the change (increase or decrease) did not remain consistent across the affected frequency range. Changing the hammer head material did result in the expected change, with harder materials creating larger magnitudes in frequencies above $1000 \mathrm{~Hz}$. Inserting a mechanical filter between the hammer head and the point of impact led to the most consistent change in SRS shape. 
A magnitude decrease in the frequencies above $1500 \mathrm{~Hz}$ could be expected. Moving the accelerometers around with respect to the impact location did not indicate any welldefined trends, with the only substantial conclusion being that a smaller distance between the two resulted in larger SRS magnitudes and more pronounced peaks in the curves. Changing the impact location to different parts of the aluminum plate provided minor changes in the SRS peaks, but did not drastically change the shape of the curve. From this test it was concluded that the general SRS shape is a characteristic of the setup geometry, material, and boundary conditions, none of which are easily changed for this shock table apparatus. Mounting the accelerometers to an interface plate instead of a mounting block did not reduce the SRS magnitudes as much as anticipated, it instead just slightly reduced the curve's overall slope. Adding mass to the interface plate affected the SRS as expected: a nearly uniform decrease across all frequencies. The trends described above were generally similar between the in plane and out of plane impact directions, although they were less pronounced. Also, the in plane impacts created a different general SRS shape than the out of plane impacts. After the parameter behaviors were tested, equalization tests with a CubeSat Test-POD were performed with a target SRS in mind. The author noticed that the trends had less significant impacts magnitude-wise with a test piece mounted to the aluminum plate. The out of plane testing successfully met the SRS requirement. The test had to be met in all three of the Test-POD's axes, which necessitated in plane testing as well. It was unsuccessful, with the different general SRS shape and the frequent zero shifts too much to overcome.

A finite element model was developed and shown to correlate reasonably well with out of plane test data from 100 to $2000 \mathrm{~Hz}$. This frequency range of correlation was 
also seen in the literature. An analysis was carried out for a new interface plate that would allow for testing in all three of a test piece's axes to be performed with out of plane impacts. The analysis indicated that this new L-shaped interface should indeed do the job.

The shock test apparatus only met its goal in one of three axes. However, it is now capable of collecting quality data and is simple to operate, only requiring one user. It is believed that manufacturing and implementing the L-shaped interface will allow the shock test apparatus to produce the required SRS in all three axes. At the very least, Cal Poly's CubeSat program can use the shock table to test a component in one axis. The shock table can also now be used to introduce students to shock testing as performed in industry, albeit on a smaller scale.

\subsection{Future Work}

The L-shaped interface should be implemented and in fact is currently being worked on. A model is presented in Appendix B.4. The author greatly desires to provide the university with a fully capable shock test facility, and intends to test the new interface when it is completed.

There are many parameters left to be explored. Some examples include changing the sacrificial impact plate material, using drastically different materials for the hammer head (hard plastic, softer metal) and mechanical filter (rubber, high density foam), manufacturing curved instead of flat hammer heads, and attempting to change the boundary conditions (rigidly clamp a large mass to a section of the aluminum plate, for example). Additionally, more testing with the existing setup should be performed to increase the number of SRS curves in the database. As the database size increases, the 
amount of trial and error when attempting to meet a requirement should decrease. On that note, work could be done on the database as well to make it more interactive. Perhaps linking the SRS database Matlab file with the Excel file containing all of the parameters for all of the test runs could achieve this.

Several improvements could be made to the pendulum hammer assembly. It currently slides back about 1/4-inch per impact when there is extra mass on the hammer. Installing rails on the ground that the base of the hammer frame slides under and attaches to is one way of solving this problem. Even with a rigid stop to pull the hammer arm against prior to release, there is some variability in the drop. A telescoping arm could be attached to the base of the pendulum frame that supports the hammer arm at the desired height. When the user was ready, the support arm would be pulled out from under the hammer arm, thus initiating the impact. The shock table is currently functional, but with these and other improvements its quality, ease of use, and range of abilities could be increased further. 


\section{REFERENCES}

[1] Filippi, Enrico. "Pyroshock Simulation Using the Alcatel ETCA Test Facility." Research Gate. Launch Vehicle Vibrations, France, Toulouse. 14-16 Dec. 1999. Web.

[2] Doody, Dave. "Basics of Space Flight." NASA. NASA, 27 July 2015. Web.

[3] Moening, C.J. "Pyrotechnic Shock Flight Failures." IES Pyrotechnic Shock Tutorial Program, 31st ATM, Institute of Environmental Sciences. April-May 1985. Web.

[4] Gaberson, Howard A. "Shock Severity Estimation." Sound and Vibration Jan. 2012. Print.

[5] "Problems in high-shock measurement." Technical report. Endevco. Web.

[6] Bateman, Vesta I.; Harry Himelblau; and Ronald Merritt. "Validation of Pyroshock Data." Sound and Vibration Mar. 2012. Print.

[7] Bateman, Vesta I. "Pyroshock Testing Update." Sound and Vibration Apr. 2008. Print.

[8] Chu, A. S. "Zeroshift of Piezoelectric Accelerometers in Pyroshock Measurements." 57th Shock \& Vibration Symposium, Part I. 1987. Web.

[9] Schweickert, G. "The Dornier Shocktable - A New Facility for Shocktesting of Components." 3rd International Symposium for Environmental Testing for Space Programs. June 1997. Web.

[10] “Mechanical Shock Facility”. Sandia National Laboratories. 2005. Web.

[11] "Pyrotechnical Shock." Technical Services Laboratory. MGA Research Corporation. Web.

[12] Sutra, M.; Didier Mesnier; Alain Berlioz; and Bertrand Combes. "Development of A Simulation Process of the Behaviour of Space Equipment Subjected to Pyrotechnic Shocks, Based on Characterization and Environmental Tests." European Conference on Spacecraft Structures, Materials and Mechanical Testing. May 2005. Web.

[13] Jonsson, Martin. Development of a Shock Test Facility for Qualification of Space Equipment. Chalmers University of Technology. Chalmers Publication Library, 5 Dec. 2012. Web.

[14] Binder, Joseph; Matt McCarty; and Chris Rasmussen. "Development of a Pyrotechnic Shock Simulation Apparatus for Spacecraft Applications.” Technical report. 
California Polytechnic State University-San Luis Obispo. Web.

[15] Nitschke, Isaac; Charles Williams. "Development of a Pyrotechnic Shock Simulation Apparatus for Spacecraft Applications.” Technical report. California Polytechnic State University-San Luis Obispo. Web.

[16] "Practical understanding of key accelerometer specifications." Technical report. Endevco. Web.

[17] "Steps to selecting the right accelerometer." Technical report. Endevco. Web.

[18] "How High in Frequency Are Accelerometer Measurements Meaningful." Technical report. PCB Piezotronics. Web.

[19] "Practical considerations in using IEPE accelerometers with modern data acquisition systems.” Technical report. Endevco. Web.

[20] Engelberg, Shlomo. “Time-limited Functions Are Not Band-limited.” Digital Signal Processing. London: Springer, 2008. Web.

[21] Smith, Strether. "Shock and Vibration Data Acquisition." Harris' Shock and Vibration Handbook. 6th ed. McGraw-Hill. Print.

[22] Randall, Robert B. "Vibration Analyzers and Their Use." Harris' Shock and Vibration Handbook. 6th ed. McGraw-Hill. Print.

[23] Himelblau, Harry. Handbook for Dynamic Data Acquisition and Analysis. Institute of Environmental Sciences and Technology. Print.

[24] Proceedings of 82nd Shock and Vibration Symposium, Baltimore, Maryland, USA. 2011.

[25] Kelly, Ronald D., and George Richman. Principles and Techniques of Shock Data Analysis. Washington D.C.: United States Government Printing Office, 1971. Print.

[26] Smallwood, David O. "An Improved Recursive Formula for Calculating Shock Response Spectra.” 56th Shock \& Vibration Symposium, Part I. 1987. Web.

[27] Irvine, Tom. "An Introduction to the Shock Response Spectrum.” Technical report. Vibrationdata. Web.

[28] NASA. "GSFC-STD-7000A, General Environmental Verification Standard." NASA, Greenbelt, Maryland, 2013. Web. 
[29] United States Department of Defense. "MIL-STD-810F, Environmental Engineering Considerations and Laboratory Tests.” Jan. 2000. Web.

[30] Kiryenko, Stefan; Gaetan Piret; and Jan Kasper. ESA/ESTEC Shock Bench

Presentation. European Conference on Spacecraft Structures, Materials, \& Mechanical Testing, 12 May 2005. Web.

[31] Piersol, Allan G. Pyroshock Data Acquisition and Analysis for U/RGM-109D Payload Cover Ejection Tests. China Lake: Naval Weapons Center Technical Information Department, 1988. Print. 


\section{APPENDICES}

\section{A Operating Instructions}

The shock table is intimidating, but is actually pretty straightforward to operate. Before getting to that, the table's maintenance should be discussed. Shock has a strange tendency to loosen fasteners, and the author had to tighten bolts several times throughout the testing. It is recommended that all of the fasteners on both the shock table frame and the pendulum hammer frame be checked every 50 tests. The brackets shown below were especially prone to coming loose and should probably be checked every 25 tests. They are located at either end of the horizontal frame pieces both above and below the aluminum plate.

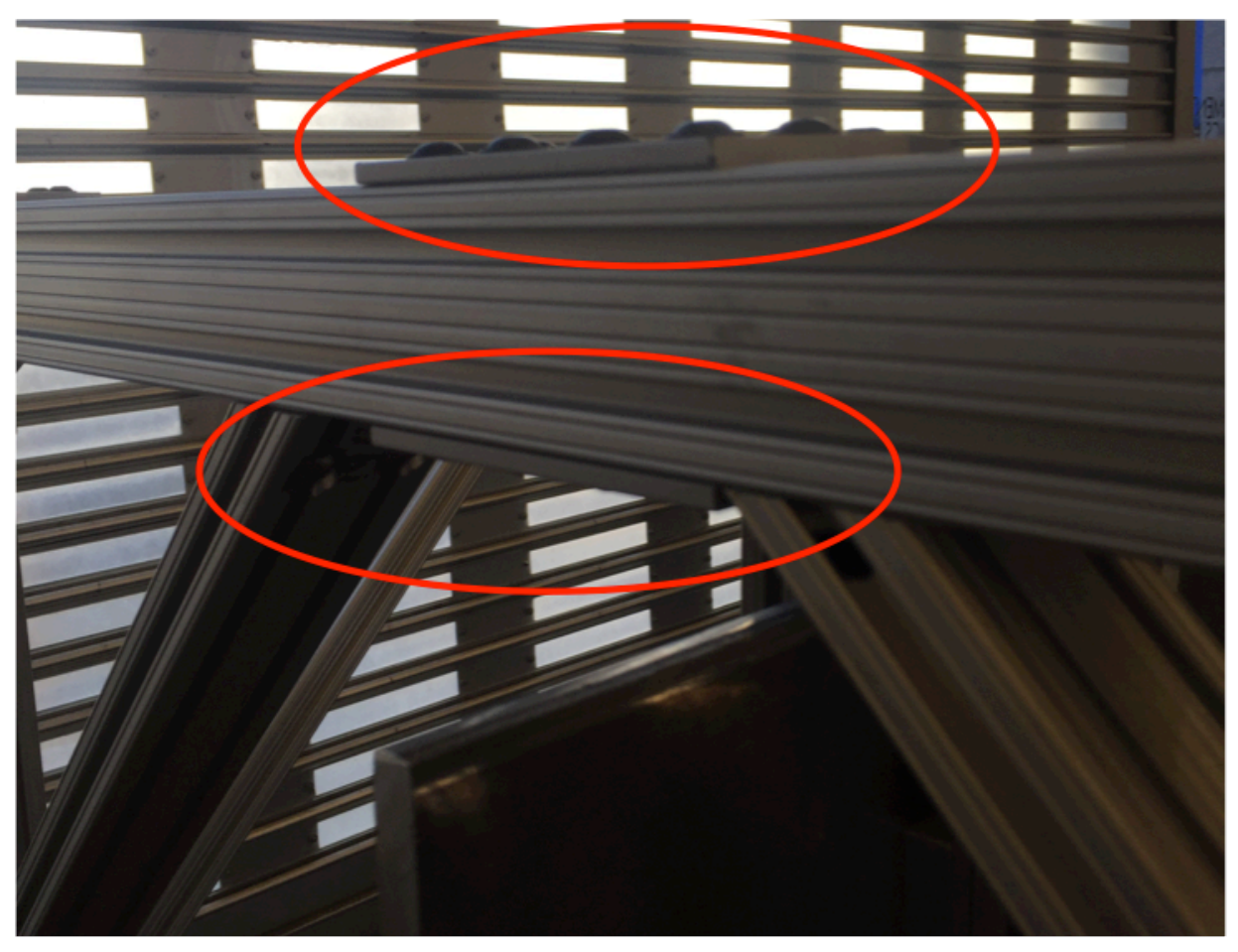

\section{A.1 Preparing Data Acquisition System}

Obviously the poorly made plastic boxes housing the filter board and the DAQ are not industry standard. The enclosures they could have come in were very expensive, 
and were not purchased, but they have sensitive components and care should be taken to not expose them to static or electromagnetic interference. The manufacturer stated that both boards are sensitive to power loss, so DO NOT PLUG/UNPLUG COMPONENTS WITH THE POWER ON. Now without further ado, instructions:

1) Mount accelerometers. If using a mounting block, it should be as close to level as possible. Mounting surface should be flat and prepared with thin layer of silicone grease. Accelerometers should be torqued to $50 \mathrm{in}-\mathrm{lb}$. Accelerometer website is here: http://www.dytran.com/Model-3200B4-Shock-Accelerometer-P2371.aspx and data sheet, technical drawing, and mounting instructions can be found here: http://www.dytran.com/Assets/PDF/3200B4_ds.pdf

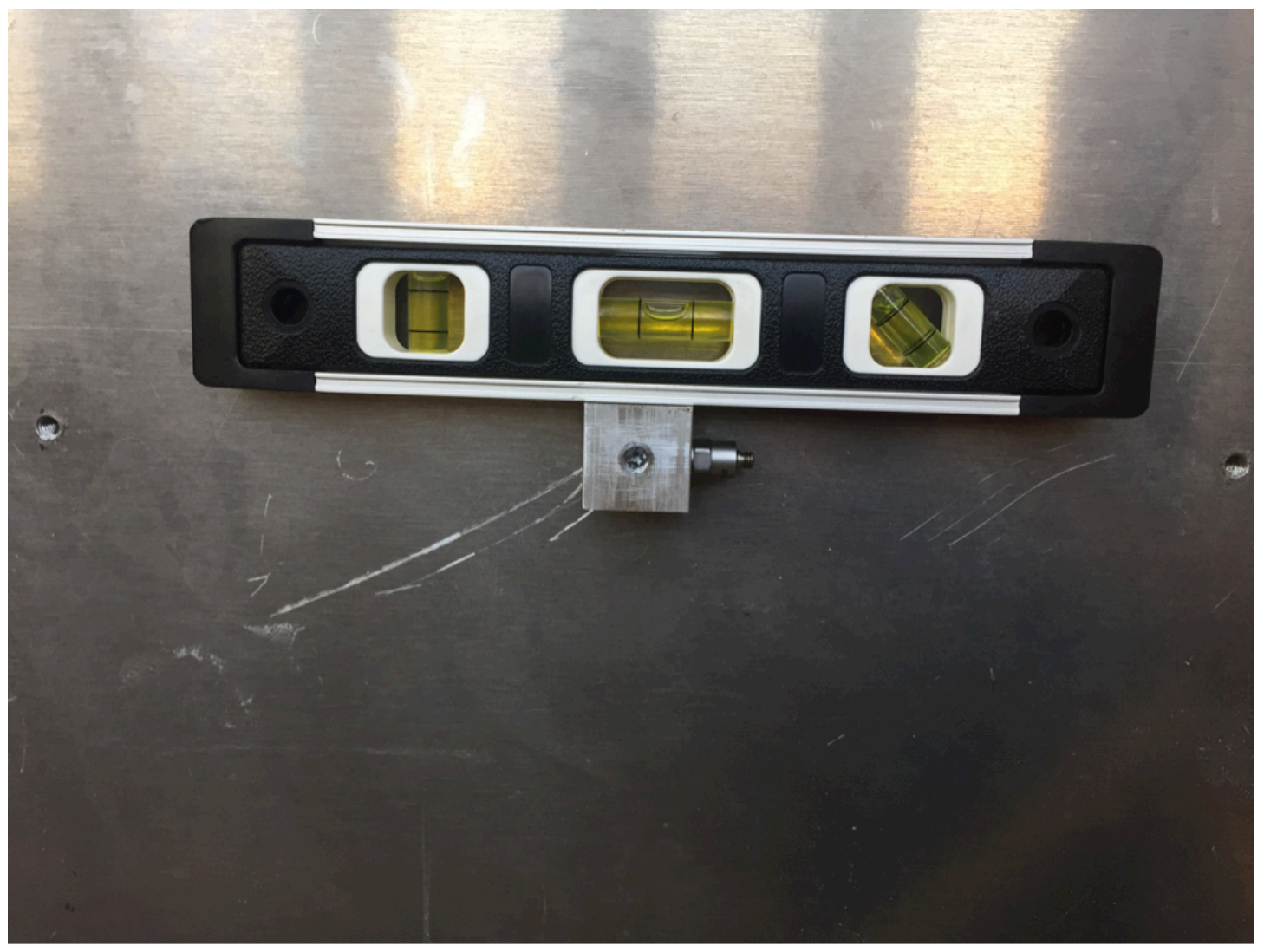


2) Attach the low noise coaxial cable (white) to the accelerometers. To avoid issues with the cable coming loose during a test, it is recommended that it be lightly tightened with needle nose pliers. Use a light grip (read: do not squeeze hard) and turn slightly past finger tight.

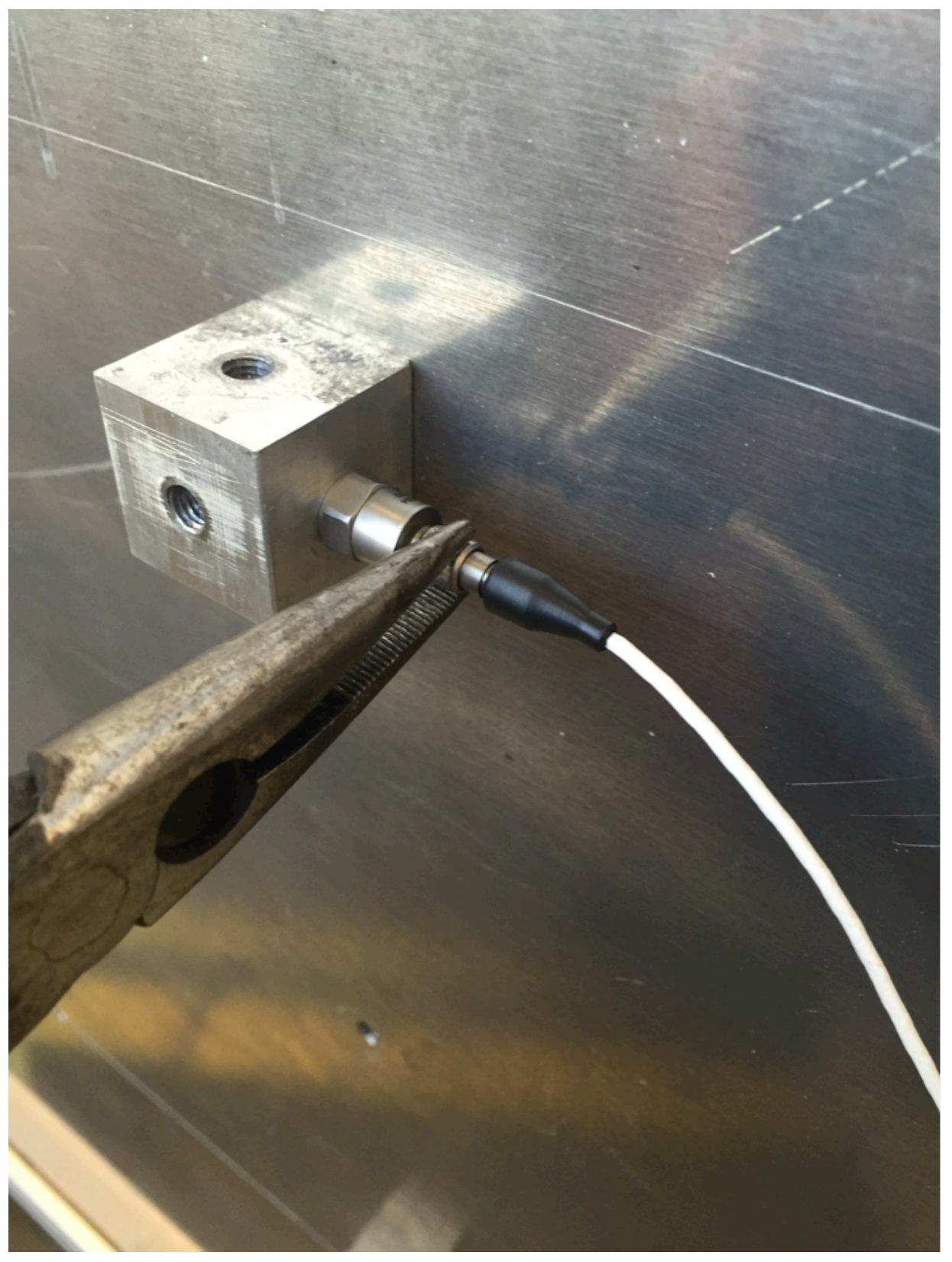


3) Connect the low noise coaxial cables (white) to the signal conditioner's ICP jack.

Note that the author always used the accelerometers in the same configuration: accelerometer 8158 in Channel 1, 8159 in Channel 2, and 8160 in Channel 4. Channel 3 did not always work. Connect the regular coaxial cables (black) to the signal conditioner's OUTPUT jack.

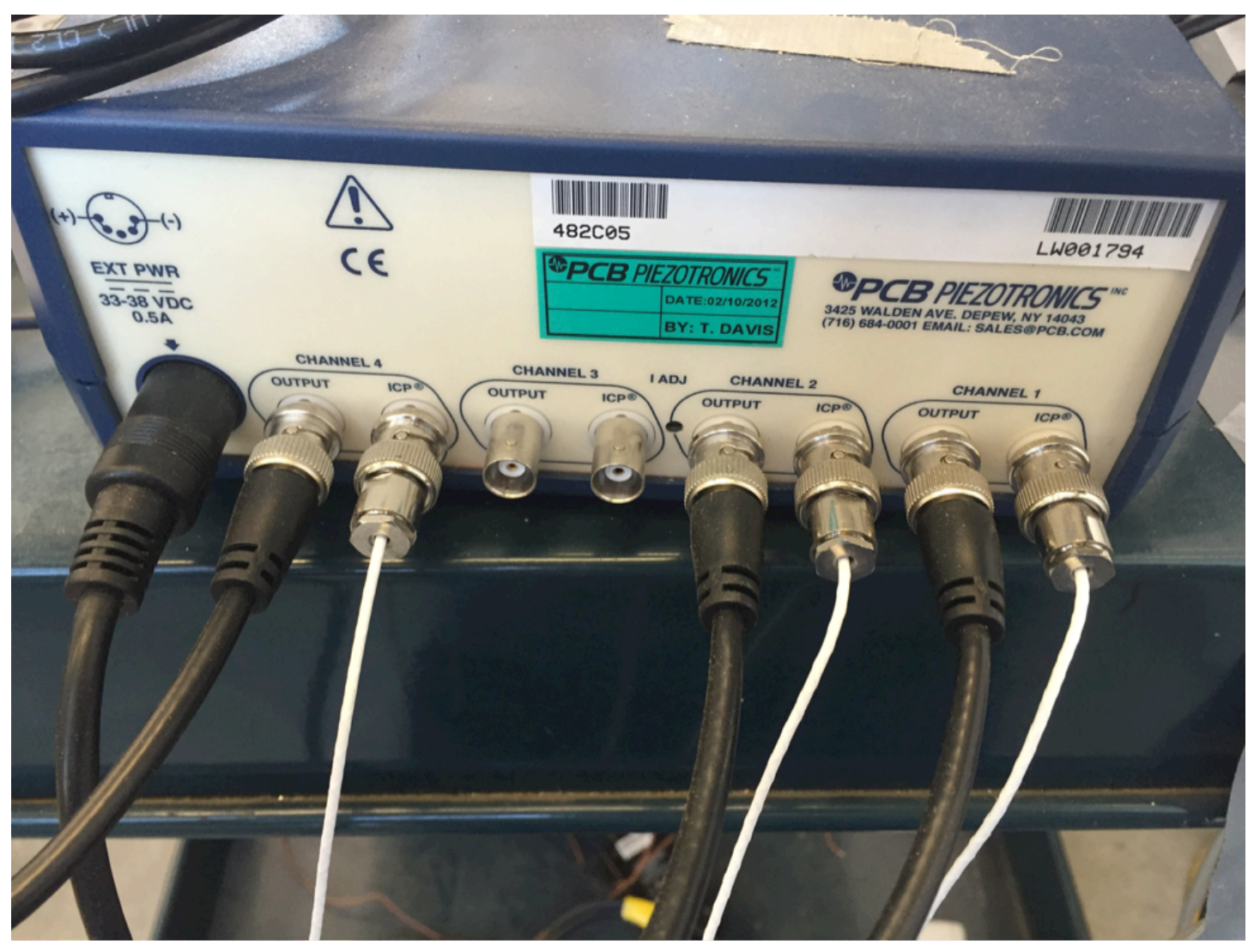


4) Turn the signal conditioner on. Wait for any overload and short lights to go out.

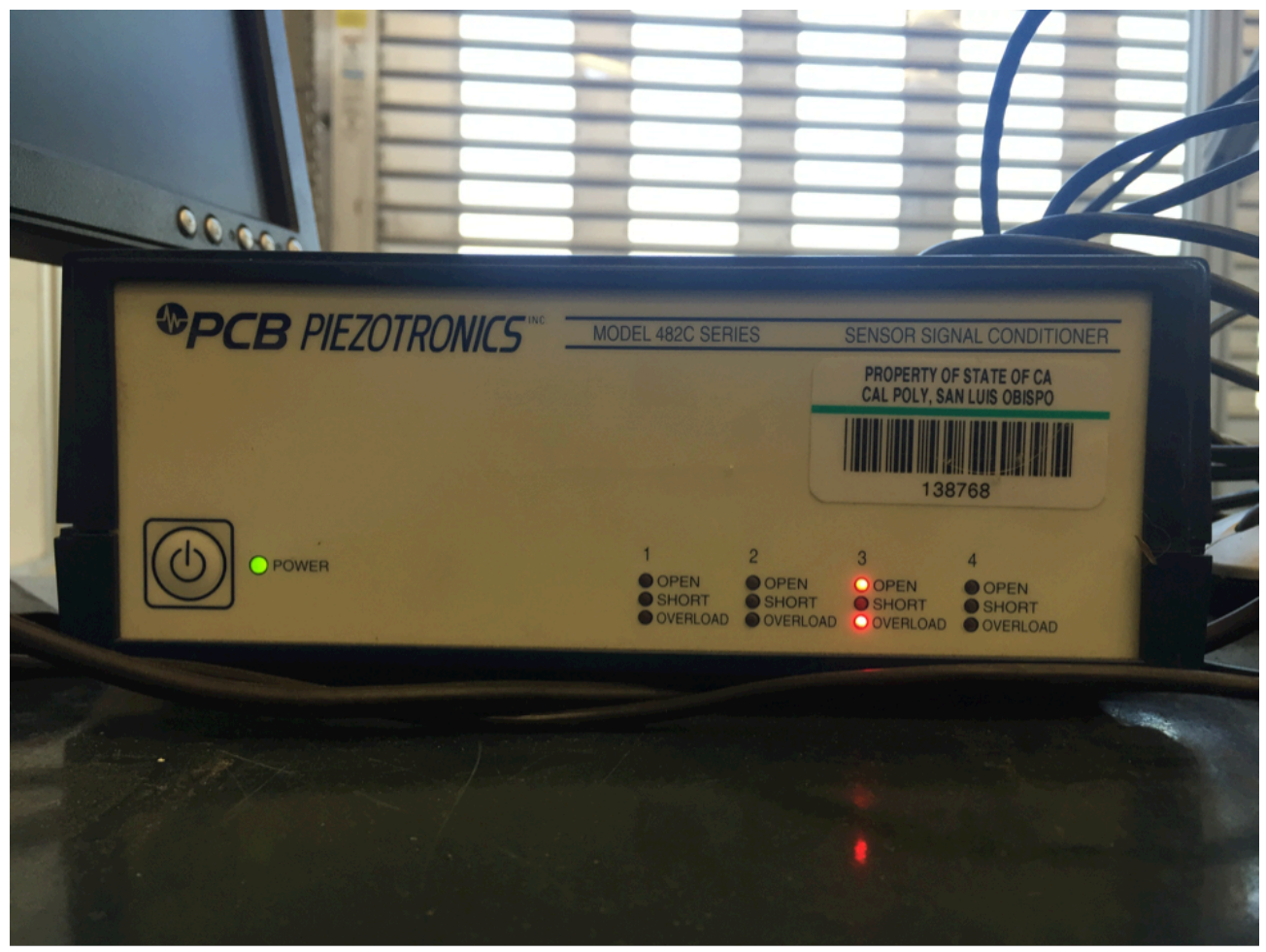

With Channels 1,2, and 4 in use, the lights should settle to the following:

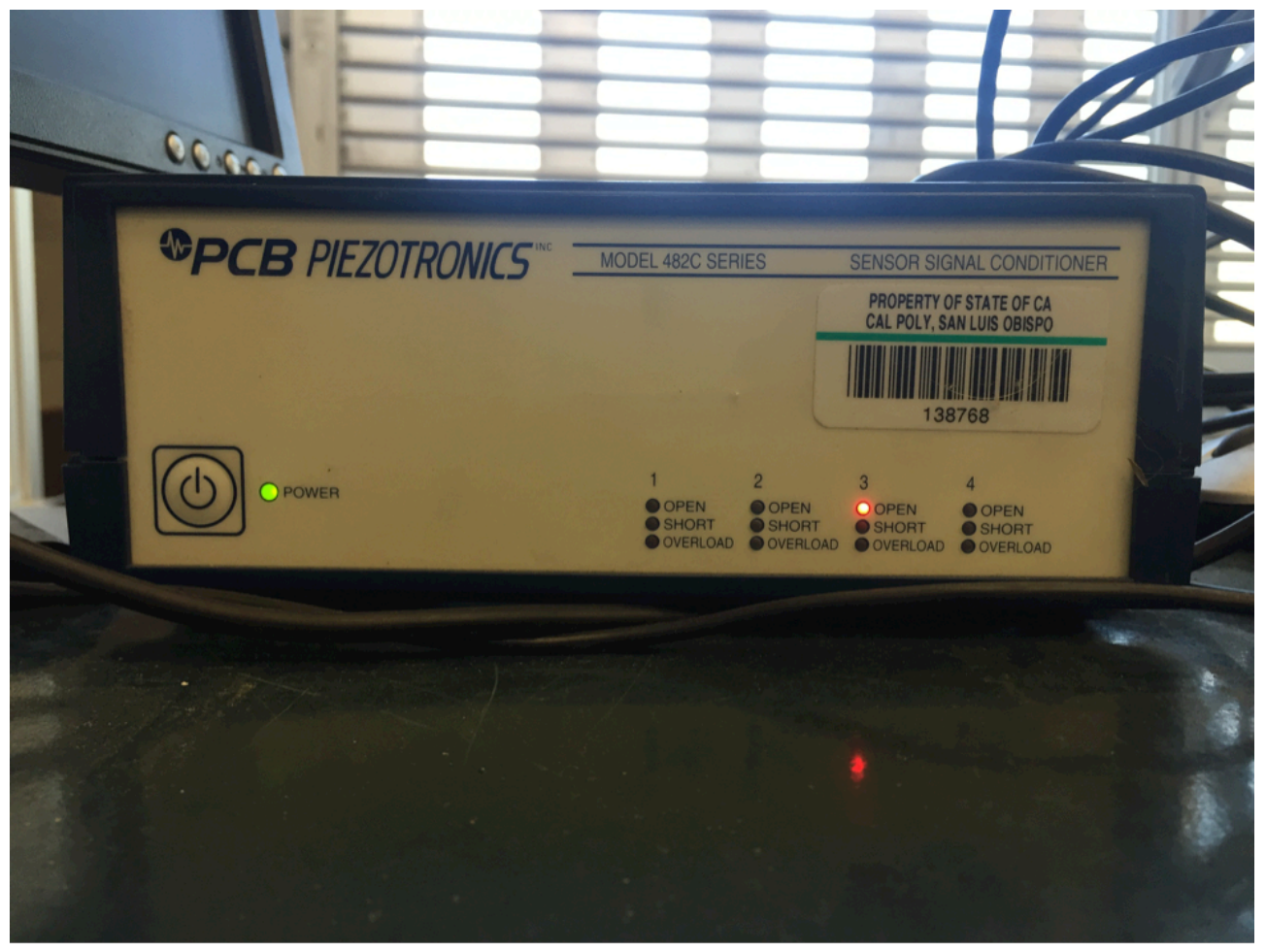


5) Attach the regular coaxial cables (black) to the filter termination board as shown.

Note that DAPStudio (Microstar's version of LabView) samples in channel groups of 4, and for whatever reason the first channel group corresponds to jacks 19 (1A), 17 (3A), and 15 (1B). The program reads jack 19 as input 1 , jack 17 as input 2 , and jack 15 as input 3 . Always connecting accelerometer 8158 to signal conditioner Channel 1 to filter board jack 19, accelerometer 8159 to Channel 2 to jack 17, and accelerometer 8160 to Channel 4 to jack 15 is a good way to keep track of the data.

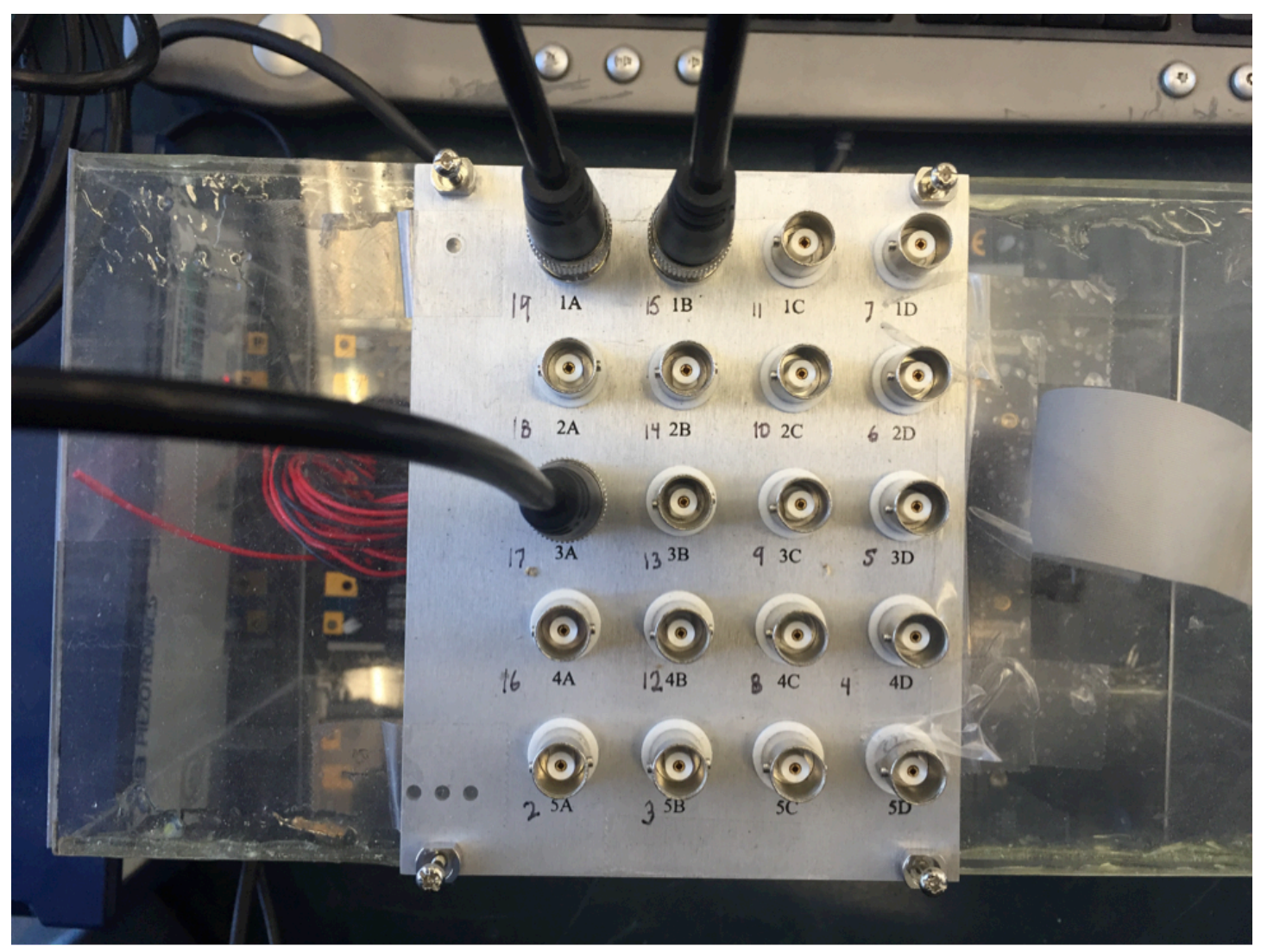


6) There should be a ribbon cable attached to the filter board inside the tall plastic box. Never unplug it. Plug the other end into the DAQ (it's actually a Microstar DAP 5380a). Do not unplug this cable with power to the system. The manufacturer implied the apocalypse would happen if it was ever unplugged with power. Do not do it.

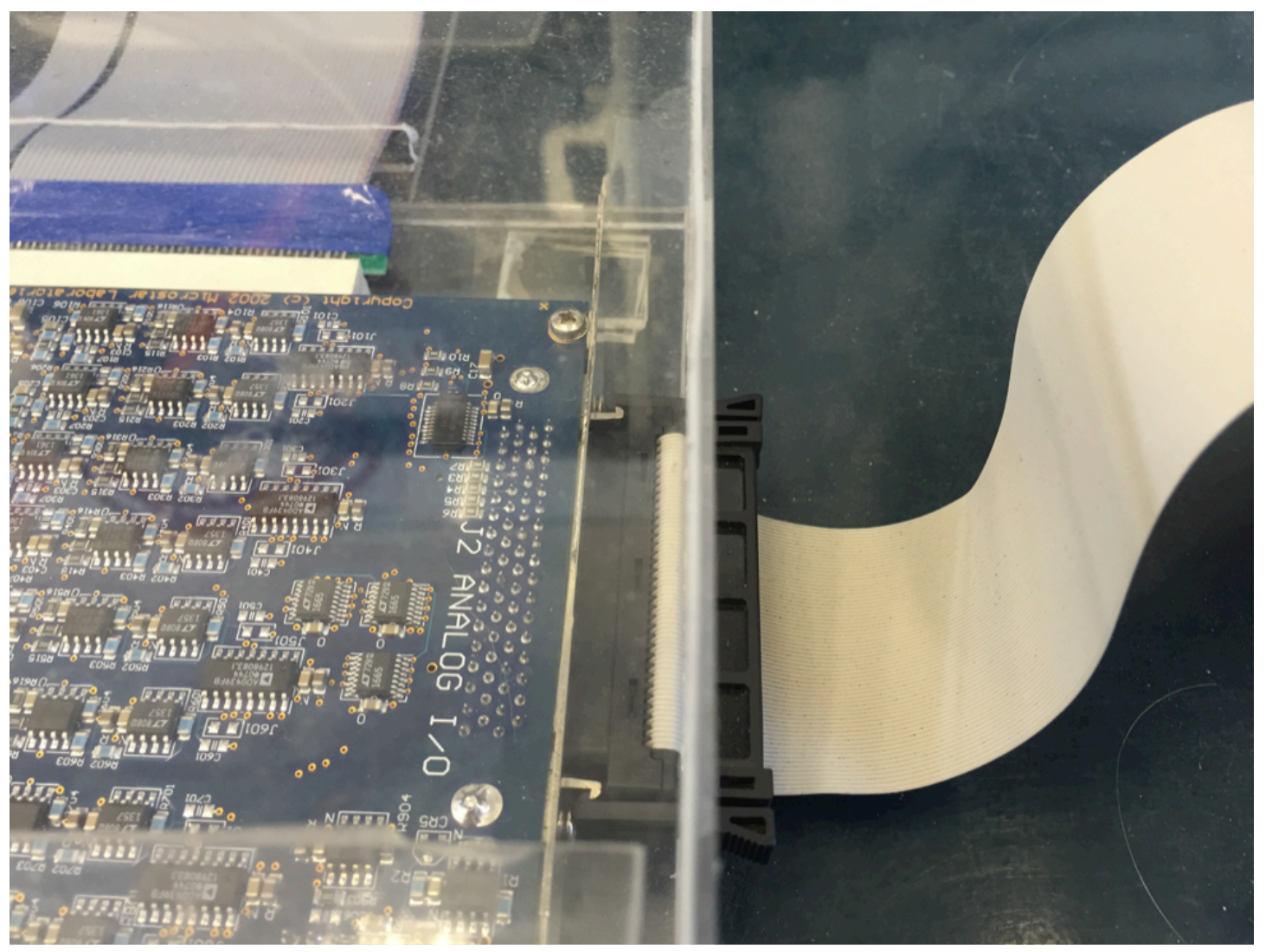

7) The DAQ should be connected to the computer's PCI slot with another ribbon cable.

If it is not, attach it now. Plug the computer in and turn it on. Note that the computer was kept unplugged when not in use because it had a strange habit of turning itself back on several hours after shutting down. This caused the DAQ's fan to run unnecessarily, which is not good. 
8) Open the DAPStudio file "Cal Poly Shock Data".

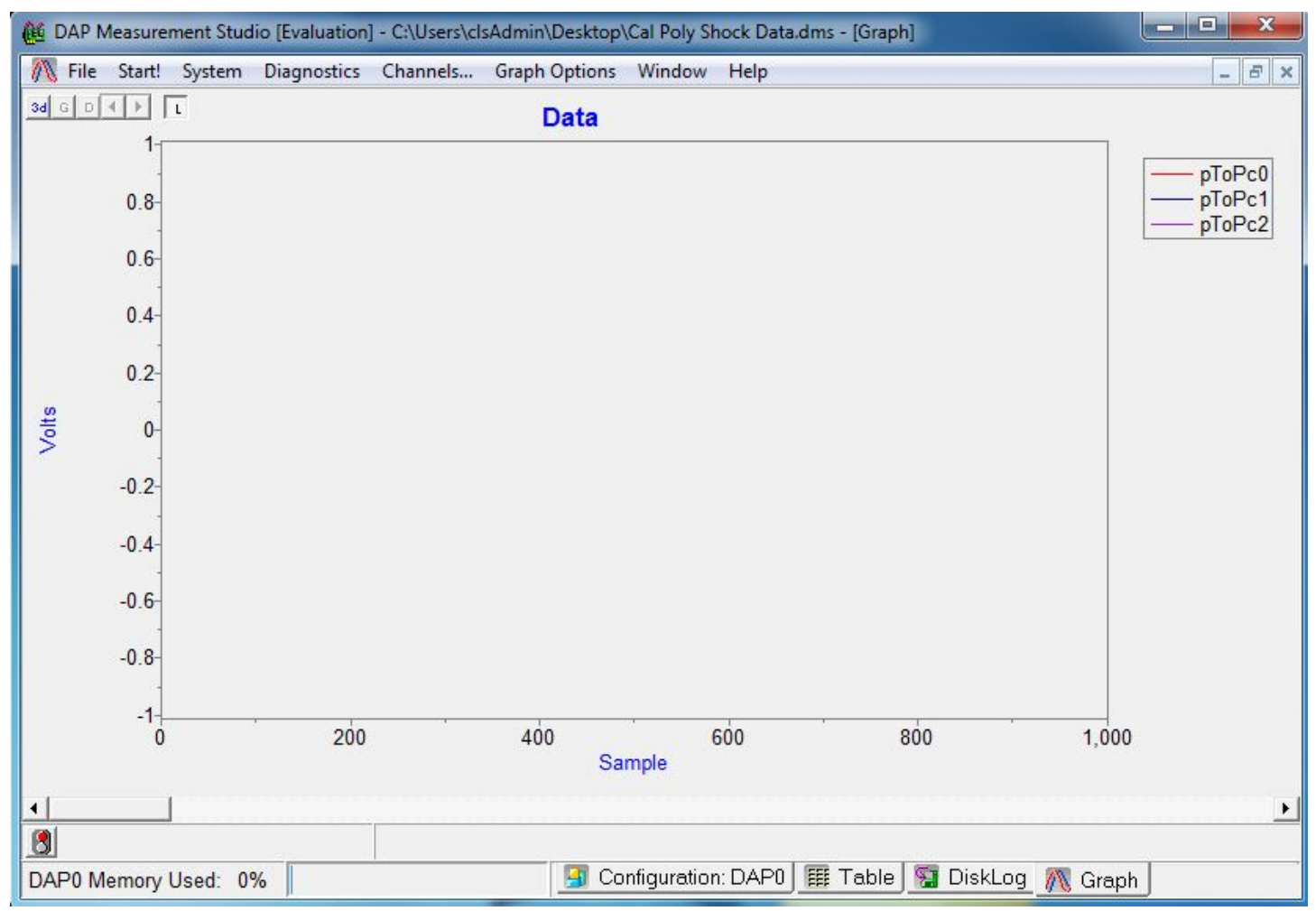


9) Click on the DiskLog tab at the bottom of the screen. The Format should be set to Text. Select a destination and file name to save the data.

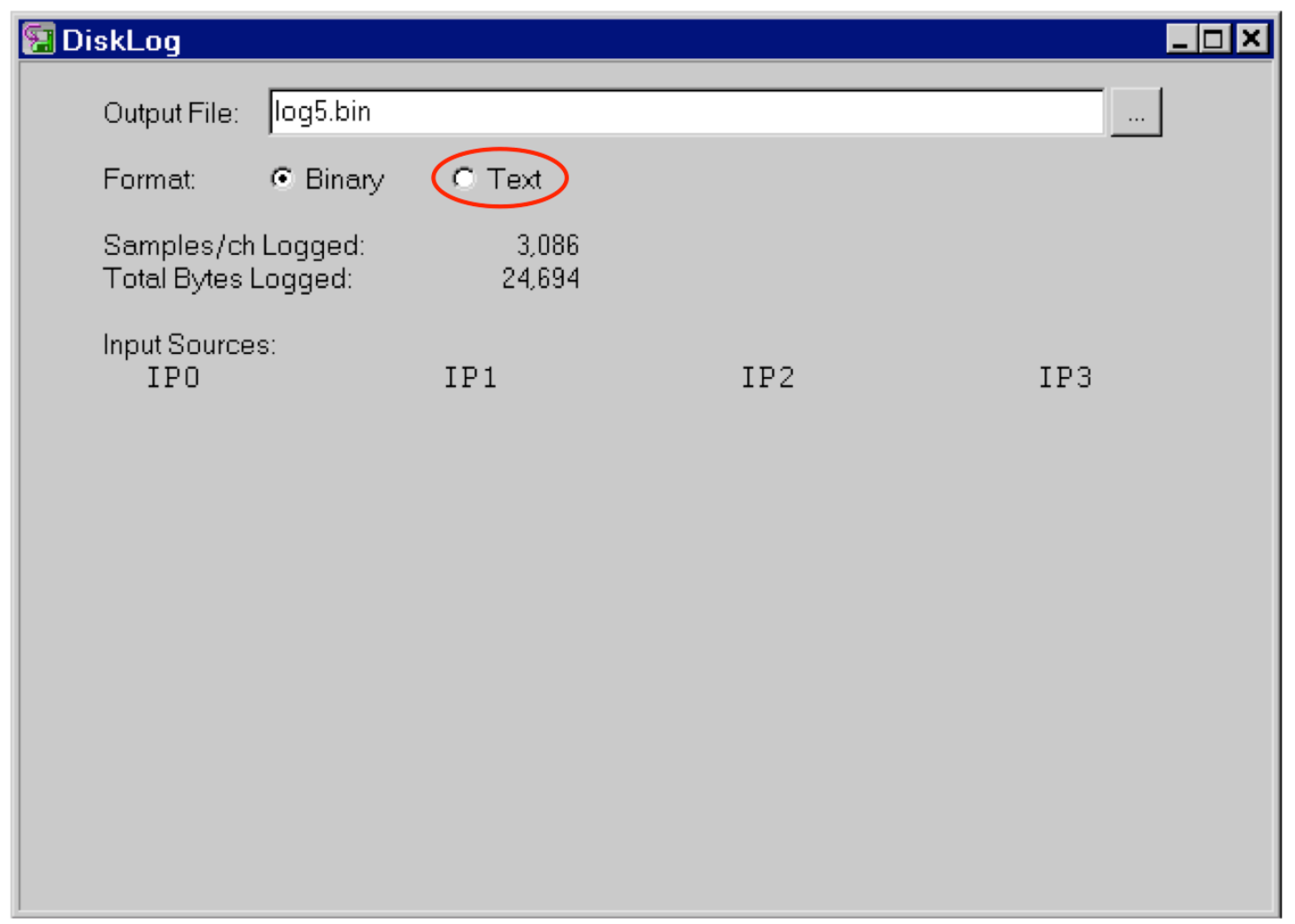

10) The system is ready to go. Click "Start" to begin collecting data. Note that the first time "Start" is clicked after the program has opened, a popup will open requesting that the DapStudio program be purchased. Click "Purchase DapStudio", then click "Continue" twice. Every time "Start" is clicked after that, "Continue" must again be clicked twice before the system will start collecting data. 

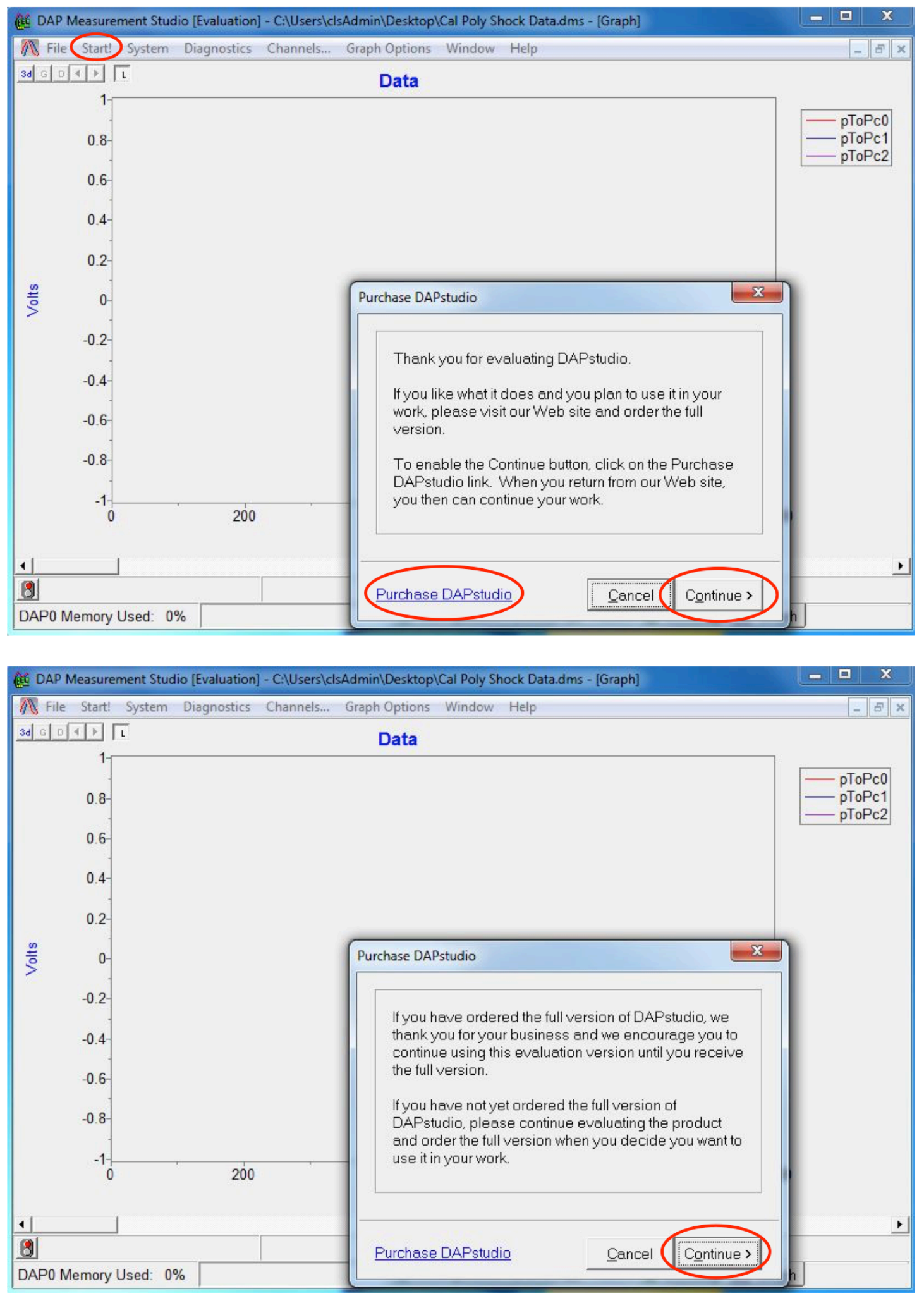

11) Once the shock test is done, click "Stop". In order for the data to actually be written

to the file specified in step 9, the DiskLog tab must be opened again. Click the "..." 
button, and the text file will be generated. Note that the program is set to "observe" the data stream without logging anything until a certain acceleration threshold is met. Once this happens, 1000 samples prior to the detected shock event are stored for each channel, and then data is captured for 0.3 seconds after the shock event. The sample rate is $600 \mathrm{kHz}$, so each channel (each accelerometer) generates 181,000 samples per test.

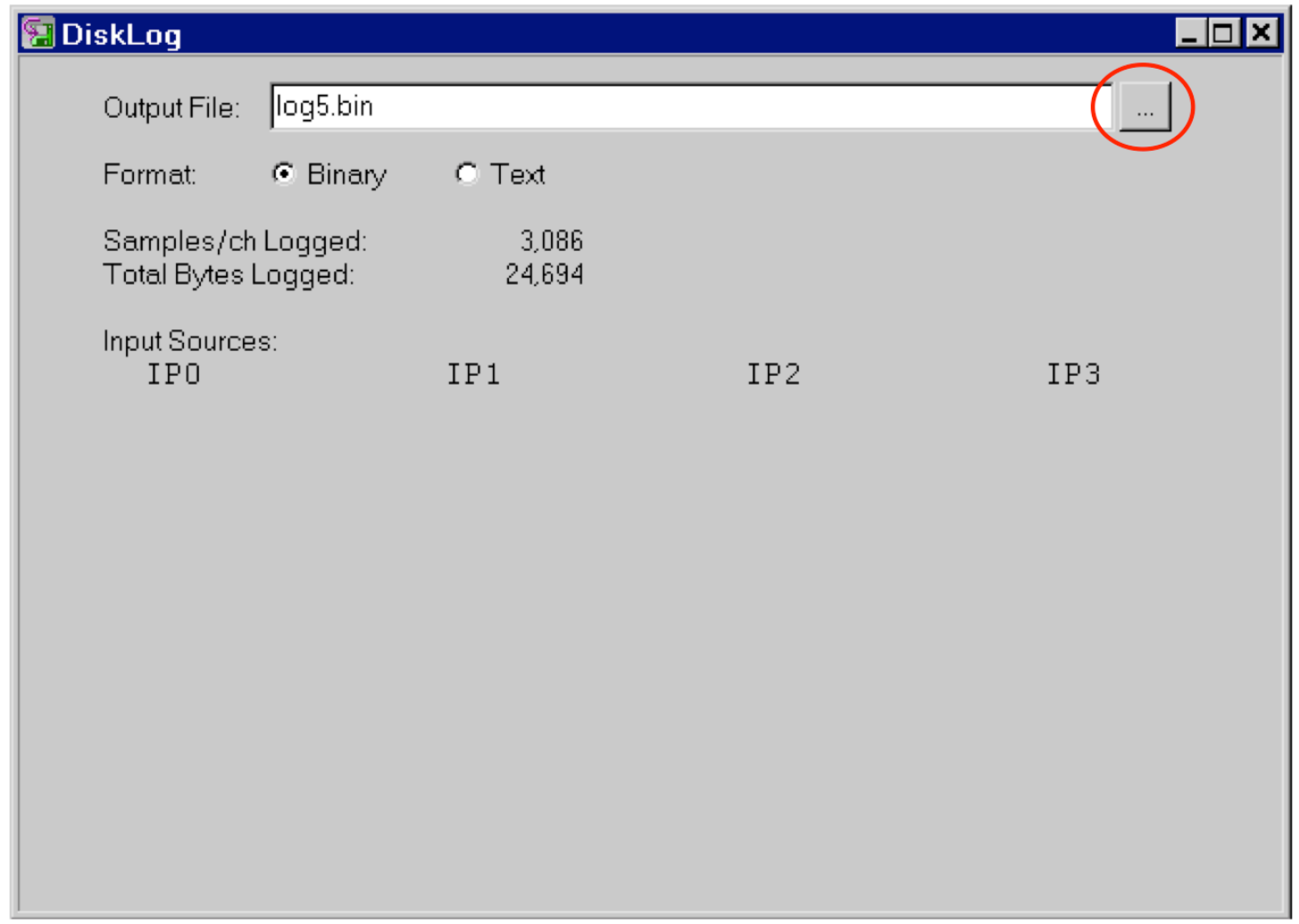

12) Success. When done, work backward from step 7 to step 1 to turn the system off. 


\section{A.2 Adjusting Shock Table}

The table has several parameters that can be changed, and most of them are easily changed. The accelerometers can be mounted in different positions. The pendulum arm can be released from different angles with the help of the repeatability plates. Pictures are included of several of the parameters. Note that they can be adjusted in any order, but the adjustments should be made prior to clicking "Start", as an accidental bump of the table could initiate data collection.

1) Add or change hammer mass. There's a threaded rod and two sets of nut and washer.

Thread the rod through the hammer and screw one washer and nut under the hammer.

Put the desired mass on the hammer and screw the other washer and nut on top.

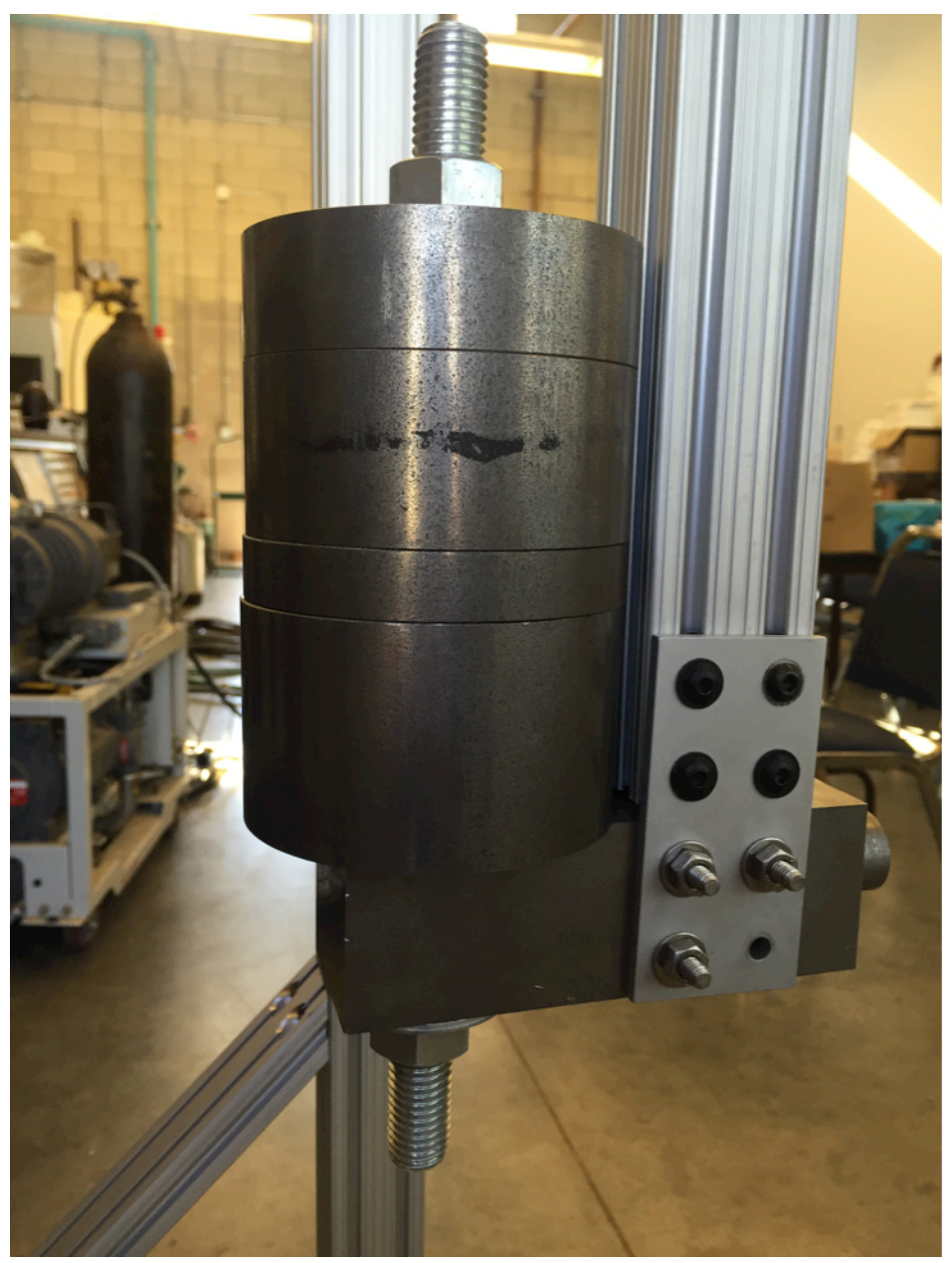


2) Change hammer head radius. Loosen the set screw with $1 / 8$-inch Allen wrench.

Remove hammer head. Insert desired hammer head. Tighten set screw.

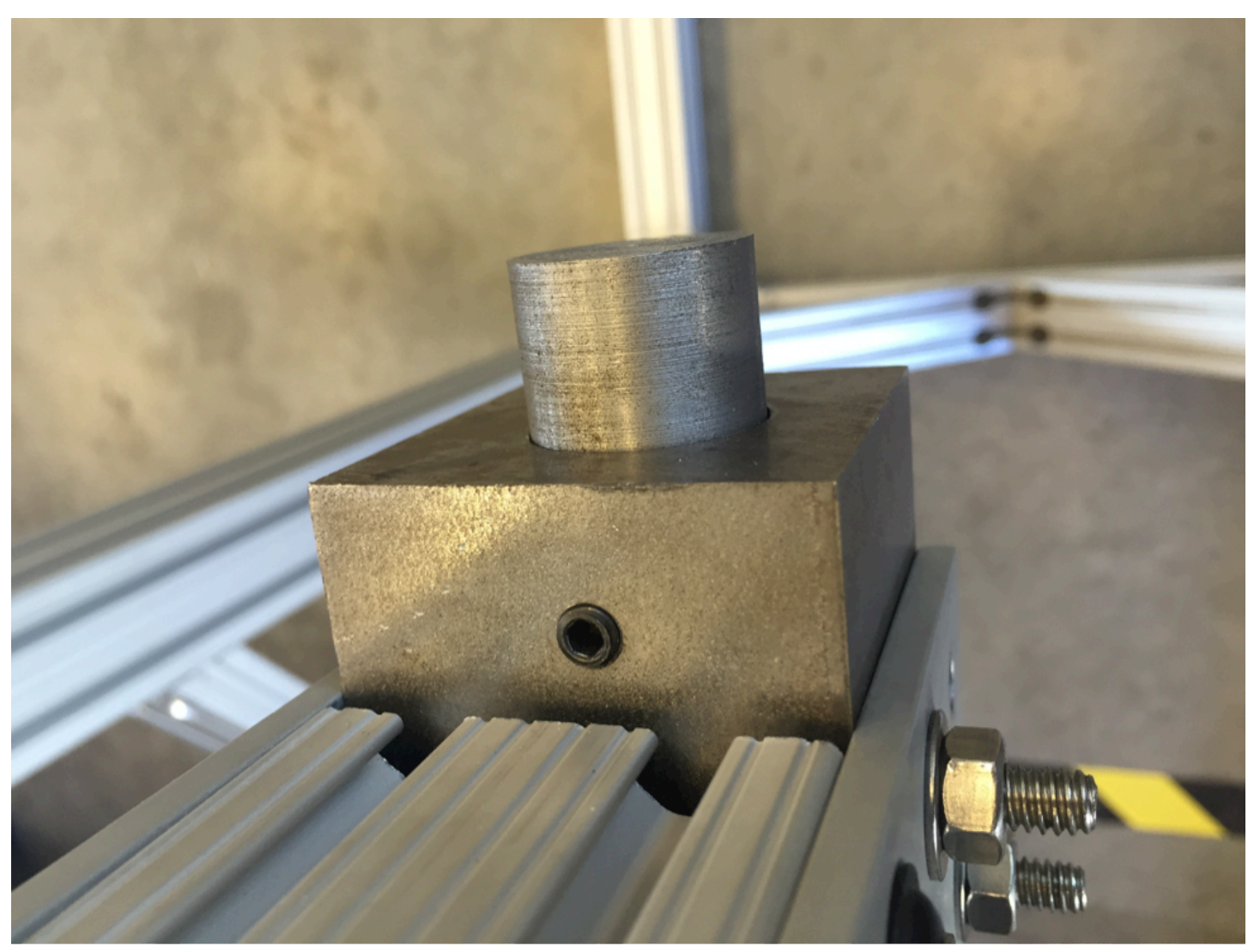


3) Change impact location. Move the entire pendulum frame. Take care to line up the frame so that the hammer head is parallel to the plate upon impact. Always impact the sacrificial plate, so move it whenever the pendulum frame is moved. The bolts shown are called Hex Button Head Cap Screws, and all fasteners like them on the table require a 5/32-inch Allen wrench.

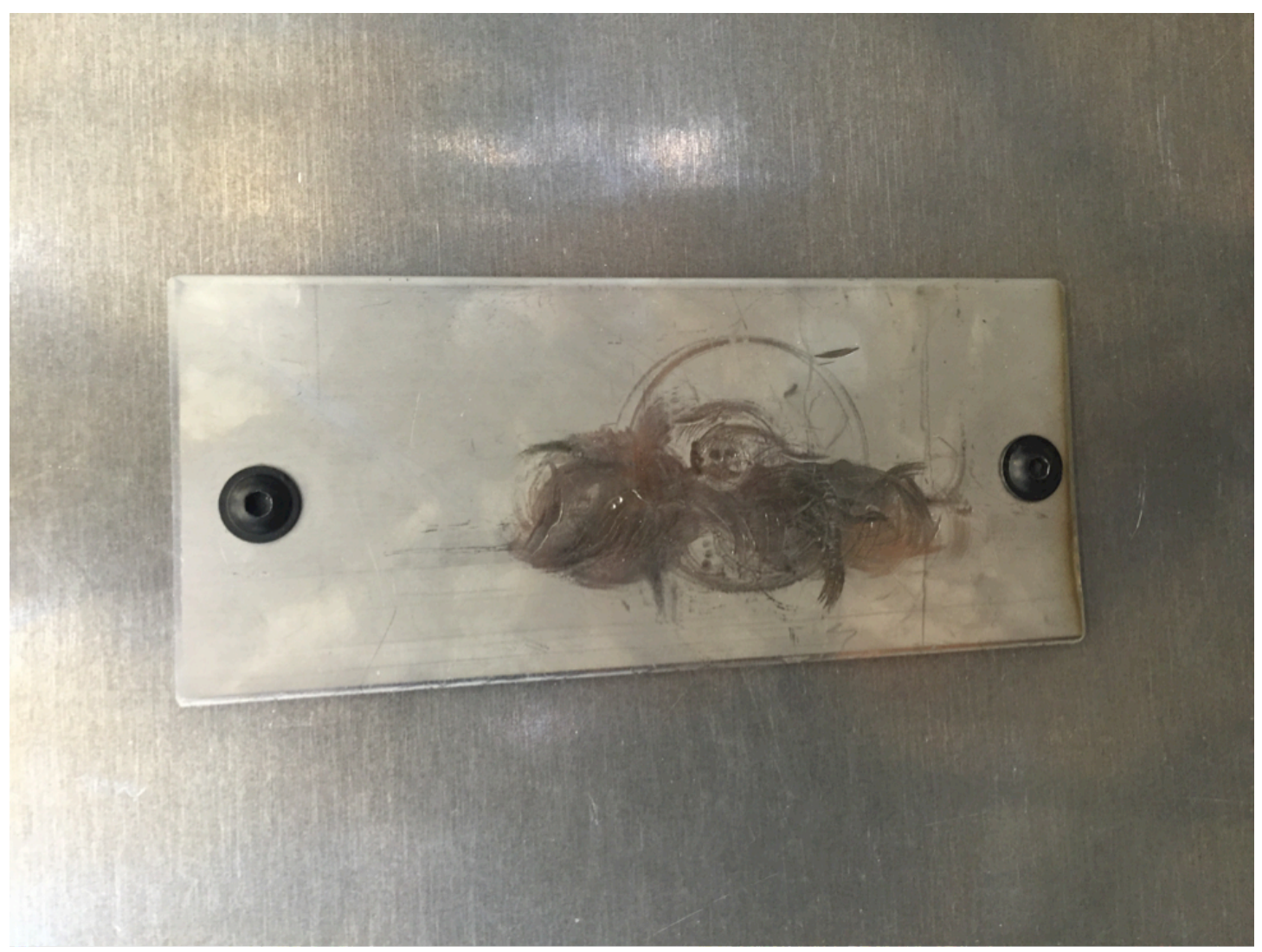


4) If the impact location is to be shifted up and down instead of side to side, the process is a bit more involved. It is recommended that this be done with no extra mass attached to the hammer. Loosen (do not unscrew all the way as it is difficult to put back together) the 4 bolts on each of the repeatability plates. A 7/16-inch socket and about a half turn should do the trick. Slide the plates down and out of the way.

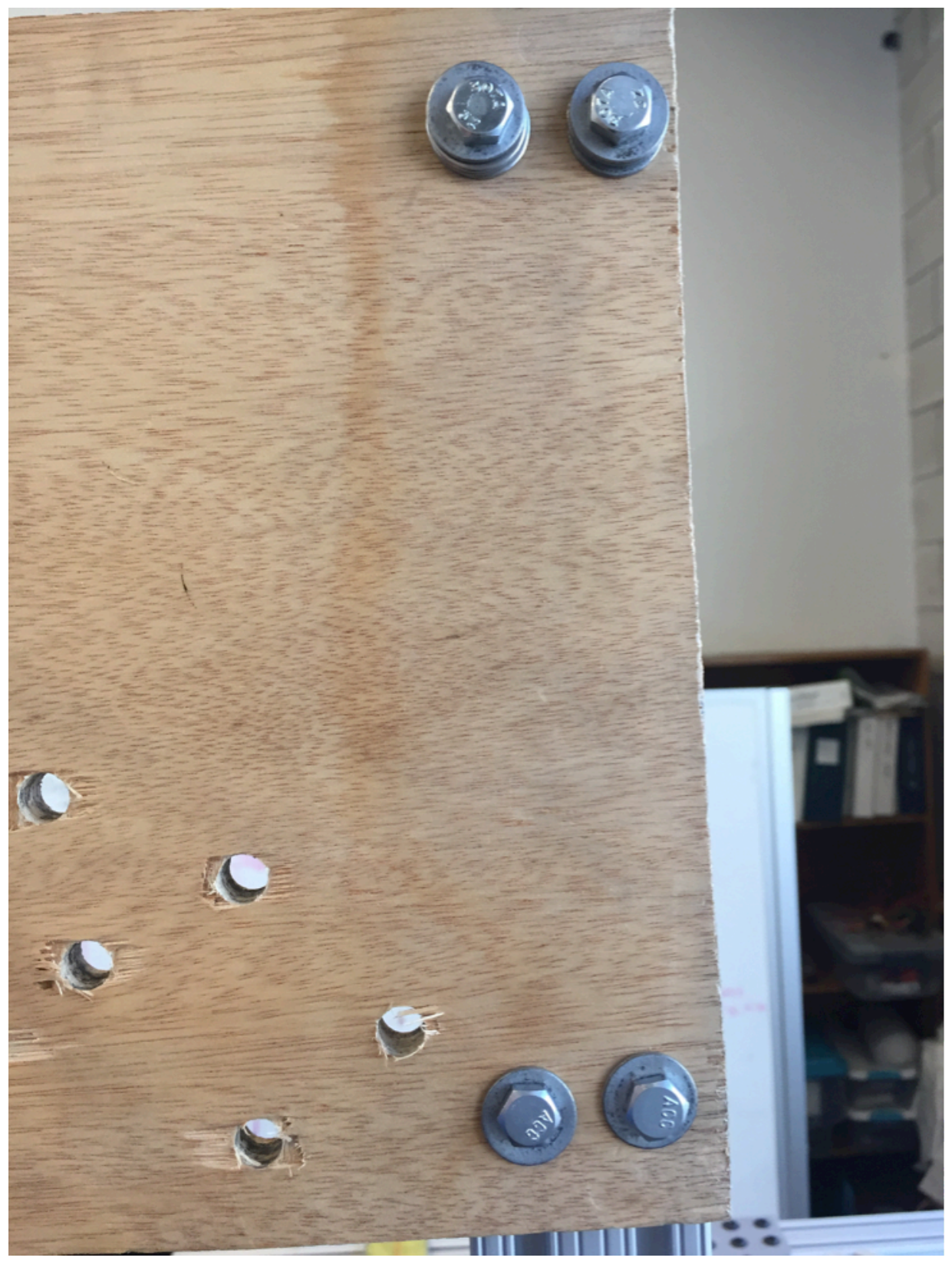

Loosen (again, do not completely remove) the 4 bolts shown. Then loosen the 4 bolts 
on the same piece on the other side of the pendulum frame. The bolts are called Socket Head Cap Screws and all fasteners like them on the table require a 3/16-inch Allen wrench.

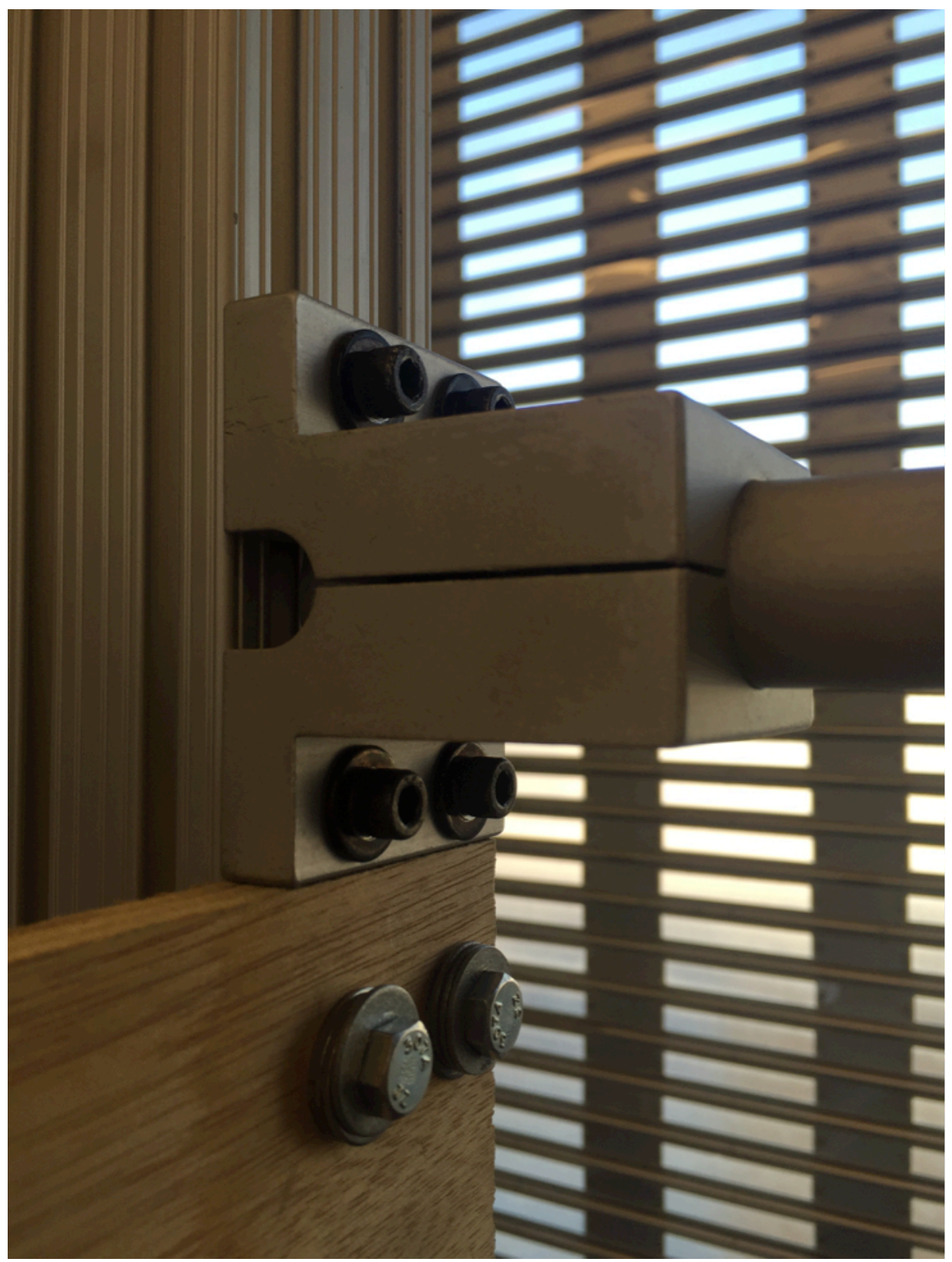

Slide the hammer arm up or down to the desired position. If it does not slide easily, check the 8 Socket Head Cap Screws that were just loosened. If any of them cannot be turned by fingers, loosen them further. Once in position, tighten the 8 Socket Head 
Cap Screws. From here it is recommended that the hammer arm be checked for level.

The proposed method is shown. Place something flat across the brackets that were just moved, and check for level. Adjust as necessary.

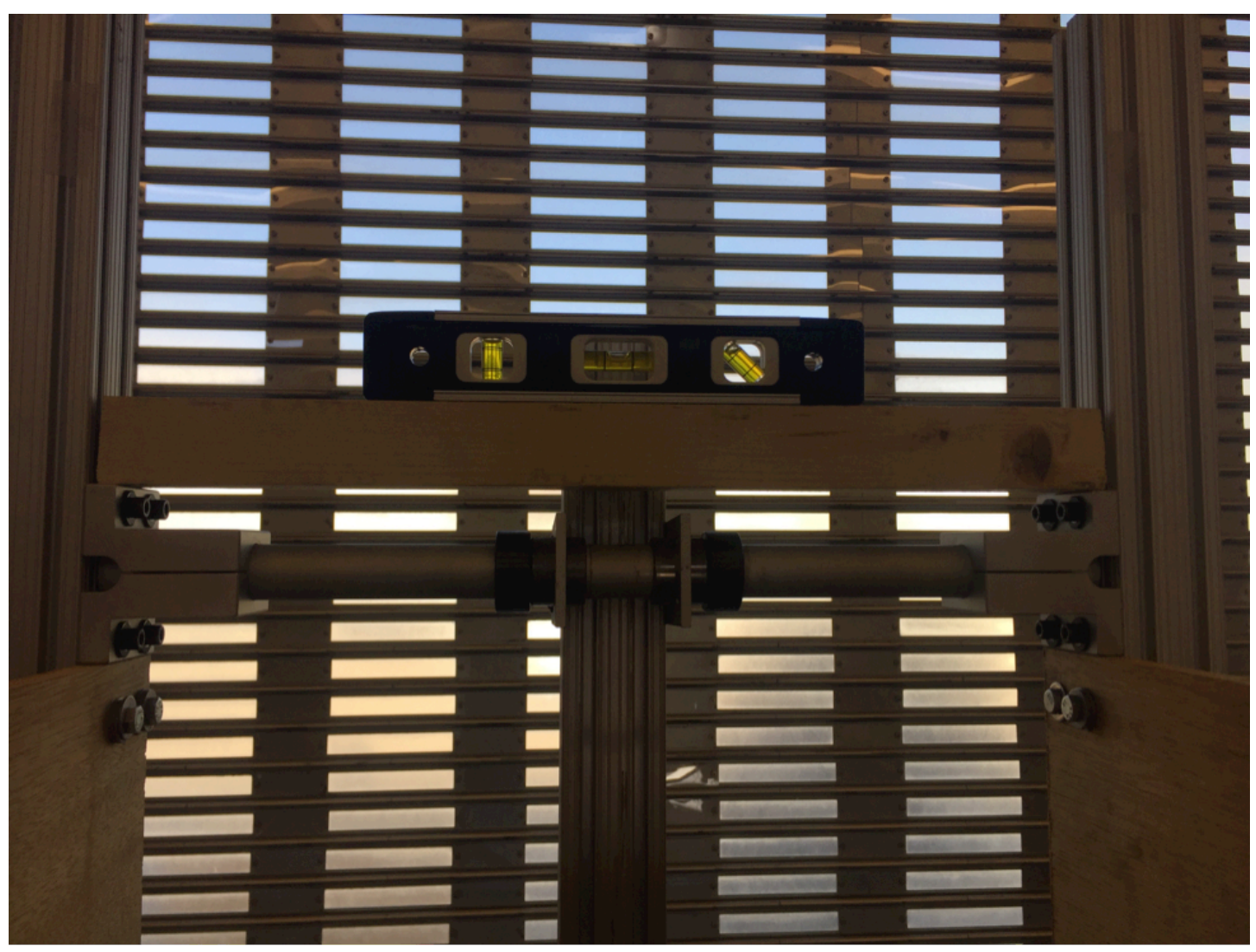

Finish by sliding the repeatability plates all the way up to the brackets as shown above and tightening the bolts. 


\section{A.3 Running and Analyzing a Test}

At this point the "Start" button should be clicked and the program should be observing the data, waiting for a shock event.

1) Make sure that everyone in the vicinity knows that testing is happening, as the impacts are loud. Anyone in the room should have ear protection, and anyone near the shock table should have eye protection (especially if there is something mounted to the table).

2) Pull the hammer arm back until it touches the bar in the repeatability plates.

3) Release the arm.

4) After impact, catch the hammer arm before it impacts the table a second time.

5) Click "Stop" and save the data as discussed previously.

6) Open and run Matlab file "Cal Poly SRS".

7) The default values for frequency scheme, start and end frequencies, and damping ratio are all industry standard and can likely be left alone. "Accel of interest" allows the user to choose which accelerometer's data to plot. The options are 0-3. 0 indicates that all three channels should be plotted, while 1 corresponds to channel 1 (accelerometer 8158 if the wiring suggestions were followed), 2 corresponds to channel 2, and 3 corresponds to channel 3.

8) Check the plots for zero shift. The raw acceleration data not returning to a zero baseline after the shock event, the velocity data quickly diverging from zero, and the positive and negative SRS curves being drastically different under $1000 \mathrm{~Hz}$ all indicate zero shift. 
9) If there are no signs of zero shift, the data is good and the SRS can be checked for acceptance. 


\section{B CAD}

\section{B.1 Hammer Heads}

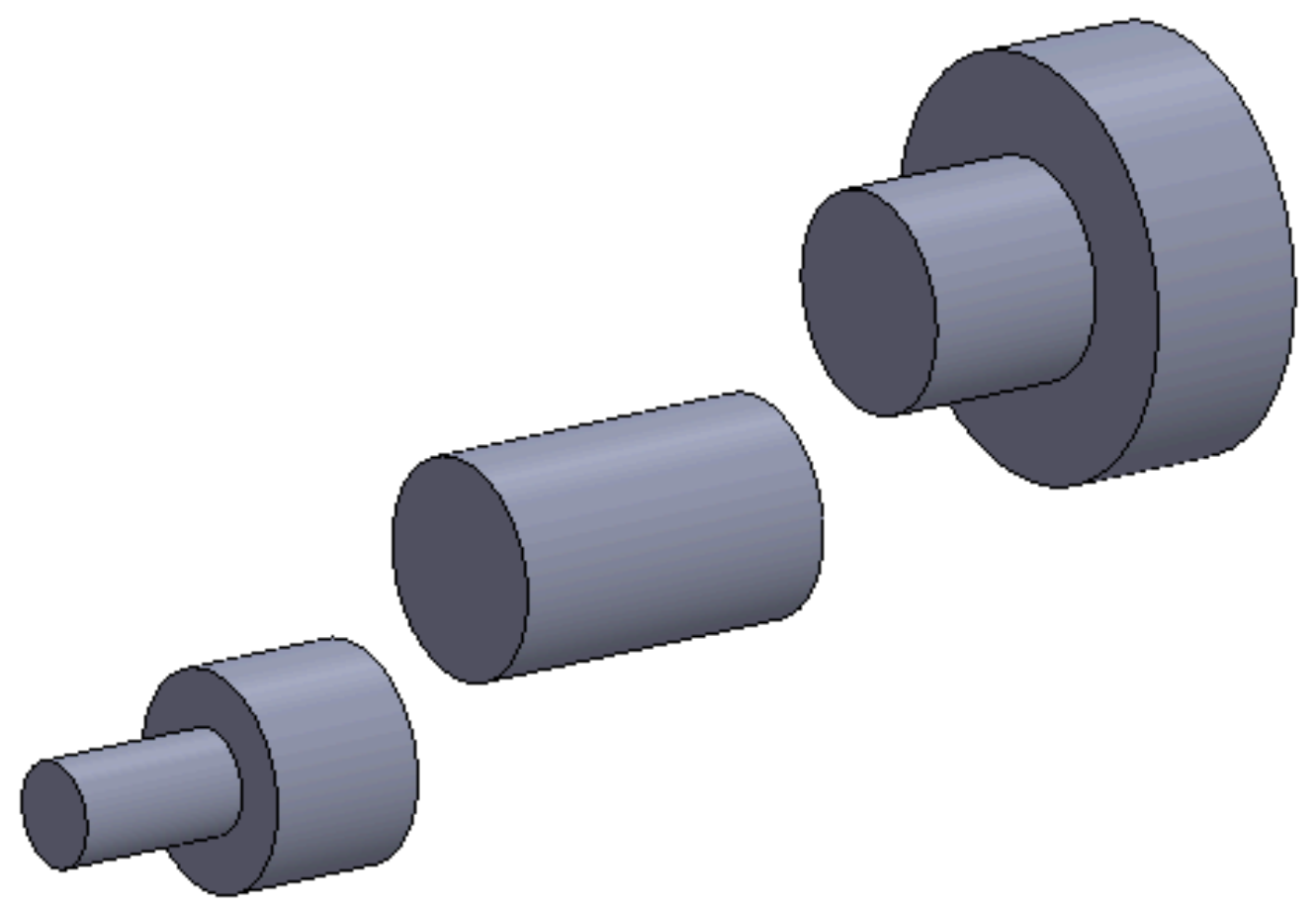




\section{B.2 New Hammer}

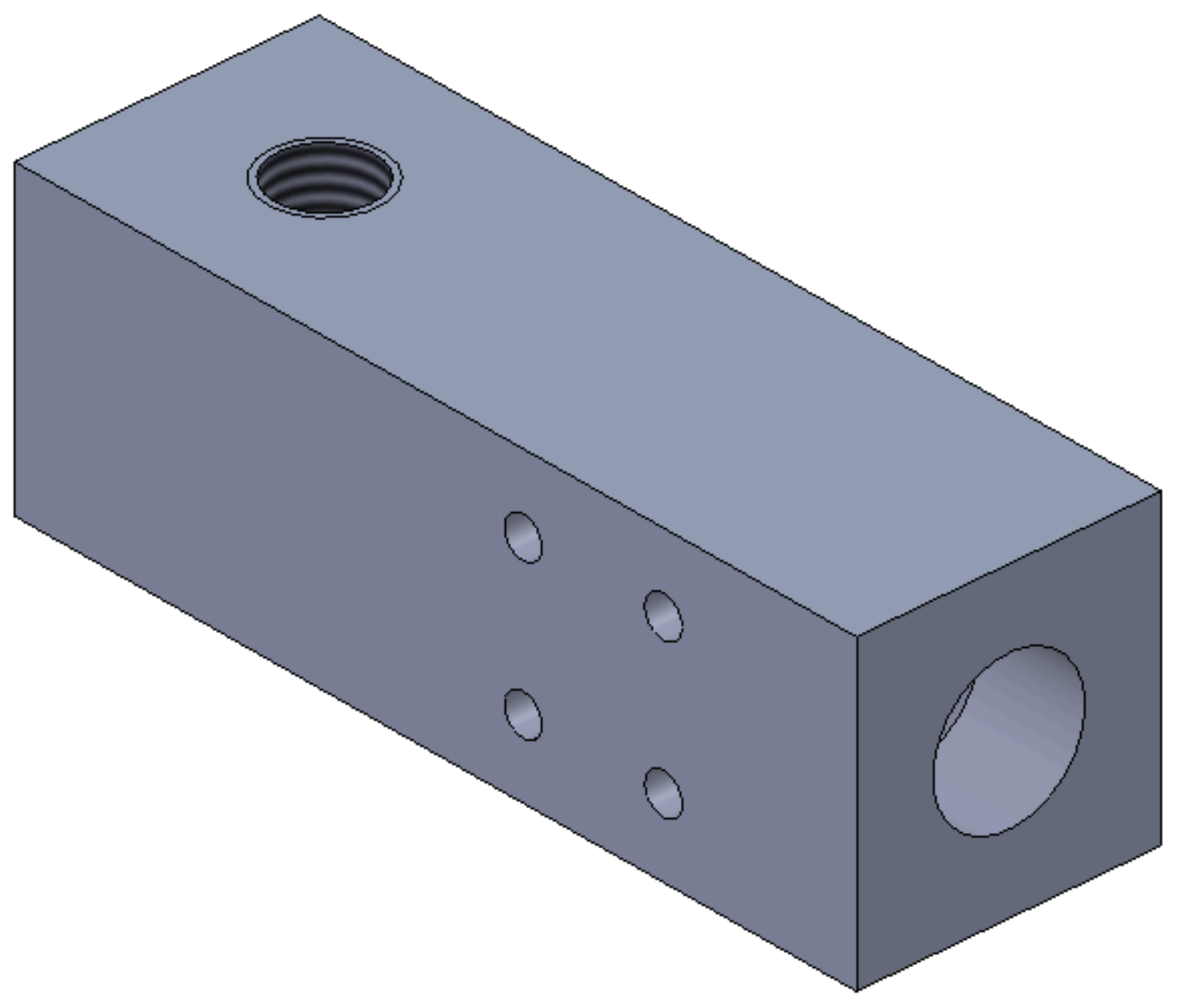




\section{B.3 Repeatability Plates}

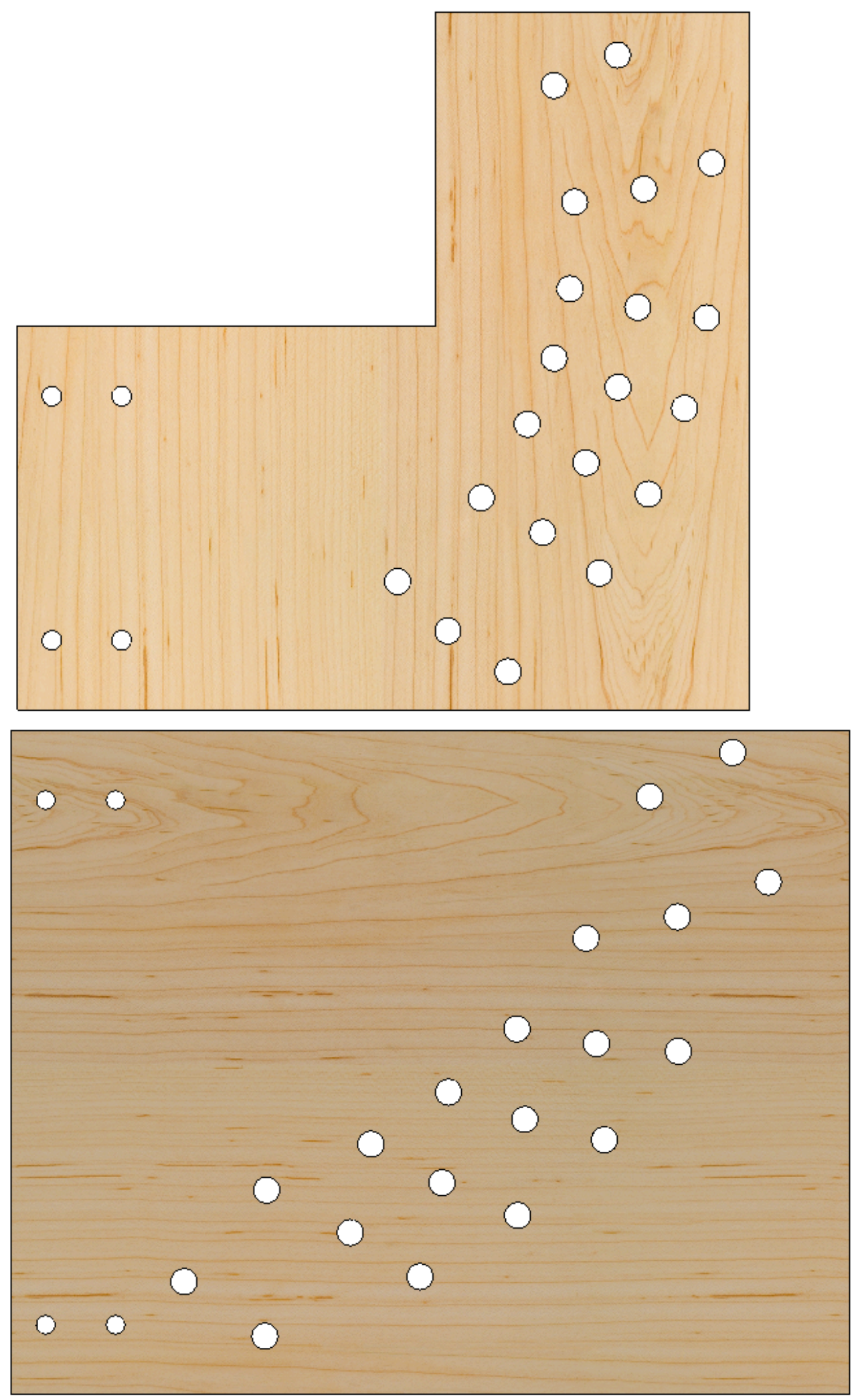




\section{B.4 L-Shaped Interface Fixture}

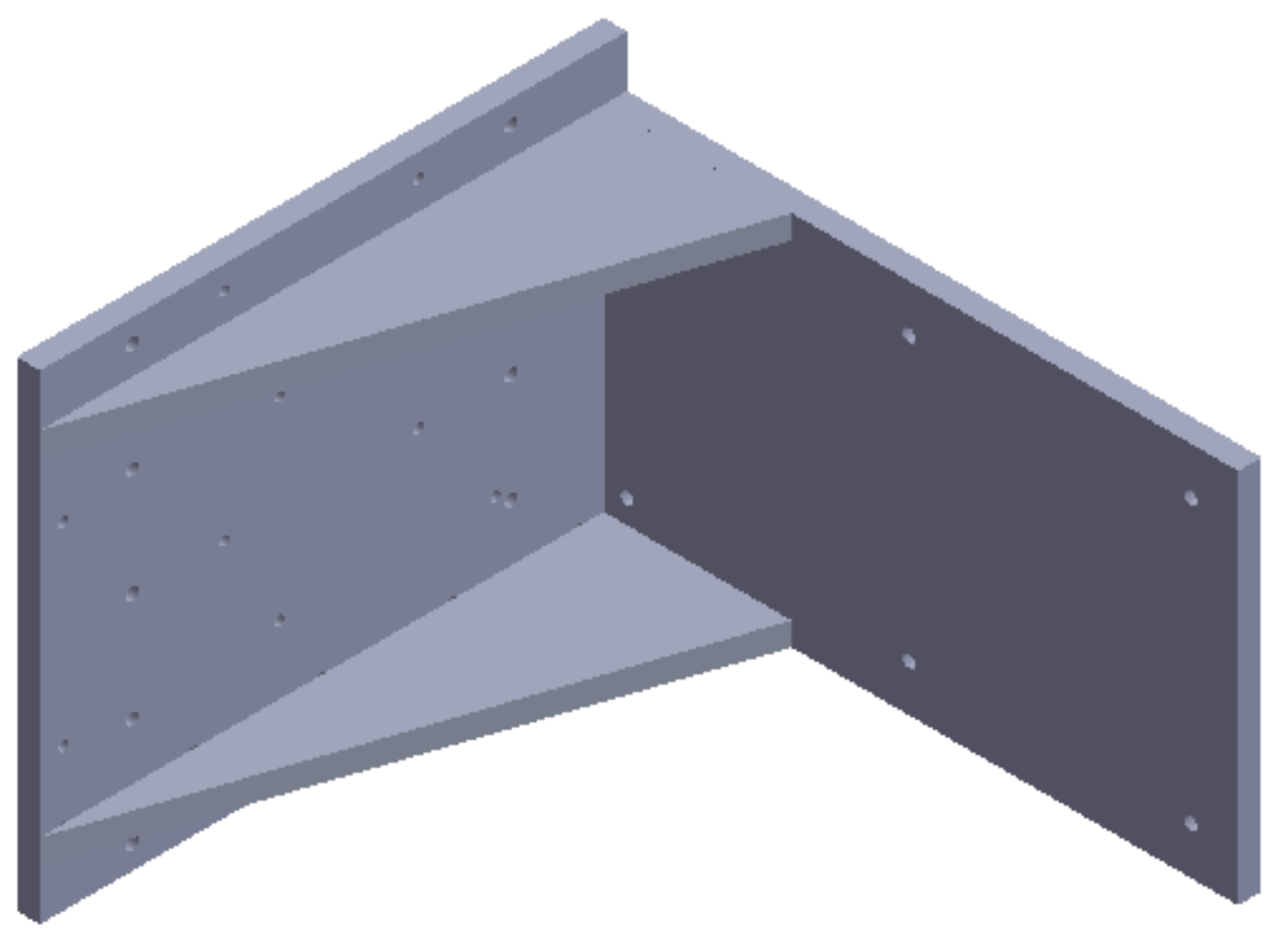

